\title{
Spiders (Arachnida: Aranei) of Abkhazia
}

\author{
Пауки (Arachnida: Aranei) Абхазии
}

\author{
Mykola M. Kovblyuk' ${ }^{1}$, Yuri M. Marusilk ${ }^{2}$ Aleksandr V. Ponomarev ${ }^{3}$, \\ Valery A. Gnelitsa ${ }^{4}$, Anton A. Nadolny ${ }^{1}$ \\ Н.М. Ковбцюк ${ }^{1}$ Ю.М. Марусик ${ }^{2}$, А.В. Пономарёв ${ }^{3}$, \\ В.А. Гнелища ${ }^{4}$, А.А. Надольный ${ }^{1}$
}

\footnotetext{
${ }^{1}$ Zoology Department, National Taurida V.I. Vernadsky University, Yaltinskaya street 4, Simferopol 95007, Ukraine. E-mail: kovblyuk@mail.ru; nadolnyanton@mail.ru

${ }^{2}$ Institute for Biological Problems of the North, RAS, Portovaya Str. 18, Magadan 685000, Russia. E-mail: yurmar@mail.ru

${ }^{3}$ Institute of Arid Zones, South Scientific Centre RAS, Chekhov str., 41, Rostov-on-Don 344006 Russia. E-mail: ponomarev1952@mail.ru

${ }^{4}$ Biological Faculty, National Taras Shevchenko University of Kiev, Volodymyrska street 64, Kiev 01033, Ukraine. E-mail: gnelitsa@mail.ru

${ }^{1}$ Кафедра зоологии Таврического национального университета им. В.И.Вернадского, ул. Ялтинская 4, Симферополь 95007, Украина.

${ }^{2}$ Институт Биологических Проблем Севера ДВО РАН, ул. Портовая 18, Магадан 685000, Россия.

${ }^{3}$ Институт аридных зон ЮНЦ РАН, пр. Чехова, 41, Ростов-на-Дону 344006 Россия.

${ }^{4}$ Биологический факультет Киевского национального университета им. Т.Г. Шевченко, ул. Владимирская 64, Киев 01033, Украина.
}

KEY WORDS: spiders, Aranei, annotated check-list, West Caucasus, Abkhazia.

КЛЮЧЕВЫЕ СЛОВА: пауки, аннотированный список, Западный Кавказ, Абхазия.

ABSTRACT: A list of 283 species belonging to 42 families found in Abkhazia is presented. Eleven families (Atypidae, Coelotidae, Dictynidae, Filistatidae, Hahniidae, Leptonetidae, Liocranidae, Mysmenidae, Philodromidae, Theridiosomatidae, Zoridae) and 130 species have been found in Abkhazia for the first time. Seven species are illustrated.

РЕЗЮМЕ: Приведён список пауков, выявленных в Абхазии (по литературным и собственным данным): 283 вида из 42 семейств. Из них 11 семейств (Atypidae, Coelotidae, Dictynidae, Filistatidae, Hahniidae, Leptonetidae, Liocranidae, Mysmenidae, Philodromidae, Theridiosomatidae, Zoridae) и 130 видов указываются для Абхазии впервые. Семь видов иллюстрированы.

\section{Introduction}

Abkhazia is the small region on the southwestern edge of the Caucasus Major, at the northeastern shore of the Black Sea and south of Russia. Spiders of this area were never studied purposely and therefore the fauna of Abkhazia remains rather poorly known . First spiders from this region were reported by Schmidt [1895], Simon [1899], Verzhbitsky [1902] and Charitonov [1939, 1941, 1947]. In last papers Charitonov described four new species from the caves (Tegenaria abchasica Charitonov, 1941; Cybaeus abchasicus
Charitonov, 1947; Carpathonesticus caucasicus (Charitonov, 1947) and C. zaitzevi (Charitonov, 1939)). Further contributions were made by Buchar [1966], Mcheidze \& Utotchkin [1971], Tanasevitch [1986, 1990] and Dunin [1992a,b]. They described seven species from Abkhazia (Pirata hurkai Buchar, 1966; Xysticus kochi abchasicus Mcheidze et Utotchkin, 1971; Hygrocrates caucasicus Dunin, 1992; Diplocephalus caucasicus Tanasevitch, 1987; Oedothorax meridionalis Tanasevitch, 1987; Stemonyphantes agnatus Tanasevitch, 1990 and Troglohyphantes deelemanae Tanasevitch, 1986). Further contribution was made by Mcheidze [1997] who surveyed fauna of Georgia (including Abkhazia). Recently, during the last decade, Abkhazia and its spider fauna became a subject of intensive studies and seven papers dealing with different groups of spiders have been published [Marusik et al., 2004; Marusik \& Kovblyuk, 2004; Kovblyuk \& Ponomarev, 2008; Kovblyuk \& Nadolny, 2009; Marusik \& Gnelitsa, 2009; Marusik \& Kovblyuk, 2010; Kovblyuk et al., 2010]. This became possible thanks to several expeditions held by Russian and Ukrainian arachnologists in 2003, 2004, 2008 and 2009. All these data were summarized in the database of Caucasian spiders [Otto \& Dietzold, 2006]. So, until recently 153 species of spiders were known in Abkhasia.

Most of the material collected by two first authors in Abkhazia was identified, but a list of these identifications was never published. It is the main aim of this paper to summarize all literature and collection data 
concerning spiders of Abkhazia and to present the first checklist of spiders from the region.

\section{Material and Methods}

Most of new material presented here was collected by two first authors, and also by our colleagues Yu.G. Arzanov, P.P. Ivliev, E.A. Khachikov and N.N. Yunakov in the period 2001-2010.

Besides the identified species, we also list several unidentified ones, in case they belong to interesting supraspecific taxa. Numbers of identified and unidentified species are presented in table 1. Several species represented in our collection by juvenile specimens were not included in the list, although they can be identified with high certainty because of a characteristic body shape or pattern.

Numbers of samples are provided in the text in square brackets in bold. Detailed data for all 63 localities visited and surveyed are indicated on Map 1 as follows (in chronological order):

01. Gudauta Distr., Bzyb Mt. Range, Achybakh Mt., 30.06.2001, Yu.G. Arzanov;

02. Gudauta Distr., confluence of Bzyb and Gega Rivers, 6.07.2001, Yu.G. Arzanov;

03. Gagra, 12.07.2001, Yu.G. Arzanov;

04. Gudauta Distr., Khabyu Vil., 4-7.06.2003, P.P. Ivliev;

05. Gudauta Distr., Khabyu Vil., 22-24.06.2003, P.P. Ivliev;

06. Sukhum, University Campus $\left(42^{\circ} 58.4^{\prime} \mathrm{N} 41^{\circ}\right.$ 04.04' E, ca $50 \mathrm{~m}$ ), litter, Eucalyptus, tree shaking, 7.12.2003, Yu.M. Marusik;

07. Sukhum, Botanical Garden $\left(43^{\circ} 00.329^{\prime} \mathrm{N}\right.$ $\left.41^{\circ} 01.400^{\prime} \mathrm{E}\right)$, litter and tree shaking, 9.12.2003, Yu.M. Marusik \& G.N.Antipova;

08. Sukhum, Kelasur $\left(42^{\circ} 58.4^{\prime} \mathrm{N} 41^{\circ} 04.04^{\prime} \mathrm{E}\right)$, mainly under Eucalyptus bark, 9-10.12.2003, Yu.M. Marusik;

09. Gudauta Distr., $25 \mathrm{~km} \mathrm{~N}$ Gudauta, $15 \mathrm{~km} \mathrm{~N}$ from Achandara Vil., Gunarkhva, 12-14.08.2004, P.P. Ivliev;

10. Sukhum ( $\left.42^{\circ} 58.4^{\prime} \mathrm{N} 41^{\circ} 04.04^{\prime} \mathrm{E}\right), 9-10.10 .2004$, Yu.M. Marusik;

11. Sukhum $\left(42^{\circ} 58.4^{\prime} \mathrm{N} 41^{\circ} 04.04^{\prime} \mathrm{E}\right)$, sandy seashore, on grass, 9-10.10.2004, Yu.M. Marusik;

12. Sukhum $\left(42^{\circ} 58.4^{\prime} \mathrm{N} 41^{\circ} 04.04^{\prime} \mathrm{E}\right)$, litter of coniferous trees along the seashore, 10.10.2004, Yu.M. Marusik;

13. Sukhum, shaking trees, 10.10.2004, Yu.M. Marusik;

14. Sukhum, left bank of Kelasur River $\left(42^{\circ} 58^{\prime} \mathrm{N}\right.$ 41 $04^{\prime}$ 'E), clay shadowed cliffs, 11.10.2004, Yu.M. Marusik;

15. Sukhum, left bank of Kelasur River $\left(42^{\circ} 58^{\prime} \mathrm{N}\right.$ $\left.41^{\circ} 04^{\prime} \mathrm{E}\right)$, cellar of uninhabited house, 11.10.2004, Yu.M. Marusik;

16. Sukhum, left bank of Kelasur River, tree shaking in university campus, 11.10.2004, Yu.M. Marusik;
17. Sukhum, left bank of Kelasur River $\left(42^{\circ} 58^{\prime} \mathrm{N}\right.$ $\left.41^{\circ} 04^{\prime} \mathrm{E}\right)$, litter along river bank, 11.10.2004, Yu.M. Marusik;

18. Sukhum, Botanical Garden, 13.10.2004, Yu.M. Marusik;

19. Gudauta Distr., road to Ritsa Lake $\left(43^{\circ} 28^{\prime} \mathrm{N}\right.$, $40^{\circ} 30^{\prime} \mathrm{E}$ ), shaking moss epiphytes and in moss, 14.10. 2004, Yu.M. Marusik;

20. Gudauta Distr., road to Ritsa Lake $\left(43^{\circ} 15^{\prime} \mathrm{N}\right.$ $\left.40^{\circ} 20^{\prime} \mathrm{E}\right)$, gravels on Bzyb’ River bank, 14.10.2004, Yu.M. Marusik;

21. Gudauta Distr., road to Ritsa Lake $\left(43^{\circ} 28^{\prime} \mathrm{N}\right.$ $\left.40^{\circ} 30^{\prime} \mathrm{E}\right)$, shaking moss epiphytes and in moss, 14.10. 2004, Yu.M.Marusik;

22. Gagra Distr., env. of Pitsunda, Ldzaa Vil., Myusser highland $\left(43^{\circ} 10.756^{\prime} \mathrm{N} 40^{\circ} 21.244^{\prime} \mathrm{E}\right)$, Pinus pityusa litter and branch shaking on steep slope, 15.10.2004, A. Shavlokhov;

23. Gagra Distr., env. of Pitsunda, Ldzaa Vil., My-

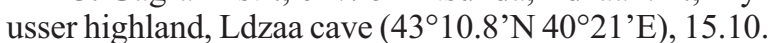
2004, Yu.M. Marusik \& A. Shavlokhov;

24. Gagra Distr., env. of Pitsunda, Ldzaa Vil., Myusser highland $\left(43^{\circ} 10.756^{\prime} \mathrm{N} 40^{\circ} 21.244^{\prime} \mathrm{E}\right)$, Pinus pityusa litter on steep slope, 15.10.2004, Yu.M. Marusik;

25. Gagra Distr., env. of Pitsunda, Ldzaa Vil., Myusser highland $\left(43^{\circ} 10.756^{\prime} \mathrm{N} 40^{\circ} 21.244^{\prime} \mathrm{E}\right), 15-16.10$. 2004, Yu.M. Marusik;

26. Gagra Distr., env. of Pitsunda, Ldzaa Vil., Myusser highland $\left(43^{\circ} 10.255^{\prime} \mathrm{N} 40^{\circ} 22.370^{\prime} \mathrm{E}\right)$, full grown Pinus pityusa along seashore, litter and shaking, 16.10. 2004, A. Shavlokhov;

27. Gagra Distr., env. of Pitsunda, Ldzaa Vil., Myusser highland $\left(43^{\circ} 10.255^{\prime} \mathrm{N} 40^{\circ} 22.370^{\prime} \mathrm{E}\right)$, full grown Pinus pityusa along seashore, litter, 16.10.2004, Yu.M. Marusik;

28. Gagra Distr., env. of Pitsunda, Ldzaa Vil., Myusser highland $\left(43^{\circ} 10.255^{\prime} \mathrm{N} 40^{\circ} 22.370^{\prime} \mathrm{E}\right)$, young $2-$ $3 \mathrm{~m}$ Pinus pityusa along seashore, litter, 16.10.2004, Yu.M. Marusik;

29. Gagra Distr., Bzyb Vil., 13-15.06.2005, P.P. Ivliev;

30. Gudauta, 7.10.2005, E.A. Khachikov;

31. Sukhhum Distr., West Gumista River, middle

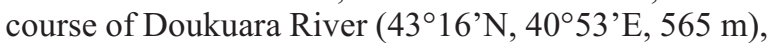
22.06.2007, N.N. Yunakov;

32. Gagra Distr., middle course of Ghegha River $\left(43^{\circ} 25^{\prime} \mathrm{N}, 40^{\circ} 26^{\prime} \mathrm{E}, 500 \mathrm{~m}\right), 12.07 .2007$, N.N. Yunakov;

33. Gudauta Distr., $25 \mathrm{~km} \mathrm{~N}$ Gudauta, $15 \mathrm{~km} \mathrm{~N}$ from Achandara Vil., Gunarkhva, 7-10.10.2007, P.P. Ivliev;

34. Gudauta Distr., Malaya Ritsa Lake, 6.06.2008, P.P. Ivliev;

35. Gagra Distr., Ritsa Lake, 7.06.2008, P.P. Ivliev;

36. Gudauta Distr., environs of Pitsunda, mouth of Ryapsh River, Myusser Distr. of Pitsundo-Myusser Reserve $\left(43^{\circ} 09^{\prime} \mathrm{N}, 40^{\circ} 25^{\prime} \mathrm{E}, 27 \mathrm{~m}\right)$, kolhida wood (Arbutus, Alnus, Ficus, Carpinus, Quercus, Acer, Rhododendron, Erica arborea, Rubus, Smilax excelsa, Castanea sativa, Laurocerasus officinalis, Cornus mas, Pinus pityusa, Crataegus, Tilia, Ruscus), 1-7.07.2008, M.M. Kovblyuk; 


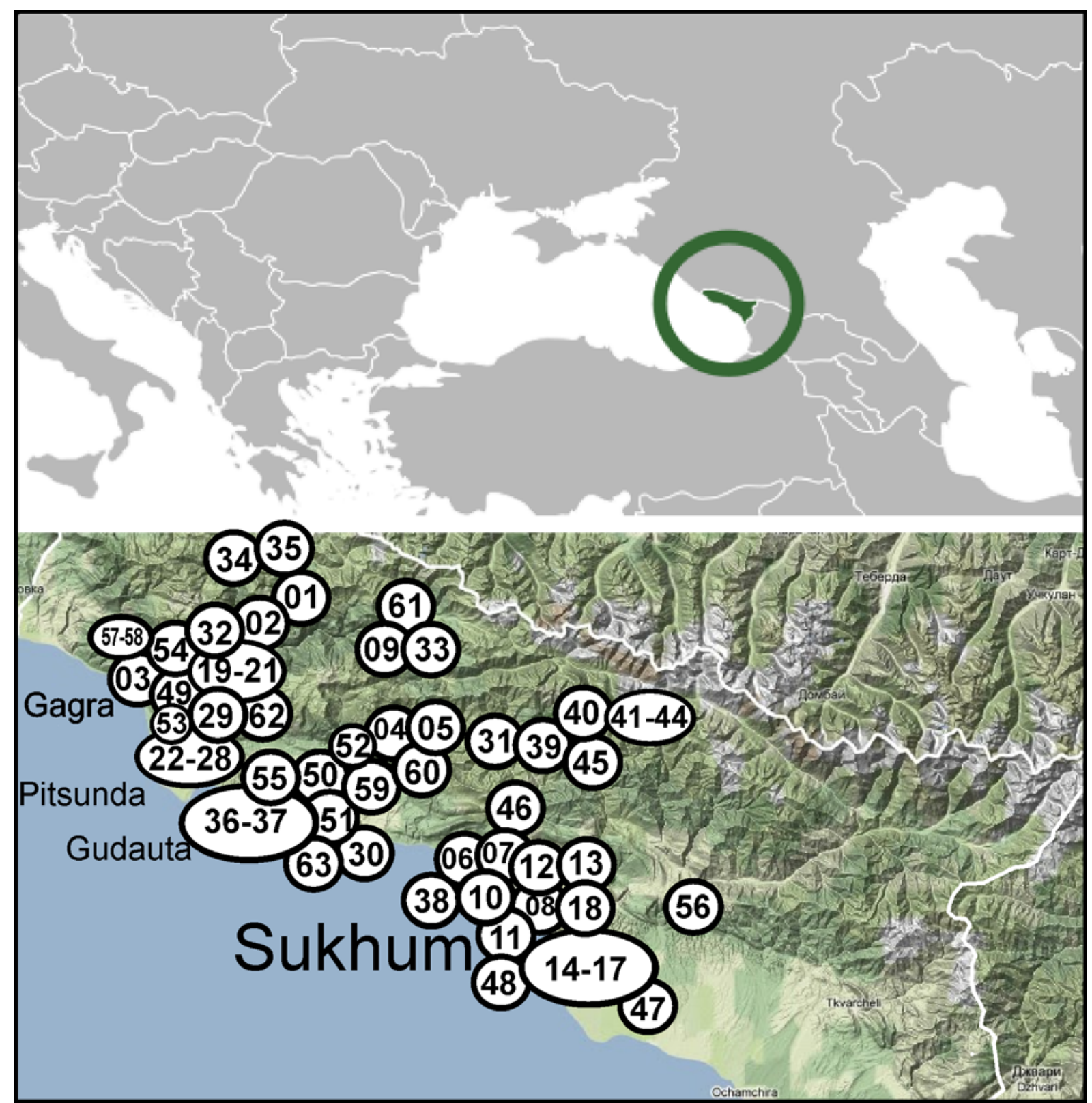

Map 1. Collecting sites in Abkhazia.

Карта 1. Места сбора материала в Абхазии.

37. Gudauta Distr., environs of Pitsunda, Myusser Distr. of Pitsundo-Myusser Reserve $\left(43^{\circ} 09^{\prime} \mathrm{N}, 40^{\circ}\right.$ 25'E), cave, 6.07.2008, N.N. Yunakov;

38. Sukhum, 8.07.2008, M.M. Kovblyuk;

39. Sukhum Distr., Gumysta Reserve, East Gumysta River, kordon Tsymur $\left(43^{\circ} 10^{\prime} \mathrm{N}, 41^{\circ} 02^{\prime} \mathrm{E}, 420 \mathrm{~m}\right.$, wood with Fagus, Acer and Castanea sativa, 8-16.07. 2008, M.M. Kovblyuk;

40. Sukhum Distr., Gumista Reserve, West slope of Dzykhva Mt. $\left(43^{\circ} 12^{\prime} \mathrm{N}, 41^{\circ} 05^{\prime} \mathrm{E}, \sim 650 \mathrm{~m}\right)$, forest and grassland, 17-19.07.2008, M.M. Kovblyuk;

41. Sukhum Distr., Buru Range, Dzykhva Mt., KotKot River $\left(43^{\circ} 13^{\prime} \mathrm{N}, 41^{\circ} 07^{\prime} \mathrm{E}, \sim 2300 \mathrm{~m}\right)$, alpine zone, 19-26.07.2008, M.M. Kovblyuk;
42. Sukhum Distr., Buru Range, Dzykhva Mt., KotKot River ( $43^{\circ} 13^{\prime} \mathrm{N}, 41^{\circ} 07^{\prime} \mathrm{E}, \sim 2500 \mathrm{~m}$ ), alpine zone, near of snow, 24.07.2008, A.A. Khaustov;

43. Sukhum Distr., Buru Range, Dzykhva Mt.,

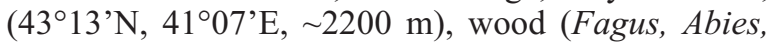
Acer), 25.07.2008, M.M. Kovblyuk;

44. Sukhum Distr., Buru Range, Dzykhva Mt., West slope, wood, 26.07.2008, M.M. Kovblyuk;

45. Sukhum Distr., Gumysta Reserve, East Gumysta River, kordon Tsymur $\left(43^{\circ} 10^{\prime} \mathrm{N}, 41^{\circ} 02^{\prime} \mathrm{E}, 420 \mathrm{~m}\right.$, wood with Fagus, Acer and Castanea sativa, 26-29.07. 2008, M.M. Kovblyuk;

46. Sukhum Distr., Zemo-Birtskha Vil., 30.07.2008, M.M. Kovblyuk; 
47. Gulripsh Distr., Kelasur River, Bagramany, Sharvashidaevsky Forest $\left(42^{\circ} 58^{\prime} \mathrm{N}, 41^{\circ} 04^{\prime} \mathrm{E}, 146 \mathrm{~m}\right), 31.07$. 2008, M.M. Kovblyuk \& N.N. Yunakov;

48. Sukhum, 30-31.07.2008, M.M. Kovblyuk;

49. Gagra Distr., Bzyb Vil., forest, 4.11.2008, P.P. Ivliev;

50. Gudauta Distr., Achandara Vil., 5.11.2008, P.P. Ivliev;

51. Gudauta, 6.11.2008, P.P. Ivliev;

52. Gudauta Distr., Achandara Vil., 1-4.05.2009, P.P. Ivliev;

53. Gagra Distr., Bzyb Vil., 4-10.05.2009, P.P. Ivliev;

54. Gagra Distr., Gagra Range, Mamdzyshkha Mt. $(1866 \mathrm{~m})$, from border of forest $\left(43^{\circ} 18^{\prime} \mathrm{N}, 40^{\circ} 19^{\prime} \mathrm{E}\right.$, $1705 \mathrm{~m}$ ) to peak, wood (Abies, Fagus, Acer) and alpine meadows, 7-15.07.2009, M.M. Kovblyuk;

55. Gudauta Distr., environs of Pitsunda, Myusser Distr. of Pitsundo-Myusser Reserve, left bank of Ryapsh River $\left(43^{\circ} 10^{\prime} \mathrm{N}, 40^{\circ} 25^{\prime} \mathrm{E}, 23 \mathrm{~m}\right)$, kolkhida wood, sea and river coast, humid gorges, 15-24.07.2009, M.M. Kovblyuk \& N.N. Yunakov;

56. Ochamchyra Distr., left bank of Kodor River, foothills of Kodor Mt. Range, environs of Atara-Armyanskaya Vill. and Naa Vill. (42 $53^{\prime}-57^{\prime} \mathrm{N}, 41^{\circ} 17^{\prime}-$ 19'E, 141-253 m), wood with Alnus, Quercus, Fagus, Castanea sativa, Carpinus, Aesculus, Corylus, Tilia and Rhododendron, glades, 24.07-04.08.2009, M.M. Kovblyuk \& N.N. Yunakov;

57. Gagra Distr., Gagra Mt. Range, forest $\left(43^{\circ} 15^{\prime} \mathrm{N}\right.$, 40²2’E, 825 m), 15.07.2009, N.N. Yunakov;

58. Gagra, forest, 15.07.2009, M.M. Kovblyuk;

59. Gudauta Distr., Achandara Vil., 1-2.07.2010, P.P. Ivliev; Ivliev;

60. Gudauta Distr., Khabyu Vil., 1.07.2010, P.P.

61. Gudauta Distr., $25 \mathrm{~km} \mathrm{~N}$ Gudauta, $15 \mathrm{~km} \mathrm{~N}$ from Achandara Vil., Gunarkhva, 3-5.07.2010, P.P. Ivliev;

62. Gagra Distr., Bzyb, 9.07.2010, P.P. Ivliev;

63. Gudauta, 10.07.2010, P.P. Ivliev.

All specimens were deposited in the following collections:

CP - private collection of A.V. Ponomarev, Rostov-on-Don, Russia;

TNU - Zoology Department, V.I. Vernadsky Taurida National University, Simferopol, Ukraine, M.M. Kovblyuk;

VAG - private collection of V.A. Gnelitsa, Sumy, Ukraine;

YMC - temporary collection of Yu.M. Marusik, Zoological Museum, University of Turku, Finland;

ZMMU - Zoological Museum of the Moscow State University, Moscow, Russia, K.G. Mikhailov.

Doubtful and not confirmed records are marked by “?”, and misidentifications by “??”. Species described from Abkhazia are marked by asterisk (*).
Distribution range names were given mainly after the system proposed by K.B. Gorodkov [1984] and used in recent works [cf. Marusik et al., 2000]. The term "Ancient Mediterranean" sensu Kryzhanovsky [2002] refers to the region stretching from Macaronesia, across whole Mediterranean, Middle Asia to Gobi Desert and Tibet (ca to $97^{\circ} \mathrm{E}$ ). This region coincides with limits of the ancient Tethys Sea.

All scale bars are equal to $0.1 \mathrm{~mm}$.

\section{Survey of species}

AGELENIDAE (3 genera and 7 species)

Agelena labyrinthica (Clerck, 1757)

RECORDS. Sukhum [Spassky, 1937].

MATERIAL. 1 O $^{T}$ (TNU-2644/15) [45]; 3 우 (TNU-2645/1) [46]; $1 O^{\top}, 1$ (TNU-2653/26) [56]; 1 ( (CP) [60].

DISTRIBUTION. Trans-Palaearctic nemoral range: from Portugal and Spain east to Maritime Province of Russia, Korea and Japan; and from Scandinavia south to Sardinia, Sicily, Crete and China [Mikhailov, 1997; Marusik et al., 2000; Helsdingen, 2010; Platnick, 2010].

\section{Tegenaria abchasica Charitonov, 1941*}

RECORDS. Sukhum Distr.: Kelasur [Charitonov, 1941, 1947; Dzhanashvilli, 1965; Mcheidze, 1964, 1997; Otto \& Dietzold, 2006].

MATERIAL. 1 Oొ $^{7}$ YMC) [17]; 2 ㅇ (TNU-2636/44) [36]; 2 $\sigma^{7} \sigma^{7}$ (TNU-2641/13) [41]; $1 O^{7}(\mathrm{CP}),[\mathbf{5 2}]$.

DISTRIBUTION. Caucasus: Krasnodar Area of Russia, Abkhazia, Georgia [Kovblyuk \& Ponomarev, 2008]. 2008

Tegenaria chumachenkoi Kovblyuk \& Ponomarev,

MATERIAL. 3 우 (TNU-2652/4) [54]; 3 우 (CP) [53].

DISTRIBUTION. Caucasus: Krasnodar and Stavropol Areas of Russia [Kovblyuk \& Ponomarev, 2008]; recorded from Abkhazia for the first time.

Tegenaria longimana Simon, 1898

MATERIAL. $1 O^{7}$ (TNU-2636/43) [36]; $1 O^{7}$ (CP) [53].

DISTRIBUTION. Caucasian coast of Black Sea: Krasnodar Area of Russia, Abkhazia (first record), Georgia, northeastern Turkey [Kovblyuk \& Ponomarev, 2008].

\section{Tegenaria parietina (Fourcroy, 1785)}

RECORDS. Akhali Atoni, Sukhum [Mcheidze, 1964, 1997; Otto \& Dietzold, 2006].

DISTRIBUTION. Mediterranean and Caucasus, and Uruguay and Argentina (probably introduced), North Africa, Europe, Caucasus: from Portugal east to Azerbaijan; and from Britain and Danish mainland south to North Africa [Mikhailov, 1997; Helsdingen, 2010; Platnick, 2010]. 
Tegenaria taurica Charitonov, 1947

RECORDS. Akhali Atoni [Mcheidze, 1997; Otto \& Dietzold, 2006].

DISTRIBUTION. Crimea, Abkhazia and Georgia [Mcheidze, 1997; Esyunin \& Farzalieva, 2002; Kovblyuk, 2004a; personal data].

\section{Tegenaria spp.}

MATERIAL. 5 우 (TNU-2636/45) [36]; 1 (TNU-2638/9) [38]; 4 우 (TNU-2639/7) [39]; 3 우 (TNU-2640/9) [40]; 1 क (TNU-2642/3) [43]; 2 우 (TNU-2652/5) [54]; 5 우 (TNU-2651/9) [55]; 1 ㅇ (TNU-2651/33) [55]; 5 우 (TNU-2653/27) [56].

NOTE. Several Tegenaria females of unclear taxonomic position were collected in expeditions of 20082009. Their identifications are problematic without males.

Textrix denticulata (Olivier, 1789)

MATERIAL. 2 우 (TNU-2636/42) [36]; $4 \bigcirc^{\top} \bigcirc^{7}, 2$ 우 (TNU2651/8) [55].

DISTRIBUTION. European-Caucasian nemoral range: from Portugal east to Urals, and from Norwegian mainland south to Sardinia [Mikhailov, 1997; Helsdingen, 2010; Platnick, 2010]. Species is recorded from Caucasus for the first time.

AMAUROBIIDAE (1 genus and 1 species)

Amaurobius antipovae Marusik \& Kovblyuk, 2004*

RECORDS. Sukhum, Kelasur [Marusik \& Kovblyuk, 2004; Otto \& Dietzold, 2006].

MATERIAL. 1 (CP) [49]; 1 ( $(\mathrm{CP})[53]$.

DISTRIBUTION. Caucasus: Adygeya and Krasnodar Area (Russia) and Abkhazia [Marusik \& Kovblyuk, 2005; Ponomarev \& Chumachenko, 2007; Ponomarev, 2009].

ANYPHAENIDAE (1 genus and 1 species)

Anyphaena accentuata (Walckenaer, 1802)

RECORDS. Sukhum [Spassky, 1937; Mcheidze, 1997; Otto \& Dietzold, 2006].

MATERIAL. 1 + (YMC) [13]; $20^{7} \sigma^{7}, 1$ \& (CP) [53].

DISTRIBUTION. West Palaearctic nemoral range, eastern limit is Kopetdagh Mt. range [Mikhailov, 1997; Helsdingen, 2010; Platnick, 2010].

ARANEIDAE (16 genera and 22 species)

Aculepeira ceropegia (Walckenaer, 1802)

MATERIAL. 2 우 (TNU-2641/5) [41]; $20^{\top} \sigma^{7}, 8$ 우 (TNU2652/19) [54].

DISTRIBUTION. West Palaearctic boreo-nemoral range: Europe east to Caucasus; from Norwegian mainland to Sardinia and Sicily [Mikhailov, 1997; Helsdingen, 2010; Platnick, 2010].
Agalenatea redii (Scopoli, 1763)

RECORDS. Sukhum [Mcheidze, 1997 — sub Araneus; Otto \& Dietzold, 2006].

DISTRIBUTION. West-Central Palaearctic nemoral range: from Europe to Xinjiang [Mikhailov, 1997; Helsdingen, 2010; Platnick, 2010].

Araneus angulatus Clerck, 1757

MATERIAL. $1 O^{\top}, 2$ 우 (CP-27.10.8/7) [03]; $1 O^{7}$ (TNU-2636/ 10) $[36]$.

DISTRIBUTION. West-Palaearctic polyzonal range (absent in Siberia) [Mikhailov, 1997; Helsdingen, 2010; Platnick, 2010].

Araneus diadematus Clerck, 1757

RECORDS. Akhali Atoni, Gagra, Gudauta, Sukhum [Spassky, 1937; Mcheidze, 1964, 1997; Otto \& Dietzold, 2006].

MATERIAL. $1 \sigma^{7}$ (YMC) [19]; 1 (YMC) [26]; 1 (ZMMU) [33]; 1 + (TNU-2641/6) [41]; 1 \& (CP-27.10.2/18) [49].

DISTRIBUTION. Circum-Holarctic nemoral range [Mikhailov, 1997; Helsdingen, 2010; Platnick, 2010].

Araneus marmoreus Clerck, 1757

MATERIAL. $1 O^{7}$ (YMC) [22].

DISTRIBUTION. Circum-Holarctic boreo-nemoral range [Mikhailov, 1997; Helsdingen, 2010; Platnick, 2010].

Araneus quadratus Clerck, 1757

MATERIAL. 5 ㅇ (TNU-2641/3) [41].

DISTRIBUTION. Trans-Palaearctic boreo-nemoral range [Mikhailov, 1997; Helsdingen, 2010; Platnick, 2010].

Araniella cucurbitina (Clerck, 1757)

MATERIAL. $2 O^{7} O^{\top}$ (TNU-2652/20) [54].

DISTRIBUTION. West-Central Palaearctic nemoral range (from Europe to Central Asia) [Mikhailov, 1997; Helsdingen, 2010; Platnick, 2010].

Argiope bruennichi (Scopoli, 1772)

RECORDS. Akhali Atoni, Sukhum [Spassky, 1937; Mcheidze, 1964, 1997; Otto \& Dietzold, 2006].

MATERIAL. 2 우 (TNU-2651/51) [55].

DISTRIBUTION. Trans-Palaearctic nemoral range [Mikhailov, 1997; Helsdingen, 2010; Platnick, 2010].

Atea sturmi (Hahn, 1831)

MATERIAL. 3 ㅇ (TNU-2652/21), 1 (YMC) [54].

DISTRIBUTION. West-Central Palaearctic nemoral range, from Europe to Yenisei [Mikhailov, 1997; Helsdingen, 2010; Platnick, 2010]. 
Cercidia prominens (Westring, 1851)

MATERIAL. 1 ( (YMC) [27]; 2 ㅇ (TNU-2653/4) [56].

DISTRIBUTION. Circum-Holarctic boreo-nemoral range [Mikhailov, 1997; Helsdingen, 2010; Platnick, 2010].

Cyclosa oculata (Walckenaer, 1802)

RECORDS. Sukhum [Spassky, 1937; Mcheidze, 1997; Otto \& Dietzold, 2006].

DISTRIBUTION. West-Palaearctic nemoral range [Mikhailov, 1997; Helsdingen, 2010; Platnick, 2010].

Cyrtarachne ixoides (Simon, 1870)

RECORDS. Gagra, Sukhum [Spassky, 1937 - sub $C$. ixodoides; Mcheidze, 1964, 1997; Otto \& Dietzold, 2006].

MATERIAL. 1 (TNU-2636/5) [36]; 1 (TNU-2651/48) [55].

DISTRIBUTION. Mediterranean to Georgia, Madagascar [Mikhailov, 1997; Helsdingen, 2010; Platnick, 2010].

Hypsosinga albovittata (Westring, 1851)

RECORDS. Gagra [Mcheidze, 1997; Otto \& Dietzold, 2006].

DISTRIBUTION. Trans-Palaearctic polyzonal range [Mikhailov, 1997; Helsdingen, 2010; Platnick, 2010].

Hypsosinga pygmaea (Sundevall, 1831)

RECORDS. Sukhum [Mcheidze, 1997; Otto \& Dietzold, 2006].

DISTRIBUTION. Circum-Holarctic boreo-nemoral range [Mikhailov, 1997; Helsdingen, 2010; Platnick, 2010].

\section{Hypsosinga sanguinea (C.L. Koch, 1844)}

RECORDS. Sukhum [Spassky, 1937; Mcheidze, 1997; Otto \& Dietzold, 2006].

MATERIAL. 1 (TNU-2636/4) [36].

DISTRIBUTION. Trans-Palaearctic boreo-nemoral range [Mikhailov, 1997; Helsdingen, 2010; Platnick, 2010].

\section{Larinioides ixobolus (Thorell, 1873)}

MATERIAL. 2 오 (TNU-2636/9) [36].

DISTRIBUTION. West and Central Palaearctic nemoral range: known from Germany east to South Siberia, and from Finland south to Bulgaria and Middle Asian Mts. [Mikhailov, 1997; Helsdingen, 2010; Platnick, 2010].

Mangora acalypha (Walckenaer, 1802)

RECORDS. Kelasur, Sukhum [Spassky, 1937; Mcheidze, 1964, 1997; Otto \& Dietzold, 2006].
MATERIAL. 2 OO (CP-27.16.1/11) [02]; $1 \sigma^{\top}, 11$ o (TNU2636/3) [36]; 2 우 (TNU-2638/1) [38]; 6 우 (TNU-2639/4) [39]; 20 우 (TNU-2651/49) [55]; 18 우 (TNU-2653/2) [56]; 1 (TNU2655/2) [58].

DISTRIBUTION. West and Central Palaearctic nemoral range: from Portugal east to Yenisei River [Mikhailov, 1997; Helsdingen, 2010; Platnick, 2010].

Neoscona adianta (Walckenaer, 1802)

RECORDS. Sukhum [Spassky, 1937 and Mcheidze, 1997 — sub Araneus; Otto \& Dietzold, 2006].

DISTRIBUTION. Trans-Palaearctic nemoral range [Mikhailov, 1997; Helsdingen, 2010; Platnick, 2010].

Neoscona subfusca (C.L. Koch, 1873)

RECORDS. Sukhum [Spassky, 1937 and Mcheidze, 1997 - sub Araneus dalmaticus Cl.; Otto \& Dietzold, 2006]. [38].

MATERIAL. 1 (TNU-2636/7) [36]; 2 우 (TNU-2638/2)

DISTRIBUTION. Ancient Mediterranean range: from Iberian Peninsula to western Turkmenistan [Mikhailov, 1997; Helsdingen, 2010; Platnick, 2010].

Nuctenea umbratica (Clerck, 1757)

MATERIAL. $4 \bigcirc^{7} \sigma^{7}, 26$ + 9 (TNU-2636/8) [36]; 19 (CP) [52];

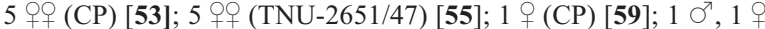
(CP) [62]; 1 O (CP) [63]

DISTRIBUTION. West-Palaearctic nemoral range [Mikhailov, 1997; Helsdingen, 2010; Platnick, 2010].

Singa nitidula (C.L. Koch, 1844)

RECORDS. Gagra; Gudauta; Sukhum [Spassky, 1937; Mcheidze, 1997; Otto \& Dietzold, 2006].

DISTRIBUTION. Trans-Palaearctic nemoral range [Mikhailov, 1997; Helsdingen, 2010; Platnick, 2010].

Zilla diodia (Walckenaer, 1802)

RECORDS. Akhali Atoni, Gudauta, Sukhum [Mcheidze, 1964, 1997; Otto \& Dietzold, 2006]. [56].

MATERIAL. 2 우 (TNU-2651/50) [55]; 2 우 (TNU-2653/3)

DISTRIBUTION. West-Palaearctic nemoral range, from Europe to East Kazakhstan [Mikhailov, 1997; Helsdingen, 2010; Platnick, 2010].

ATYPIDAE (1 genus and 1 species)

Atypus muralis Bertkau, 1890

MATERIAL. $1 O^{7}(\mathrm{CP})$ [59].

DISTRIBUTION. Central Europe to Turkmenistan nemoral range: from Germany and Italy to Urals and Kopetdagh Mts. [Mikhailov, 1997; Helsdingen, 2010; Platnick, 2010]. 
CHEIRACANTHIIDAE (1 genus and 2 species)

Cheiracanthium erraticum (Walckenaer, 1802)

RECORDS. Sukhum [Spasski, 1937; Mcheidze, 1997; Otto \& Dietzold, 2006].

MATERIAL. $40^{7} \sigma^{7}, 4$ 우 (TNU-2652/28) [54].

DISTRIBUTION. Trans-Palaearctic boreo-nemoral range [Mikhailov, 1997; Helsdingen, 2010; Platnick, 2010].

\section{Cheiracanthium mildei L. Koch, 1864}

RECORDS. Ritsa Lake [Mcheidze, 1997; Otto \& Dietzold, 2006].

MATERIAL. 1 (YMC) [08]; $1 \bigcirc^{7}$ (TNU-2636/49) [36]; 19 (CP) [59].

DISTRIBUTION. Nearctic - West-Central Palaearctic nemoral range and Argentina [Mikhailov, 1997; Helsdingen, 2010; Platnick, 2010].

CLUBIONIDAE (1 genus and 6 species)

Clubiona brevipes Blackwall, 1841

MATERIAL. 1 (TNU-2654/2) [57].

DISTRIBUTION. Plaearctic disjunctive nemoral range, it is absent between Caucasus and Japan [Mikhailov, 1997; Helsdingen, 2010; Platnick, 2010].

Clubiona caerulescens L.Koch, 1867

MATERIAL. 1 (TNU-2639/20) [39].

DISTRIBUTION. Trans-Palaearctic boreo-nemoral range [Mikhailov, 1997; Helsdingen, 2010; Platnick, 2010].

Clubiona germanica Thorell, 1871

RECORDS. Gagra [Mcheidze, 1997; Otto \& Dietzold, 2006].

DISTRIBUTION. Trans-Palaearctic boreo-nemoral range [Mikhailov, 1997; Helsdingen, 2010; Platnick, 2010].

\section{Clubiona lutescens Westring, 1851}

RECORDS. Myussera State Reserve [Mikhailov, 1990; Otto \& Dietzold, 2006].

MATERIAL. 1 + (TNU-2636/51) [36]; $3 \sigma^{7} \sigma^{7}, 3$ 우 (TNU2639/21) [39]; $1 \mathrm{O}^{\top}, 4$ 우 (TNU-2640/2) [40]; $3 \mathrm{O}^{\top} \mathrm{O}^{\lambda}, 4$ 우우 (TNU2651/35) [55]; 2 ᄋ ○ $^{7}, 4$ 우 (TNU-2653/9) [56].

DISTRIBUTION. Holarctic nemoral range [Mikhailov, 1997; Helsdingen, 2010; Platnick, 2010].

Clubiona neglecta O. Pickard-Cambridge, 1862

MATERIAL. $3 \bigcirc^{7} \sigma^{7}, 6$ 우 (TNU-2652/29) [54].

DISTRIBUTION. Trans-Palaearctic nemoral range [Mikhailov, 1997; Helsdingen, 2010; Platnick, 2010].
Clubiona pseudosimilis Mikhailov, 1990

MATERIAL. $1 \bigcirc^{\top}, 4$ 우 (CP-20.10.16/1) [01]; $4 \bigcirc^{7} \sigma^{7}, 16$ 우 (TNU-2641/20) [41].

DISTRIBUTION. Caucasus [Mikhailov, 1997].

COELOTIDAE (1 genus and 1 species)

Pireneitega (sub Paracoelotes) sp.

MATERIAL. 6 O $^{7} O^{\top}, 1$ ㅇ (CP-22.12.5/2) [33]; 11 우 (TNU2640/12) [40]; $2 O^{7} O^{7}, 78$ 우 (TNU-2641/1) [41]; 3 우 (TNU2642/1) [43]; 1 क (TNU-2644/16) [45]; 64 ㅇ (TNU-2652/2) [54].

NOTE. This is a new species closely related to Pireneitega segestriformis (Dufour, 1820). Its description will be published in a separate paper.

Earlier this genus was placed in Amaurobiidae [see Platnick, 2010]. But recently Y. Nishikawa [2009] established the separate family Coelotidae (new status for the subfamily). We agree with Y. Nishikawa's opinion.

DISTRIBUTION. West Caucasus [personal data].

CORINNIDAE (2 genera and 2 species)

Phrurolithus festivus (C.L. Koch, 1835)

MATERIAL. 1 ( $(\mathrm{CP})[\mathbf{6 2}]$.

DISTRIBUTION. Trans-Palaearctic nemoral range [Mikhailov, 1997; Helsdingen, 2010; Platnick, 2010].

Trachelas minor O. Pickard-Cambridge, 1872

RECORDS. Gudauta and Sukhum Distr. [Kovblyuk \& Nadolny, 2009; Marusik \& Kovblyuk, 2010].

MATERIAL. $1 \sigma^{7}$ (YMC) [10]; $15 \sigma^{7}+$ (YMC) [11]; 1 ㅇ (TNU-2636/1) [36]; 1 Oे $^{\text {T }}$ TNU-2653/68) [56].

DISTRIBUTION. West Palaearctic nemoral range [Mikhailov, 1997; Helsdingen, 2010; Platnick, 2010].

CYBAEIDAE (1 genus and 1 species)

Cybaeus abchasicus Charitonov, 1947*

RECORDS. Gudauta Distr., Tarkikadze Cave [Charitonov, 1947; Dzhanashvilli, 1965; Mcheidze, 1997; Otto \& Dietzold, 2006; Kovblyuk et al., 2010].

MATERIAL. $1 O^{\top}$ (ZMMU) [09].

DISTRIBUTION. West Caucasus [Kovblyuk et al., 2010].

DICTYNIDAE (2 genera and 2 species)

Dictyna uncinata Thorell, 1856

MATERIAL. $1 O^{7}$ (CP-34.14.4/34) [34]; $1 O^{7}$ (CP) [52].

DISTRIBUTION. Trans-Palaearctic nemoral range [Mikhailov, 1997; Helsdingen, 2010; Platnick, 2010].

Mastigusa macrophthalma (Kulczyński, 1897)

MATERIAL. 1 (YMC) [08]. 
DISTRIBUTION. West and Central Palaearctic nemoral range: from France east to Yenisei River, and from Britain south to Italy and Bulgaria [Helsdingen, 2010; Platnick, 2010].

DYSDERIDAE (3 genera and 11 species)

?? Dysdera cribrata Simon 1882

RECORDS. Akhali Atoni, Sukhum [Mcheidze, 1964, 1997; Otto \& Dietzold, 2006].

NOTE. Probably the record from Caucasus is based on misidentification.

DISTRIBUTION. France [Helsdingen, 2010; Platnick, 2010].

Dysdera crocata C.L. Koch, 1838

RECORDS. Sukhum Distr.: Nizhnyaya Yashtukha Vil.; Kelasur; Ochamchyra; Kodor [Dunin, 1992b; Mcheidze, 1997; Otto \& Dietzold, 2006].

DISTRIBUTION. Cosmopolitan, but absent in Asia [Mikhailov, 1997; Helsdingen, 2010; Platnick, 2010].

Dysdera dunini Deeleman-Reinhold, 1988

RECORDS. Gudauta Distr.; Bzyb Range; Sukhum [Dunin, 1992b; Mcheidze, 1997; Otto \& Dietzold, 2006]. Sub Dysdera punctata C.L. Koch, 1838 - misidentification [see Dunin, 1992b]: Gagra; Kodor [Mcheidze, 1997; Otto \& Dietzold, 2006].

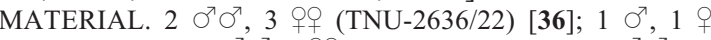
(TNU-2652/15) [54]; 8 ○ $^{7}, 4$ 우 (TNU-2651/30) [55]; $5 \sigma^{7} \sigma^{7}, 11$ 우 (TNU-2653/23) [56]; $1 \mathrm{O}^{7}$ (TNU-2654/1) [57]; 1 ㅇ (CP) [62].

DISTRIBUTION. Crimea-Caucasus and probably Middle Asian Mts. [Mikhailov, 1997; Kovblyuk et al., 2008; Helsdingen, 2010; Platnick, 2010].

?? Dysdera erythrina (Walckenaer, 1802)

RECORDS. Akhali Atoni, Gagra, Sukhum [Mcheidze, 1964, 1997; Otto \& Dietzold, 2006].

NOTE. Probably this record is based on misidentification [see Kovblyuk et al., 2009].

DISTRIBUTION. Europe west from Carpathians [Kovblyuk et al., 2008].

Dysdera hungarica Kulczyński in Chyzer et Kulczyński, 1897

MATERIAL. $1 \sigma^{\top}, 5$ 우 (TNU-2639/16) [39]; $1 \sigma^{\top}, 2$ 우 (TNU-2644/28) [45].

DISTRIBUTION. East-European-Balkans-CrimeaCaucasian mountain ranges [Mikhailov, 1997; Kovblyuk et al., 2008; Helsdingen, 2010; Platnick, 2010].

Dysdera lata Wider in Reuss, 1834

MATERIAL. $1 \bigcirc^{7}, 3$ ㅇ (TNU-2651/29) [55].
DISTRIBUTION. Mediterranean-Caucasian range: Corsica, Greece, Cyprus, Moldova, Ukraine (Odessa, Mykolaiv and Kherson Area, Crimea), Turkey, West Caucasus [Mikhailov, 1997; Kovblyuk et al., 2008; Helsdingen, 2010; Platnick, 2010].

Dysdera martensi Dunin, 1991

RECORDS. Gudauta; Bzyb Mt. Range [Dunin, 1992b; Otto \& Dietzold, 2006].

DISTRIBUTION. Caucasus [Mikhailov, 1997; Platnick, 2010].

Harpactea caucasia (Kulczyński, 1895)

RECORDS. Gudauta and Sukhum Distr. [Dunin, 1992b; Otto \& Dietzold, 2006].

MATERIAL. $10^{7}$ (TNU-2515/2) [31]; 4 우우 (TNU-2636/24) [36]; $4 \bigcirc^{\top} \bigcirc^{\top}, 11$ 우 (TNU-2639/15) [39]; 2 우 (TNU-2644/27) [45]; $1 \bigcirc^{\top}, 36$ 우 (TNU-2651/28) [55]; 1 ○ $^{7}, 20$ 우 (TNU-2653/22) [56].

DISTRIBUTION. Caucasus [Mikhailov, 1997; Helsdingen, 2010; Platnick, 2010].

Harpactea logunovi Dunin, 1992

RECORDS. Bzyb Mt. Range, Pitsunda, Myusser Reserve, Sukhum [Dunin, 1992a,b; Otto \& Dietzold, 2006].

MATERIAL. $1 O^{7}$ (CP-43.11.2/1) [05]; $1 O^{7}, 2$ 오 (TNU-2644/ 26) $[45] ; 1$ ㅇ (CP-43.11.2/6) [49]; 3 O잉, 31 우 (TNU-2652/17) [54]; 1 O, 9 우 (TNU-2653/21) [56]; 1 \& (CP) [62].

DISTRIBUTION. Caucasus [Mikhailov, 1997; Helsdingen, 2010; Platnick, 2010].

Harpactea rubicunda (C.L. Koch, 1838)

RECORDS. Bzyb Mt. Range, Khuach Vil. [Dunin, 1992b].

MATERIAL. 1 ( $(\mathrm{CP}-43.11 .3 / 1)$ [09].

DISTRIBUTION. West Palaearctic nemoral range: from Spain east to Caucasus, and from Britain south to Greece [Mikhailov, 1997; Kovblyuk et al., 2008; Helsdingen, 2010; Platnick, 2010].

Hygrocrates caucasicus Dunin, 1992*

RECORDS. Gudauta Distr.: Achandara Vil., Khuach Vil. [Dunin, 1992b; Otto \& Dietzold, 2006].

DISTRIBUTION. West Caucasus [Mikhailov, 1997; Helsdingen, 2010; Platnick, 2010].

FILISTATIDAE (1 genus and 1 species)

Pritha sp.

MATERIAL. 5 우 (YMC) [06]; $1 \bigcirc^{7}$ (TNU-2636/2) [36]; 1 ㅇ (TNU-2651/7) [55].

NOTE. Probably undescribed species. Its description will be published in a separate paper.

DISTRIBUTION. Abkhazia [personal data]. 
GNAPHOSIDAE ( 8 genera and 13 species)

Drassodes lapidosus (Walckenaer, 1802)

RECORDS. All sea shore [Wagner, 1896 — sub Drassus lapidicola Walck.]; Kelasur, Sukhum [Mcheidze, 1964]

MATERIAL. 2 우 (TNU-2641/19) [41]; 77 우 (TNU-2652/ 30) $[\mathbf{5 4}]$.

DISTRIBUTION. West-Palaearctic nemoral range [Mikhailov, 1997; Helsdingen, 2010; Platnick, 2010].

Drassodes pubescens (Thorell, 1856)

MATERIAL. 1 (TNU-2652/31) [54].

DISTRIBUTION. Trans-Palaearctic nemoral range [Mikhailov, 1997; Helsdingen, 2010; Platnick, 2010].

Drassyllus pusillus (C.L. Koch, 1833)

MATERIAL. 1 (TNU-2636/31) [36]; $1 O^{7}, 1$ (TNU-2640/

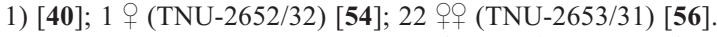

DISTRIBUTION. Trans-Palaearctic nemoral range [Mikhailov, 1997; Helsdingen, 2010; Platnick, 2010].

Gnaphosa dolosa Herman, 1879

RECORDS. Gentsvishi, Sukhum [Ovtsharenko et al, 1992; Esyunin \& Efimik, 1997; Kovblyuk, 2005].

DISTRIBUTION. West and Central Palaearctic nemoral range [Mikhailov, 1997; Helsdingen, 2010; Platnick, 2010].

Gnaphosa lugubris (C.L. Koch, 1839)

RECORDS. Gagra [Simon, 1899; Verzhbitsky, 1902; Charitonov, 1932; Ovtsharenko, 1982; Mcheidze, 1997; Otto \& Dietzold, 2006].

DISTRIBUTION. West and Central Palaearctic nemoral range [Mikhailov, 1997; Helsdingen, 2010; Platnick, 2010].

Haplodrassus signifer (C.L. Koch, 1839)

MATERIAL. 3 우 (TNU-2652/35) [54].

DISTRIBUTION. Circum-Holarctic boreo-nemoral range [Mikhailov, 1997; Helsdingen, 2010; Platnick, 2010].

Kishidaia conspicua (L. Koch, 1866)

MATERIAL. $10^{7}, 1$ ( 1 (TNU-2653/30) [56].

DISTRIBUTION. West and Central Palaearctic nemoral range [Mikhailov, 1997; Helsdingen, 2010; Platnick, 2010].

Scotophaeus blackwalli (Thorell, 1871)

MATERIAL. $10 \odot^{7} \odot^{7}, 16$ ㅇ (YMC) [08].

DISTRIBUTION. Cosmopolitan, but in the Palaearctic it is known in Europe only [Mikhailov, 1997; Helsdingen, 2010; Platnick, 2010].
Scotophaeus scutulatus (L. Koch, 1866)

RECORDS. Sukhum [Mcheidze, 1997 - sub $S$. acutularis; Otto \& Dietzold, 2006].

DISTRIBUTION. West and Central Palaearctic nemoral range [Mikhailov, 1997; Helsdingen, 2010; Platnick, 2010].

Trachyzelotes pedestris (C.L. Koch, 1837)

MATERIAL. $1 O^{\top}$ (TNU-2653/32) [56].

DISTRIBUTION. West-Palaearctic nemoral range [Mikhailov, 1997; Helsdingen, 2010; Platnick, 2010].

Zelotes caucasius (L. Koch, 1866)

RECORDS. Gagra [Simon, 1899 - sub Melanophora; Verzhbitsky, 1902 - sub Prosthesima; Ovtsharenko, 1982; Otto \& Dietzold, 2006].

DISTRIBUTION. West and Central Palaearctic nemoral range [Mikhailov, 1997; Helsdingen, 2010; Platnick, 2010].

Zelotes khostensis Kovblyuk et Ponomarev, 2008

RECORDS. Sukhum [sub Zelotes erebeus (Thorell, 1871) - misidentification [see Kovblyuk \& Ponomarev, 2008] — Mcheidze, 1997; Otto \& Dietzold, 2006].

MATERIAL. $1 O^{7}$ (TNU-2639/11) [39]; $10^{7}$ (TNU-2644/4) [45]; $2 \bigcirc^{7} \bigcirc^{7}, 2$ 우 (TNU-2651/12) [55]; $2 \bigcirc^{7} \bigcirc^{7}, 2$ 우 (TNU-2653/29) [56].

DISTRIBUTION. Caucasus [personal data].

Zelotes subterraneus (C.L. Koch, 1833)

MATERIAL. $1 \sigma^{7}, 1$ ( 1 (CP-18.14.17/21) [09]; 1 (TNU2636/30) [36]; 1 ㅇ (TNU-2639/12) [39]; $2 \mathrm{O}^{7} \sigma^{7}, 12$ 우 (TNU2641/2) [41]; 1 O $^{\top}, 9$ 우 (TNU-2651/11) [55]; $3 \bigcirc^{7} \bigcirc^{7}, 8$ 우 (TNU2653/28) [56]; 1 ㅇ (CP) [62].

DISTRIBUTION. West and Central Palaearctic nemoral range [Mikhailov, 1997; Helsdingen, 2010; Platnick, 2010; personal data].

HAHNIIDAE ( 1 genus and 1 species)

Hahnia nava (Blackwall, 1841)

MATERIAL. $34 \circ^{7}+$ (YMC) [28]; 1 ( (TNU-2653/67) [56].

DISTRIBUTION. Trans-Palaearctic nemoral range [Mikhailov, 1997; Helsdingen, 2010; Platnick, 2010].

Hahnia sp. cf. montana (Blackwall, 1841)

MATERIAL. $5 \bigcirc^{7} \odot^{7}, 4$ 4 (YMC) [24]; 9 $९$ (TNU-2636/46) [36]; 4 우 (TNU-2639/3) [39]; 3 우 (TNU-2651/25) [55]; 1 우 (TNU-2653/66) [56].

NOTE. Probably it is an undescribed species.

DISTRIBUTION. West Caucasus [personal data].

LEPTONETIDAE (1 genus and 1 species)

Leptonetela caucasica Dunin, 1990

MATERIAL. $1 \sigma^{r}, 1$ (YMC) [24]. 
DISTRIBUTION. West Caucasus [Mikhailov, 1997; Marusik \& Guseinov, 2003; Ponomarev \& Chumachenko, 2007; Helsdingen, 2010; Platnick, 2010].

NOTE. Probably, record of this species from Azerbaijan is doubtful [personal data].

LINYPHIIDAE (43 genera and 64 species)

Agyneta conigera (O. Pickard-Cambridge, 1863)

MATERIAL. 3 + $($ (VAG) [41]; 1 (VAG) [54].

DISTRIBUTION. Trans-Palaearctic boreo-nemoral range [Mikhailov, 1997; Helsdingen, 2010; Platnick, 2010].

Agyneta ramosa Jackson, 1912

MATERIAL. $10^{7}, 4$ ㅇ (VAG) [54].

DISTRIBUTION. West and Central Palaearctic boreo-nemoral range [Mikhailov, 1997; Helsdingen, 2010; Platnick, 2010].

Allotiso lancearius (Tanasevitch, 1987)

RECORDS. Tkuarchal Distr., Khodzhal [Tanasevitch, 1990].

MATERIAL. 1 ( $(\mathrm{VAG})[41]$.

DISTRIBUTION. West Caucasus [Mikhailov, 1997; Platnick, 2010].

Araeoncus caucasicus Tanasevitch, 1987

MATERIAL. $1 \sigma^{7}$ (YMC) [20]; $1 \sigma^{7}, 1$ (VAG) [36]; $1 \sigma^{7} \sigma^{7}, 1$ ๆ (VAG) [39]; 1 \% (VAG) [55].

DISTRIBUTION. Crimea, Rostov Area of Russia, Caucasus, Iran, West Kazakhstan and Middle Asian Mts. [Mikhailov, 1997; Helsdingen, 2010; Platnick, 2010; Ponomarev, personal data].

Bathyphantes approximatus (O. Pickard-Cambridge, 1871)

MATERIAL. 1 (YMC) [06].

DISTRIBUTION. West and Central Palaearctic boreo-nemoral range [Mikhailov, 1997; Helsdingen, 2010; Platnick, 2010].

Bathyphantes gracilis (Blackwall, 1841)

MATERIAL. $1 \bigcirc^{\top}, 1+$ (YMC) [06]; $1 \overbrace{}^{\top}, 1+$ (VAG) [55]; 3 $\bigcirc^{7} O^{7}, 5$ 우 (VAG) [56].

DISTRIBUTION. Circum-Holarctic boreo-nemoral range [Mikhailov, 1997; Helsdingen, 2010; Platnick, 2010].

Caucasopisthes procurvus (Tanasevitch, 1987)

MATERIAL. $10^{7}$ (VAG) [41].

DISTRIBUTION. Caucasus [Mikhailov, 1997; Helsdingen, 2010; Platnick, 2010].
Centromerus minor Tanasevitch, 1990

RECORDS. Gagra Distr.: Pitsunda, Bzyb River Valley [Tanasevitch, 1990; Otto \& Dietzold, 2006]. [54].

MATERIAL. 1 \% (VAG) [36]; 2 우 (VAG) [39]; 1 (VAG)

DISTRIBUTION. Caucasus, Turkey, Central Asian Mts [Mikhailov, 1997; Platnick, 2010].

Ceratinella brevis (Wider, 1834)

RECORDS. Gudauta Distr.: Myussera Reserve [Tanasevitch, 1987; Otto \& Dietzold, 2006].

MATERIAL. $7 \sigma^{7} \sigma^{7}, 5$ OP (YMC) [07]; $1 \sigma^{7}$ (YMC) [10]; 9

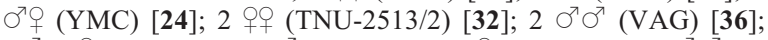
$10^{7}, 1$ ( (VAG) [41]; $1 \mathrm{O}^{7}$ (VAG) [43]; 1 (VAG) [44]; 5 O $^{7} \mathrm{O}^{7}, 13$ 우 (VAG) [54]; $1 \sigma^{\prime}, 1$ ( $(\mathrm{VAG})[\mathbf{5 5}] ; 1$ ( $(\mathrm{CP})[\mathbf{6 1}]$.

DISTRIBUTION. Trans-Palaearctic boreo-nemoral range [Mikhailov, 1997; Helsdingen, 2010; Platnick, 2010].

Cresmatoneta mutinensis (Canestrini, 1868)

RECORDS. Sukhum [Spassky, 1937 - sub Formicina; Otto \& Dietzold, 2006].

MATERIAL. 1 (YMC) [12].

DISTRIBUTION. Mediterranean-Crimea-Caucasian range [Mikhailov, 1997; Helsdingen, 2010; Platnick, 2010].

Diplocephalus caucasicus Tanasevitch, 1987*

RECORDS. Gudauta Distr.: Myussera Reserve; Sukhum, Kelasur Cave [Tanasevitch, 1987, 1990; Otto \& Dietzold, 2006]. [55].

MATERIAL. 2 우 (VAG) [37]; 1 (VAG) [43]; 1 ㅇ (VAG)

DISTRIBUTION. Caucasus [Mikhailov, 1997; Helsdingen, 2010; Platnick, 2010]. 1863)

Diplocephalus latifrons (O. Pickard-Cambridge,

RECORDS. Gagra Distr.: Pitsunda, Bzyb River Valley; Gudauta Distr.: Ritsa Lake [Tanasevitch, 1987; Tanasevitch, 1990; Otto \& Dietzold, 2006].

DISTRIBUTION. West Palaearctic nemoral range [Mikhailov, 1997; Helsdingen, 2010; Platnick, 2010].

Diplocephalus picinus (Blackwall, 1841)

MATERIAL. 2 ㅇ (VAG) [54].

DISTRIBUTION. West and Central Palaearctic nemoral range [Mikhailov, 1997; Helsdingen, 2010; Platnick, 2010].

Diplocephalus transcaucasicus Tanasevitch, 1990

MATERIAL. 4 ㅇ (VAG) [54].

DISTRIBUTION. Caucasus [Mikhailov, 1997; Helsdingen, 2010; Platnick, 2010]. 
Diplostyla concolor (Wider, 1834)

RECORDS. Gagra Distr.: Pitsunda, Bzyb River Valley; Gudauta Distr.: Myussera Reserve [Tanasevitch, 1987; Otto \& Dietzold, 2006].

MATERIAL. 2 O $^{7} \sigma^{7}, 3$ ㅇ (VAG) [36]; $10^{7}$ (VAG) [39]; 1 ㅇ (CP-39.14.3/22) [49]; $4 \mathrm{O}^{7} \sigma^{7}, 2$ 우 (VAG) [55].

DISTRIBUTION. Circum-Holarctic nemoral range (in Palaearctic east to Yenisei River) [Mikhailov, 1997; Helsdingen, 2010; Platnick, 2010].

Erigone dentipalpis (Wider, 1834)

RECORDS. Sukhum [Spassky, 1937; Mcheidze, 1997; Otto \& Dietzold, 2006].

MATERIAL. 2 ㅇ (VAG) [36]; 1 ( $(\mathrm{VAG})[\mathbf{4 1}] ; 1 \mathrm{O}^{\top}, 1$ ㅇ (VAG) $[\mathbf{4 5}] ; 7 \circlearrowleft^{7} \sigma^{7}, 1+$ (VAG) [55]; $14 \bigcirc^{7} \sigma^{7}, 3$ + + (VAG) [56]; 1 $\mathrm{O}^{7}(\mathrm{CP})[\mathbf{5 9}]$.

DISTRIBUTION. Trans-Palaearctic polyzonal range [Mikhailov, 1997; Marusik et al., 2000; Helsdingen, 2010; Platnick, 2010].

Erigone longipalpis (Sundevall, 1830)

MATERIAL. 2 ㅇ (VAG) [41]; $30^{7} \sigma^{7}$ (VAG) [42].

DISTRIBUTION. Trans-Palaearctic boreo-nemoral range [Mikhailov, 1997; Helsdingen, 2010; Platnick, 2010].

\section{Floronia bucculenta (Clerck, 1757)}

RECORDS. Sukhum [Tanasevitch, 1990, referring to S.A. Spassky [1937 — sub frenata (Wider, 1834)]; however, Spassky did not indicate this species for Abkhazia; Otto \& Dietzold, 2006].

DISTRIBUTION. Palaearctic nemoral range [Mikhailov, 1997; Helsdingen, 2010; Platnick, 2010].

\section{Frontinellina frutetorum (C.L. Koch, 1834)}

RECORDS. Sukhum [Spassky, 1937 - sub Linyphia; Mcheidze, 1964, 1997; Otto \& Dietzold, 2006].

MATERIAL. 1 ( $(\mathrm{VAG})$ [47].

DISTRIBUTION. West and Central Palaearctic nemoral range [Mikhailov, 1997; Helsdingen, 2010; Platnick, 2010].

Gnathonarium dentatum (Wider, 1834)

RECORDS. Sukhum [Spassky, 1937; Tanasevitch, 1987; Mcheidze, 1997; Otto \& Dietzold, 2006].

MATERIAL. $1 \mathrm{O}^{7}$ (VAG) [39]; $4 \mathrm{O}^{7} \sigma^{7}, 4$ 우 (VAG) [45]; 2 우우 (VAG) $[55] ; 3 \bigcirc^{7} \bigcirc^{7}, 5$ ㅇ (VAG) [56].

DISTRIBUTION. Trans-Palaearctic nemoral range [Mikhailov, 1997; Helsdingen, 2010; Platnick, 2010].

Gonatium rubens (Blackwall, 1833)

RECORDS. Gudauta Distr.: Ritsa Lake [Tanasevitch, 1990].
DISTRIBUTION. Trans-Palaearctic boreo-nemoral range [Mikhailov, 1997; Helsdingen, 2010; Platnick, 2010].

\section{Gongylidiellum murcidum Simon, 1884}

RECORDS. Gudauta Distr.: Myussera Reserve and Ritsa Lake; Sukhum Distr.: Kelasur [Tanasevitch, 1987; Tanasevitch, 1990; Otto \& Dietzold, 2006].

DISTRIBUTION. Palaearctic disjunctive nemoral range, it is unknown between Caucasus and Japan [Mikhailov, 1997; Helsdingen, 2010; Platnick, 2010]. 1875)

Gongylidiellum vivum (O. Pickard-Cambridge,

MATERIAL. 1 (VAG) [56].

DISTRIBUTION. Palaearctic disjunctive nemoral range, it is unknown between Caucasus and Far East (Sakhalin) [Mikhailov, 1997; Helsdingen, 2010; Platnick, 2010].

Hylyphantes nigritus (Simon, 1881)

MATERIAL. 7 우 (VAG) [39]; 11 우 (VAG) [45]; $10^{7}, 6$ 우 (VAG) [56].

DISTRIBUTION. Trans-Palaearctic nemoral range [Mikhailov, 1997; Marusik et al., 2000; Helsdingen, 2010; Platnick, 2010].

Ipa keyserlingi (Ausserer, 1867)

MATERIAL. $10^{7}$ (YMC) [28].

DISTRIBUTION. West Palaearctic nemoral range [Mikhailov, 1997; Helsdingen, 2010; Platnick, 2010].

Lepthyphantes cruentatus Tanasevitch, 1987

MATERIAL. $1+$ (VAG) [55].

DISTRIBUTION. Caucasus [Mikhailov, 1997; Helsdingen, 2010; Platnick, 2010].

Linyphia hortensis Sundevall, 1830 [54].

MATERIAL. 3 † (VAG) [40]; 1 (VAG) [44]; 4 + (VAG)

DISTRIBUTION. Trans-Palaearctic nemoral range [Mikhailov, 1997; Helsdingen, 2010; Platnick, 2010].

Linyphia triangularis (Clerck, 1757)

MATERIAL. $3 O^{7} O^{7}$ (VAG) [47].

DISTRIBUTION. Palaearctic nemoral range, introduced in USA [Mikhailov, 1997; Helsdingen, 2010; Platnick, 2010].

Maso sundevalli (Westring, 1851)

RECORDS. Gudauta Distr.: Ritsa Lake [Tanasevitch, 1990].

MATERIAL. $20^{7} \sigma^{7}, 6$ + (VAG) [41]; 1 (VAG) [45]. 
DISTRIBUTION. Circum-Holarctic boreo-nemoral range [Mikhailov, 1997; Marusik et al., 2000; Helsdingen, 2010; Platnick, 2010].

Meioneta mollis (O. Pickard-Cambridge, 1871)

RECORDS. Abkhazia, unspecified [Tanasevitch, 1990 - sub Agyneta; Otto \& Dietzold, 2006].

MATERIAL. 1 \% (YMC) [10]; 1 + (VAG) [39]; $4 O^{7} \sigma^{7}, 5$ 우 (VAG) [56].

DISTRIBUTION. Trans-Palaearctic nemoral range [Mikhailov, 1997; Helsdingen, 2010; Platnick, 2010].

\section{Meioneta rurestris (C.L. Koch, 1836)}

RECORDS. Sukhum: Niszhnaya Yashtukha [Tanasevitch, 1990; Otto \& Dietzold, 2006].

MATERIAL. $20^{7} \sigma^{7}, 6$ ㅇ (VAG) [41]; 2 +क (VAG) [54].

DISTRIBUTION. West-Palaearctic nemoral range [Mikhailov, 1997; Helsdingen, 2010; Platnick, 2010]. 1872)

Metopobactrus prominulus (O. Pickard-Cambridge,

MATERIAL. 3 우 (YMC) [12]; $1 \sigma^{7}, 4$ ㅇ (YMC) [27].

DISTRIBUTION. Circum-Holarctic boreo-nemoral range [Mikhailov, 1997; Marusik et al., 2000; Helsdingen, 2010; Platnick, 2010].

Micrargus herbigradus (Blackwall, 1854)

RECORDS. Gagra Distr.: Pitsunda, Bzyb River Valley [Tanasevitch, 1987; Otto \& Dietzold, 2006].

MATERIAL. 2 ㅇ (VAG) [39]; $10^{\text {T }}$ (VAG) [41].

DISTRIBUTION. Trans-Palaearctic boreo-nemoral range [Mikhailov, 1997; Helsdingen, 2010; Platnick, 2010].

Micrargus subaequalis (Westring, 1851)

MATERIAL. 2 + (YMC) [24]; 1 (VAG) [56].

DISTRIBUTION. Trans-Palaearctic nemoral range [Mikhailov, 1997; Helsdingen, 2010; Platnick, 2010].

Microneta viaria (Blackwall, 1841)

RECORDS. Gagra Distr.: Pitsunda, Bzyb River Valley and Ritsa Lake; Gudauta Distr.: Myussera Reserve [Tanasevitch, 1987; Tanasevitch, 1990; Otto \& Dietzold, 2006].

MATERIAL. $34 \sigma^{7}+$ (YMC) [07]; 2 + $(\mathrm{VAG})$ [32]; 1 (VAG) [36]; 20 오 (VAG) [39]; 5 오 (VAG) [41]; 2 오 (VAG) [45]; $1 \bigcirc^{7}, 8$ ㅇ (VAG) [54]; 4 우 (VAG) [55]; 2 우 (VAG) [56].

DISTRIBUTION. Circum-Holarctic boreo-nemoral range [Mikhailov, 1997; Helsdingen, 2010; Platnick, 2010].

Moebelia penicillata (Westring, 1851)

MATERIAL. 19 (YMC) [06]; 19 (YMC) [07]; $3 \sigma^{7} \sigma^{7}, 7$ 우 (YMC) [08].
DISTRIBUTION. West and Central Palaearctic nemoral range [Mikhailov, 1997; Helsdingen, 2010; Platnick, 2010].

Nematogmus sanguinolentus (Walckenaer, 1841)

MATERIAL. 1 (VAG) [39].

DISTRIBUTION. Trans-Palaearctic nemoral range [Mikhailov, 1997; Helsdingen, 2010; Platnick, 2010].

Neriene clathrata (Sundevall, 1830)

MATERIAL. $1 \uparrow(\mathrm{YMC})[\mathbf{0 6}] ; 1 \overbrace{}^{7}, 1 \%$ (YMC) [12]; 19 (YMC) [27].

DISTRIBUTION. Circum-Holarctic nemoral range [Mikhailov, 1997; Helsdingen, 2010; Platnick, 2010].

Neriene peltata (Wider, 1834)

MATERIAL. 1 (CP-39.10.7/14) [34]; $10^{7}, 6$ 우 (VAG) [39]; 1 ( $(\mathrm{VAG})[40] ; 2$ + (VAG) $[41] ; 1_{1} \bigcirc^{\prime}, 7$ 우 (VAG) [54].

DISTRIBUTION. Greenland and West Palearctic nemoral range [Mikhailov, 1997; Helsdingen, 2010; Platnick, 2010].

Neriene radiata (Walckenaer, 1841)

MATERIAL. 19 (CP-39.10.10/6) [02]; 2 + + (VAG) [36]; $2+9$ (VAG) [39].

DISTRIBUTION. Circum-Holarctic nemoral range [Mikhailov, 1997; Helsdingen, 2010; Platnick, 2010].

Obscuriphantes obscurus (Blackwall, 1841)

MATERIAL. $20^{7} \sigma^{7}, 1+$ (VAG) [41].

DISTRIBUTION. West and Central Palaearctic boreo-nemoral range [Mikhailov, 1997; Helsdingen, 2010; Platnick, 2010].

Oedothorax apicatus (Blackwall, 1850)

RECORDS. Niszhnaya Yashtukha [Tanasevitch, 1990; Otto \& Dietzold, 2006].

MATERIAL. $4 O^{7} \sigma^{7}, 1+$ (YMC) [11]; $1 \uparrow(\mathrm{VAG})[\mathbf{3 6}]$

DISTRIBUTION. Trans-Palaearctic nemoral range [Mikhailov, 1997; Helsdingen, 2010; Platnick, 2010].

Oedothorax meridionalis Tanasevitch, 1987*

RECORDS. Sukhum Distr.: Kelasur [Tanasevitch, 1987; Otto \& Dietzold, 2006].

MATERIAL. $1 \odot^{7}, 2$ + + (VAG) [36]; $1 \bigcirc^{7}, 2$ ㅇ (VAG) [39]; 1 \& (VAG) [45]; 1 + (VAG) [55].

DISTRIBUTION. Caucasus, Iran and Middle Asian Mts. [Mikhailov, 1997; Helsdingen, 2010; Platnick, 2010].

Parapelecopsis nemoralis (Blackwall, 1841)

MATERIAL. 1 (VAG) [55].

DISTRIBUTION. West Palaearctic nemoral range [Mikhailov, 1997; Helsdingen, 2010; Platnick, 2010]. 


\section{Plesiophantes sp.}

MATERIAL. 1 (VAG) [36]; $10^{7}, 3$ क + (VAG) [39]; $4 \odot^{7} \sigma^{7}, 6$ 우 (VAG) [41]; 5 OT $^{7}, 11$ 우 (VAG) [55].

NOTE. Undescribed species. Will be described by Gnelitsa in a separate paper.

DISTRIBUTION. West Caucasus.

\section{Porrhomma convexum (Westring, 1851)}

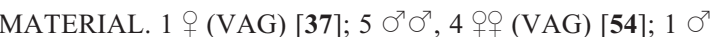
(VAG) [56].

DISTRIBUTION. West and Central Palaearctic boreo-nemoral range [Mikhailov, 1997; Helsdingen, 2010; Platnick, 2010].

\section{Porrhomma pygmaeum (Blackwall, 1834)}

RECORDS. Gudauta Distr.: Myussera Reserve [Tanasevitch, 1987; Otto \& Dietzold, 2006].

MATERIAL. $10^{7}$ (VAG) [56].

DISTRIBUTION. Trans-Palaearctic boreo-nemoral range [Mikhailov, 1997; Helsdingen, 2010; Platnick, 2010].

Prinerigone vagans (Audouin, 1826)

RECORDS. Sukhum Distr.: Nizhnyaya Yashtukha Vil. [Tanasevitch, 1990; Otto \& Dietzold, 2006]. [55].

MATERIAL. 1 (VAG) [36]; $10^{7}$ (VAG) [45]; 5 O $^{7} \mathrm{O}^{7}$ (VAG)

DISTRIBUTION. West and Central Palaearctic nemoral range [Mikhailov, 1997; Helsdingen, 2010; Platnick, 2010]. 2009*

Scutpelecopsis wunderlichi Marusik \& Gnelitsa,

RECORDS. Gagra Distr.: env. Pitsunda, Ldzaa Vil. [Marusik \& Gnelitsa, 2009].

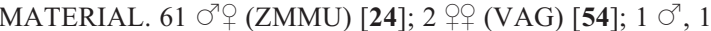
q (VAG) [55]; 1 \% (VAG) [56]; 1 ( (CP) [61].

NOTE. Records of Pelecopsis krausi Wunderlich, 1980 from Khosta [Ponomarev \& Chumachenko, 2007] are based on misidentification of $S$. wunderlichi (material examined).

DISTRIBUTION. West Caucasus [personal data].

Sintula corniger (Blackwall, 1856)

MATERIAL. 2 우 (VAG) [39].

DISTRIBUTION. West Palaearctic boreo-nemoral range [Mikhailov, 1997; Helsdingen, 2010; Platnick, 2010].

Stemonyphantes agnatus Tanasevitch, 1990*

RECORDS. Gudauta Distr.: Myussera Reserve [Tanasevitch, 1987 - sub S. abantensis Wunderlich, 1978]; Sukhum Distr.: Kelasur [Tanasevitch, 1990; Otto \& Dietzold, 2006].
MATERIAL. $1 O^{7}$ (YMC) [06].

DISTRIBUTION. Crimea and Caucasus [Mikhailov, 1997; Kovblyuk, 2004b; Platnick, 2010; personal data].

Styloctetor romanus (O. Pickard-Cambridge, 1872)

MATERIAL. 1 (YMC) [11].

DISTRIBUTION. Trans-Palaearctic hypoarctonemoral range [Mikhailov, 1997; Marusik et al., 2000; Helsdingen, 2010; Platnick, 2010].

\section{Tenuiphantes mengei (Kulczyński, 1887)}

RECORDS. Gagra Distr.: Pitsunda, Bzyb River Valley; Gudauta Distr.: Myussera Reserve and Ritsa Lake; Sukhum Distr.: Kelasur [Tanasevitch, 1987; Tanasevitch, 1990; Otto \& Dietzold, 2006].

MATERIAL. $30^{7} \sigma^{7}, 4$ Oᄋ (YMC) [06]; 1 (YMC) [07]; 1 우 (YMC) [19]; 1 ㅇ (YMC) [21]; 1 ㅇ (YMC) [24]; 5 o $\sigma^{7}, 14$ 우우 (VAG) [36]; $4 \bigcirc^{7} \sigma^{7}, 8$ क $ᄋ$ (VAG) [39]; 4 우 (VAG) [40]; 1 ㅇ (VAG) [41]; 1 O $^{7}, 6$ 우 (VAG) [45]; 1 + (VAG) [47]; 1 ㅇ (VAG) [54]; $5 \bigcirc^{7} \sigma^{2}, 18$ 우 (VAG) [55]; $10 \bigcirc^{7} \sigma^{7}, 27$ 우 (VAG) [56].

DISTRIBUTION. Trans-Palaearctic boreo-nemoral range [Mikhailov, 1997; Helsdingen, 2010; Platnick, 2010].

Tenuiphantes morosus (Tanasevitch, 1987)

MATERIAL. $2 O^{7} \sigma^{7}$ (VAG) [41].

DISTRIBUTION. Caucasus [Mikhailov, 1997; Helsdingen, 2010; Platnick, 2010].

\section{Tenuiphantes perseus (Helsdingen, 1977)}

RECORDS. Gagra Distr.: Pitsunda; Gudauta Distr.: Ritsa Lake; Sukhum [Tanasevitch, 2009].

DISTRIBUTION. Caucasus and Iran [Tanasevitch, 2009].

Tenuiphantes tenuis (Blackwall, 1852)

MATERIAL. 1 (YMC) [12].

DISTRIBUTION. West and Central Palaearctic boreo-nemoral range [Mikhailov, 1997; Helsdingen, 2010; Platnick, 2010].

Trematocephalus cristatus (Wider, 1834)

MATERIAL. $1+$ (VAG) [36]; 2 + (VAG) [39]; 2 +9 (VAG) [45]; 5 우 (VAG) [55]; 2 ㅇ (VAG) [56].

DISTRIBUTION. Trans-Palaearctic nemoral range [Mikhailov, 1997; Helsdingen, 2010; Platnick, 2010].

Trichoncus affinis Kulczyński, 1894

MATERIAL. 1 (VAG) [55].

DISTRIBUTION. West Palaearctic nemoral range [Mikhailov, 1997; Helsdingen, 2010; Platnick, 2010].

Troglohyphantes charitonovi Tanasevitch, 1987

MATERIAL. $1 \sigma^{7}, 2$ + + (VAG) [54]. 
DISTRIBUTION. Caucasus [Mikhailov, 1997; Platnick, 2010].

Troglohyphantes deelemanae Tanasevitch, 1986*

RECORDS. Gudauta Distr.: Plateau Achibakh (15 km SE of Lake Ritza): caves [Tanasevitch, 1986, 1990; Otto \& Dietzold, 2006].

MATERIAL. 1 ( $(\mathrm{VAG})[\mathbf{4 1}] ; 3$ $+\circ$ (VAG) [55].

DISTRIBUTION. Caucasus [Mikhailov, 1997; Platnick, 2010].

Walckenaeria alticeps (Denis, 1952)

MATERIAL. 1 ( $(\mathrm{VAG})[41]$

DISTRIBUTION. West Palaearctic nemoral range [Mikhailov, 1997; Helsdingen, 2010; Platnick, 2010].

Walckenaeria antica (Wider, 1834) [55].

MATERIAL. $1 \sigma^{7}$ (YMC) [08]; 19 (VAG) [36]; 19 (VAG)

DISTRIBUTION. West and Central Palaearctic nemoral range: east to Yenisei River [Mikhailov, 1997; Marusik et al., 2000; Helsdingen, 2010; Platnick, 2010]. 1878)

Walckenaeria atrotibialis (O. Pickard-Cambridge, [54].

MATERIAL. 1 ( $\mathrm{VAG})[\mathbf{3 2}] ; 1$ (VAG) $[$ 41]; 1 (VAG)

DISTRIBUTION. Circum-Holarctic boreo-nemoral range [Mikhailov, 1997; Helsdingen, 2010; Platnick, 2010].

Walckenaeria vigilax (Blackwall, 1853)

RECORDS. Niszhnaya Yashtukha, Sukhum [Spassky, 1937 - sub Cornicularia; Tanasevitch, 1990; Otto \& Dietzold, 2006].

DISTRIBUTION. Circum-Holarctic boreo-nemoral range [Mikhailov, 1997; Helsdingen, 2010; Platnick, 2010].

LIOCRANIDAE (1 genus and 1 species)

Sagana rutilans Thorell, 1875

MATERIAL. 1 \% (TNU-2639/2) [39].

DISTRIBUTION. West Palaearctic: Europe and Caucasus: from France east to Caucasus and from Poland south to Greece [Mikhailov, 1997; Helsdingen, 2010; Platnick, 2010].

LYCOSIDAE (9 genera and 29 species)

Alopecosa accentuata (Latreille, 1817)

MATERIAL. 1 + (CP) [53].

DISTRIBUTION. Trans-Palaearctic nemoral range [Mikhailov, 1997; Helsdingen, 2010; Platnick, 2010].
«Alopecosa» charitonovi Mcheidze, 1997

Fig. 1.

RECORDS. Gudauta Distr.: Avadkhara [Mcheidze, 1997 — sub Alopecosa; Otto \& Dietzold, 2006].

MATERIAL. $3 \mathrm{O}^{7} \mathrm{O}^{7}, 5$ 우 (TNU-2652/1) [54].

NOTE. This is a poorly known species. Its re-description will be published in a separate paper.

DISTRIBUTION. All Caucasus Major [personal data].

Alopecosa pulverulenta (Clerck, 1757)

MATERIAL. 1 ( $(\mathrm{CP}-25.11 .2 / 24)$ [01]; $18 \mathrm{O}^{7} \mathrm{O}^{7}, 11$ 우 (TNU2641/15) [41]; 1 + (TNU-2652/38) [54].

DISTRIBUTION. Trans-Palaearctic boreo-nemoral range [Mikhailov, 1997; Helsdingen, 2010; Platnick, 2010].

Arctosa leopardus (Sundevall, 1833)

RECORDS. Sukhum [Mcheidze, 1997; Otto \& Dietzold, 2006].

MATERIAL. 1 ( $(\mathrm{CP})[6 \mathbf{6 3}]$.

DISTRIBUTION. West and Central Palaearctic nemoral range [Mikhailov, 1997; Helsdingen, 2010; Platnick, 2010].

Arctosa tbilisiensis Mcheidze, 1946

MATERIAL, $1 \sigma^{7}, 1$ (TNU-2636/14) [36]; $1 \sigma^{7}$ (TNU-2639/ 33) [39]; 4 O $^{\top} \sigma^{\top}, 1$, 1 (TNU-2651/60) [55]; 4 O $^{7} O^{T}$ (TNU-2653/39) [56].

DISTRIBUTION. Balkan-Caucasian range: Greece, Bulgaria, Krasnodar Area, Daghestan, Abkhazia, Georgia, Azerbaijan and Iran [Ovtsharenko, 1979; Mikhailov, 1997; Ponomarev \& Alieva, 2010; Helsdingen, 2010; Platnick, 2010; personal data].

Aulonia albimana (Walckenaer, 1805)

MATERIAL. 3 우 (CP-25.19.1/2) [29]; $1 \bigcirc^{7}$ (TNU-2639/28) [39]; $1 \mathrm{O}^{\top}$ (TNU-2640/3) [40]; 9 우 (TNU-2653/37) [56]; 1 ㅇ (CP) [62].

DISTRIBUTION. West Palaearctic nemoral range [Mikhailov, 1997; Helsdingen, 2010; Platnick, 2010].

Geolycosa vultuosa (C.L. Koch, 1838)

RECORDS. Sukhum [Mcheidze, 1964, 1997 sub Lycosa; Otto \& Dietzold, 2006].

DISTRIBUTION. Southeastern Europe to Central Asia: from Sardinia east to Middle Asian Mts, and from the Russian Plain south to Greece [Mikhailov, 1997; Helsdingen, 2010; Platnick, 2010].

Hogna radiata (Latreille, 1817)

RECORDS. Gagra; Sukhum [Spassky, 1937 and Mcheidze, 1964, 1997 — sub Lycosa; Otto \& Dietzold, 2006]. 


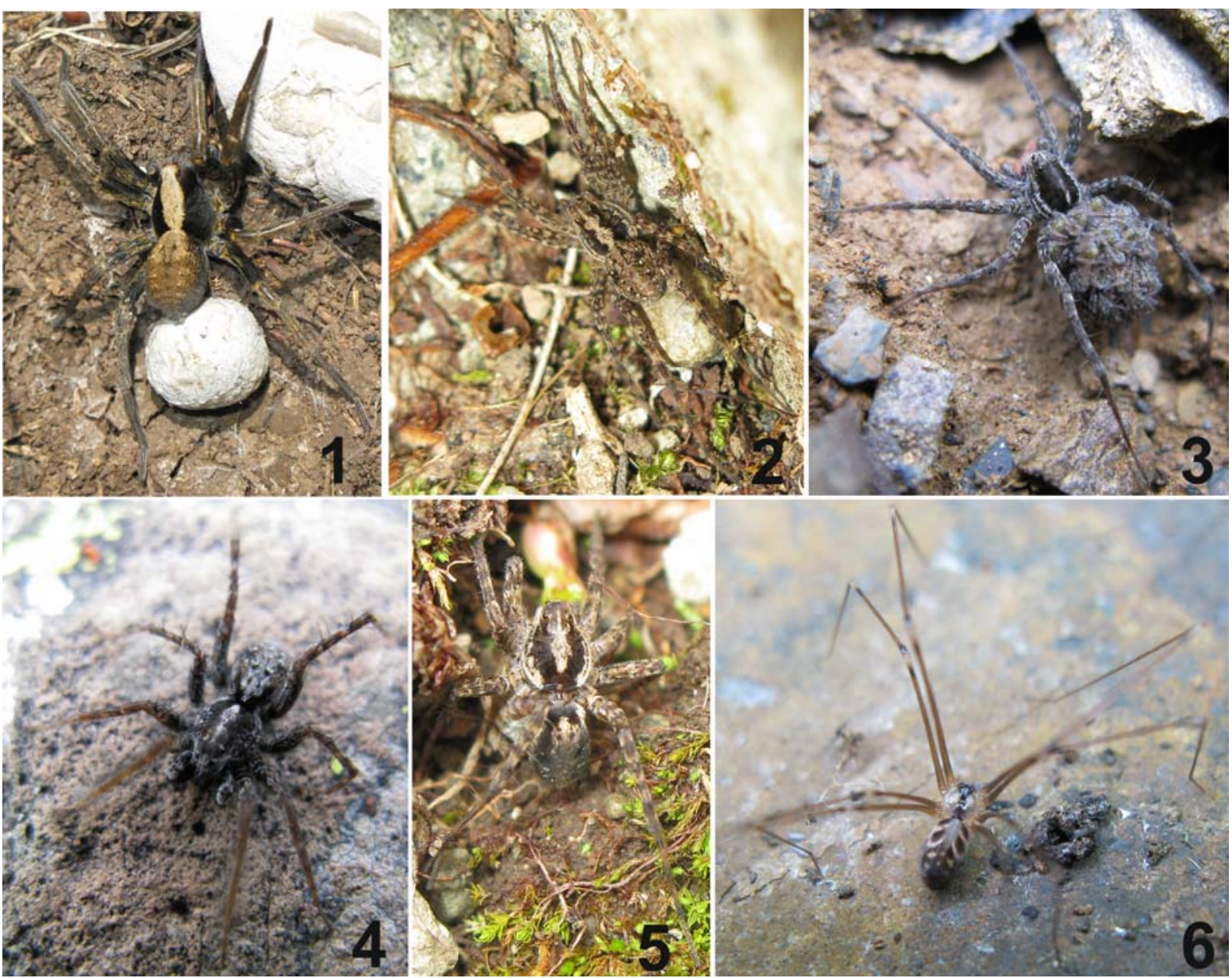

Figs 1-6. Habitus: 1 - female of «Alopecosa» charitonovi with egg sac; 2 - female of Pardosa abagensis with egg sac; 3 - female of Pardosa buchari with spiderlings; 4 - male of Pardosa incerta; 5 - female of Pardosa incerta; 6 - male of Hoplopholcus longipes.

Рис. 1-6. Внешний вид при жизни: 1 - самка «Alopecosa» charitonovi с коконом; 2 - самка Pardosa abagensis с коконом; 3 самка Pardosa buchari с паучками; 4 - самец Pardosa incerta; 5 - самка Pardosa incerta; 6 - самец Hoplopholcus longipes.

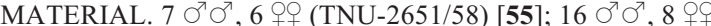
(TNU-2653/34) [56]

DISTRIBUTION. West and Central Palaearctic nemoral-subtropical range and Central Africa [Mikhailov, 1997; Helsdingen, 2010; Platnick, 2010].

Pardosa abagensis Ovtsharenko, 1979

Fig. 2.

MATERIAL. $20^{7} \sigma^{7}, 3$ 오 (TNU-2641/17) [41].

DISTRIBUTION. West Caucasus [Ovtsharenko, 1979; Mikhailov, 1997; Platnick, 2010; personal data].

Pardosa buchari Ovtsharenko, 1979

Fig. 3.

MATERIAL. $25 \sigma^{7} \sigma^{7}, 63$ 우 (TNU-2652/37) [54].

DISTRIBUTION. Crimea and West Caucasus [Mikhailov, 1997; Kovblyuk, 2004c; Helsdingen, 2010; Platnick, 2010].

\section{Pardosa caucasica Ovtsharenko, 1979} [54].

MATERIAL. 1 ( (ZMMU) [09]; $20^{7} \sigma^{\top}, 4$ ㅇ (TNU-2652/36)

DISTRIBUTION. Caucasus [Mikhailov, 1997; Helsdingen, 2010; Platnick, 2010].

Pardosa colchica Mcheidze, 1946

RECORDS. Gentsvishi (E of Sukhum) [Zyuzin \& Logunov, 2000; Otto \& Dietzold, 2006].

MATERIAL. $60^{7} O^{7}, 10$ ㅇ (TNU-2653/41) [56]

DISTRIBUTION. Caucasus [Mikhailov, 1997; Helsdingen, 2010; Platnick, 2010].

Pardosa hortensis (Thorell, 1872)

RECORDS. Sukhum [Mcheidze, 1964; Otto \& Dietzold, 2006]

MATERIAL. $14 \sigma^{7} \sigma^{7}, 32 \circ \bigcirc$ (TNU-2651/64) [55]; $5 \sigma^{7} \sigma^{7}, 59$ 우우 (TNU-2653/43) [56]. 
DISTRIBUTION. West and Central Palaearctic nemoral range [Mikhailov, 1997; Helsdingen, 2010; Platnick, 2010].

Pardosa incerta Nosek, 1905

Figs $4-5$.

MATERIAL. $960^{7} \sigma^{7}, 148$ 우 (TNU-2641/16) [41].

DISTRIBUTION. Bulgaria, Turkey and Caucasus [Zyuzin \& Ovtsharenko, 1979; Mikhailov, 1997; Helsdingen, 2010; Platnick, 2010].

Pardosa lugubris (Walckenaer, 1802)

MATERIAL. 4 오 (TNU-2651/66) [55].

DISTRIBUTION. West-Palaearctic nemoral range [Mikhailov, 1997; Helsdingen, 2010; Platnick, 2010, personal data].

? Pardosa monticola (Clerck, 1757)

RECORDS. Ritsa Lake, Sukhum [Mcheidze, 1964, 1997; Otto \& Dietzold, 2006].

DISTRIBUTION. West and Central Palaearctic nemoral range [Mikhailov, 1997; Helsdingen, 2010; Platnick, 2010].

Pardosa nebulosa orientalis (Kroneberg, 1875)

RECORDS. Sukhum [Mcheidze, 1964, 1997 sub $P$. orientalis; Otto \& Dietzold, 2006].

MATERIAL. $1 \bigcirc^{\top}, 2$ ㅇ (TNU-2653/36) [56]; 19 (TNU-2655/ 1) $[\mathbf{5 8}] ; 1 \sigma^{7}(\mathrm{CP})[\mathbf{6 3}]$.

DISTRIBUTION. Central-Palaearctic nemoral range [Mikhailov, 1997; Platnick, 2010].

Pardosa pontica (Thorell, 1875)

MATERIAL. 6 O $^{7} \sigma^{7}$ (TNU-2636/18) [36]; $104 \bigcirc^{7} \sigma^{7}, 87$ 우 (TNU-2651/63) [55]; 4 ○ $\bigcirc^{7}, 4$ 우 (TNU-2653/40) [56].

DISTRIBUTION. From Bulgaria east to West Turkmenistan [Mikhailov, 1997; Helsdingen, 2010; Platnick, 2010].

Pardosa prativaga (L. Koch, 1870) [45]

MATERIAL. 2 오 (TNU-2639/31) [39]; 4 오 (TNU-2644/30)

DISTRIBUTION. Trans-Palaearctic nemoral range [Mikhailov, 1997; Helsdingen, 2010; Platnick, 2010].

Pardosa proxima (C.L. Koch, 1847)

MATERIAL. $1 \bigcirc^{7}, 2$ 우 (TNU-2653/43) [56]

DISTRIBUTION. Trans-Palaearctic nemoral range [Mikhailov, 1997; Helsdingen, 2010; Platnick, 2010].

Pirata hurkai Buchar, 1966*

RECORDS. Gudauta Distr.: Ritsa Lake [Buchar, 1966; Mcheidze, 1997; Otto \& Dietzold, 2006].
MATERIAL. $12 \sigma^{7} \sigma^{7}, 33$ Oᄋ (TNU-2636/16) [36]; $3 \sigma^{\top} \sigma^{\top}, 9$ (TNU-2639/30) [39]; 1 ऊ (CP) [60].

DISTRIBUTION. Crimea and West Caucasus [Mikhailov, 1997; Kovblyuk, 2002, 2004c; Platnick, 2010].

Pirata hygrophilus Thorell, 1872

MATERIAL. 3 우 (TNU-2636/15) [36]; 1 ○, 7 우 (TNU2639/29) [39]; 9 우 (TNU-2653/44) [56].

DISTRIBUTION. Palaearctic boreo-nemoral range [Mikhailov, 1997; Helsdingen, 2010; Platnick, 2010].

? Pirata insularis Emerton, 1885

RECORDS. Kelasur [Mcheidze, 1997 - sub $P$. picollo; Otto \& Dietzold, 2006].

DISTRIBUTION. Holarctic boreo-nemoral range [Mikhailov, 1997; Helsdingen, 2010; Platnick, 2010].

Trochosa cachetiensis Mcheidze, 1997

MATERIAL. $4 \sigma^{\top} \sigma^{7}$ (CP) [61].

NOTE. It is poorly known species. It will be redescribed in a separate paper.

DISTRIBUTION. West Caucasus: Abkhazia and Georgia [Mcheidze, 1997; Otto \& Dietzold, 2006].

\section{? Trochosa hispanica Simon, 1870}

MATERIAL. 2 우 (CP) [53]; $1 \sigma^{7}$ (CP) [61].

NOTE: The identification of this species is preliminary. The species differs from Trochosa ruricola (De Geer, 1778) by its smaller size. It is similar to $T$. ruricola by the presence of a tooth on the cheliceral claw.

DISTRIBUTION. Mediterranean and Caucasus: from Portugal east to Azerbaijan, and from France south to Crete [Helsdingen, 2010; Platnick, 2010].

Trochosa robusta (Simon, 1876)

MATERIAL. $10^{7}, 8$ 8 (TNU-2639/35) [39].

DISTRIBUTION. Trans-Palaearctic nemoral range [Mikhailov, 1997; Helsdingen, 2010; Platnick, 2010].

Trochosa ruricola (De Geer, 1778)

RECORDS. Sukhum; Tsebelda [Schmidt, 1895 sub Lycosa; Simon, 1899; Verzhbitsky, 1902; Kharitonov, 1932; Mcheidze, 1997; Otto \& Dietzold, 2006]. MATERIAL. $10^{7}, 5$ ㅇ (TNU-2639/34) [39].

DISTRIBUTION. Circum-Holarctic nemoral range, Bermuda [Mikhailov, 1997; Helsdingen, 2010; Platnick, 2010].

Trochosa terricola Thorell, 1856

MATERIAL. $1 \bigcirc^{7}$ (ZMMU) [04]

DISTRIBUTION. Circum-Holarctic nemoral range [Mikhailov, 1997; Helsdingen, 2010; Platnick, 2010]. 
Xerolycosa nemoralis (Westring, 1861)

MATERIAL. $3 \sigma^{x} \sigma^{7}, 2$ ㅇ (TNU-2640/4) [40].

DISTRIBUTION. Trans-Palaearctic nemoral range [Mikhailov, 1997; Helsdingen, 2010; Platnick, 2010].

MIMETIDAE (2 genera and 3 species)

Ero aphana (Walckenaer, 1802)

MATERIAL. $1 \sigma^{7}$ (TNU-2636/63) [36]; 1 (TNU-2651/6) [55]; 1 ㅇ (TNU-2653/62) [56]; 1 ㅇ (CP) [62].

DISTRIBUTION. West and Central Palaearctic nemoral range, introduced in St. Helena, Queensland, Western Australia [Mikhailov, 1997; Helsdingen, 2010; Platnick, 2010].

Ero furcata (Villers, 1789)

MATERIAL. 1 (TNU-2653/63) [56].

DISTRIBUTION. Trans-Palaearctic nemoral range [Mikhailov, 1997; Helsdingen, 2010; Platnick, 2010].

Mimetus laevigatus (Keyserling, 1863)

RECORDS. Sukhum [Mcheidze, 1997; Otto \& Dietzold, 2006].

DISTRIBUTION. Ancient Mediterranean range: from Portugal east to Middle Asian Mts, and from the Russian Plain south to Sicily, Crete, Turkey [Mikhailov, 1997; Helsdingen, 2010; Platnick, 2010].

MYSMENIDAE (1 genus and 1 species)

Mysmenella jobi (Kraus, 1967)

MATERIAL. 1 (YMC) [06]; 1 (TNU-2636/70) [36].

DISTRIBUTION. West Palaearctic nemoral range: from France east to Azerbaijan, and from Poland and Germany south to Italy [Mikhailov, 1997; Helsdingen, 2010; Platnick, 2010]. Records from the Far East (Maritime Prov., Beijing, Sakhalin and Moneron Islands, Korea) probably refer to another species [Marusik \& Guseinov, 2003].

NEMESIIDAE (1 genus and 1 species)

Raveniola pontica (Spassky, 1937)

RECORDS. Gudauta Distr.: Novyi Aphon [Wagner, 1896; Charitonov, 1936; Mikhailov, 1997 - all sub Mygale caucasica Wagner, 1896, nomen nudum].

MATERIAL. 1 + (ZMMU) [05]; $10^{7}$ (ZMMU) [09]; $10^{7}$ (CP 45.10.1/4) [33] 1 \& (TNU-2636/47) [36]; $1 O^{7}$ (TNU-2644/2) [45].

DISTRIBUTION. West Caucasus from Anapa south to Batum [Kovblyuk \& Ponomarev, 2008].

NESTICIDAE (2 genera and 4 species)

Aituaria pontica (Spassky, 1932)

RECORDS. Gudauta Distr., Tarkily Cave [Charitonov, 1947 — sub Nesticus; Dzhanashvili, 1965 - sub Nesticus; Mcheidze, 1997; Otto \& Dietzold, 2006].

DISTRIBUTION. West Caucasus [Mikhailov, 1997; Platnick, 2010].

? Carpathonesticus borutzkyi (Reimoser, 1930)

RECORDS. Absent. All records from Abkhazia actually must be referred to Georgia sensu stricto: "RionHohle bei Kutais" [Reimoser, 1930] and cave near Tshaltubo [Charitonov, 1941; Dzhanashvili, 1965; Nadolny \& Kovblyuk, 2006].

DISTRIBUTION. Crimea, Caucasus, Turkey [Mikhailov, 1997; Platnick, 2010].

Carpathonesticus caucasicus (Charitonov, 1947)*

RECORDS. Gudauta Distr.: Anysh Grotte, Tchlakhe Cave, Agumava Cave [Charitonov, 1947; Dzhanashvili, 1965; Mcheidze, 1964, 1997; Otto \& Dietzold, 2006].

NOTE. We collected some Carpathonesticus specimens, but their identification is problematic before revision.

DISTRIBUTION. West Caucasus [Mikhailov, 1997; Platnick, 2010].

Carpathonesticus zaitzevi (Charitonov, 1939)*

RECORDS. Akhali Atoni, Sukhum Distr.: Adzaba Cave, Kelasur Cave, cave near Mikhailovskoe Vil., cave „Rodnik“, Aphon Cave, Verkhne-Andreevskaya Cave, Akhalsheni, Shroma; Gulrypsh Distr.: cave in Tsebeldy; Verkhne-Shakura cave; Ochamchyra Distr.: Golova Atapa Cave [Charitonov, 1936, 1939, 1941, 1947; Pichka, 1965; Dzhanashvilli, 1965 — all sub Nesticus; Mcheidze, 1964, 1997; Otto \& Dietzold, 2006].

NOTE. We collected some additional Carpathonesticus specimens, but their identification is problematic before revision.

DISTRIBUTION. West Caucasus [Mikhailov, 1997; Platnick, 2010].

OECOBIIDAE ( 1 genus and 1 species)

Oecobius navus Blackwall, 1859

RECORDS. Sukhum [Charitonov, 1936; Spassky, 1937; Mcheidze, 1997; Otto \& Dietzold, 2006 - all sub Oecobius annulipes (Lucas, 1846)].

MATERIAL. 1 (TNU-2638/7) [38].

DISTRIBUTION. Cosmopolitan [Mikhailov, 1997; Helsdingen, 2010; Platnick, 2010].

OONOPIDAE (1 genus and 1 species)

Oonops domesticus (Dalmas, 1916)

RECORDS. Sukhum [Marusik, 2005; Otto \& Dietzold, 2006]. 
DISTRIBUTION. West Palaearctic nemoral range [Mikhailov, 1997; Helsdingen, 2010; Platnick, 2010].

OXYOPIDAE (1 genus and 1 species)

Oxyopes lineatus Latreille, 1806

RECORDS. Sukhum; Gulripsh Distr.: Tsebelda [Simon, 1899; Verzhbitsky, 1902; Spassky, 1937; Mcheidze, 1997; Otto \& Dietzold, 2006].

MATERIAL. 2 우 (CP-16.10.1/7) [02]; $2 \sigma^{7} \sigma^{7}$ (ZMMU) [05] 1 오 (TNU-2636/26) [36]; 1 ○ (TNU-2639/8) [39]; 1 ㅇ (TNU2651/2) [55]; 1 ○' (TNU-2653/5) [56]

DISTRIBUTION. West and Central Palaearctic nemoral range [Mikhailov, 1997; Platnick, 2010].

PHILODROMIDAE (1 genus and 3 species)

Philodromus cespitum (Walckenaer, 1802)

MATERIAL. $1+$ (CP-36.10.15/4) [01].

DISTRIBUTION. Circum-Holarctic polyzonal range [Mikhailov, 1997; Helsdingen, 2010; Platnick, 2010].

Philodromus collinus C.L. Koch, 1835

MATERIAL. 3 우 (TNU-2652/14) [54]

DISTRIBUTION. West and Central Palaearctic nemoral range [Mikhailov, 1997; Platnick, 2010].

Philodromus poecilus (Thorell, 1872)

MATERIAL. 1 \% (CP-36.10.4/9) [35].

DISTRIBUTION. Trans-Palaearctic nemoral range [Mikhailov, 1997; Helsdingen, 2010; Platnick, 2010].

PHOLCIDAE (2 genera and 2 species $)$

Hoplopholcus longipes (Spassky, 1934) Figs 6-34.

Holocnemus l. Spassky, 1934: 366, f. 4-7 ( ( H. l: Brignoli, 1978: 493, f. 50-56 ( $\sigma^{\top}$ )

Holocnemus l.: Mcheidze, 1997: 65, f. 56-57 ().

RECORDS. Gudauta, Verkhne-Mzymta Cave, Lykhny Cave, Shiroko-pokosskaya Cave, Agumava Cave [Charitonov, 1947; Mcheidze, 1964; Otto \& Dietzold, 2006].

MATERIAL. $1 \bigcirc^{7}, 2$ ㅇ (TNU-2639/57) [39]; 19 (TNU-2644/

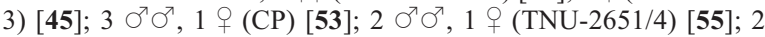
$\sigma^{7} \sigma^{7}, 1$ (TNU-2653/24) [56].

DESCRIPTION. Measurements $\left(\sigma^{7} / 7\right)$ : total length 5.2 / 5.4; carapace 2.1 / 2.1 long, 1.9 / 2.0 wide. Length of leg segments (male/female):

\begin{tabular}{|l|c|c|c|c|c|}
\hline Leg & Femur & Patella & Tibia & Metatarsus & Tarsus \\
\hline I & $10.3 / 12.0$ & $0.8 / 0.9$ & $10.3 / 12.4$ & $14.4 / 16.9$ & $2.9 / 3.2$ \\
\hline II & $8.0 / 9.3$ & $0.7 / 0.9$ & $7.2 / 8.9$ & $9.3 / 12.2$ & $1.6 / 2.4$ \\
\hline III & $6.8 / 7.8$ & $0.7 / 0.8$ & $5.9 / 6.9$ & $7.8 / 9.0$ & $1.7 / 1.8$ \\
\hline IV & $8.0 / 9.1$ & $0.8 / 0.9$ & $7.2 / 8.0$ & $9.3 / 11.1$ & $1.7 / 1.8$ \\
\hline
\end{tabular}

Males have 22-26 fine spines on Femur I venter $(n=3)$.
DISTRIBUTION. Turkey and West Caucasus [Mikhailov, 1997; Platnick, 2010].

Pholcus phalangioides (Fuesslin, 1775)

RECORDS. Akhali Atoni, Sukhum, Tsebelda [Spassky, 1937; Mcheidze, 1964, 1997 — sub Pholcus phalangoides; Otto \& Dietzold, 2006].

MATERIAL. 7 Oᄋ (YMC) [15]; 2 오 (TNU-2639/56) [39]; 1 $\mathrm{O}^{\top}$ (CP-35.10.2/13) [50]; 1 ㅇ (CP-35.10.2/12) [51]; 6 우 (CP) [53]; $2 \bigcirc^{7} \sigma^{7}, 2$ 우 (TNU-2651/10) [55]; 3 우 (CP) [62].

DISTRIBUTION. Cosmopolitan [Platnick, 2010].

PISAURIDAE (1 genus and 2 species)

? Pisaura mirabilis (Clerck, 1757)

Figs 35-36, 39-40, 42.

RECORDS. Sukhum [Spassky, 1937].

NOTE. Probably S.A. Spassky misidentified P. novicia as P. mirabilis (see comments for the following species).

DISTRIBUTION. West and Central Palaearctic nemoral range [Mikhailov, 1997; Platnick, 2010].

? Pisaura novicia (L. Koch, 1878)

Figs 37-38, 41, 43.

Ocyale n. L. Koch, 1878: 54, pl. 2, f. 3 ( $\left.\sigma^{7}+\right)$.

P. n. : Simon, 1898: 289

P. n. : Blandin, 1976: 922, f. 12 (ㅇ).

P. n. : Brignoli, 1984: 36, f. 3-4 (+).

P. n.: Mcheidze, 1997: 246, f. 536 (ㅇ).

RECORDS. Gagra [Simon, 1899; Verzhbitsky, 1902; Mcheidze, 1997; Otto \& Dietzold, 2006].

MATERIAL. 1 ㅇ (CP-13.10.1/9) [03]; 1 (CP-13.10.1/14) [29]; 4 우 (TNU-2636/27) [36]; 1 क (TNU-2639/1) [39]; 1 ㅇ

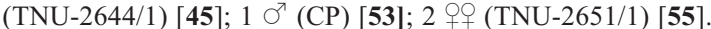

COMPARATIVE MATERIAL. Pisaura mirabilis: UKRAINE: Kyiv Area, Tetiiv Distr., Ros River: 2 +O (TNU), 21.07.2006 and 2.08.2006, A.A. Nadolny.

Pisaura sp.: UKRAINE, CRIMEA: numerous males and females.

NOTES. P.M. Brignoli [1984] mentioned that Pisaura species can be distinguished by epigynes only. Specimens from Crimea have variable shapes of epigynes, although they are more similar to ?P. novicia from Abkhasia than to $P$. mirabilis from continental part of Ukraine. The problem with identification of Crimean Pisaura species will be addressed in the future.

DISTRIBUTION. East Mediterranean: from Italy east to West Caucasus [Mikhailov, 1997; Helsdingen, 2010; Platnick, 2010].

SALTICIDAE (19 genera and 24 species)

Ballus chalybeius (Walckenaer, 1802)

RECORDS. Sukhum [Mcheidze, 1997 referring to Spassky [1937] however, Spassky did not indicate this species for Sukhum; Otto \& Dietzold, 2006]. 

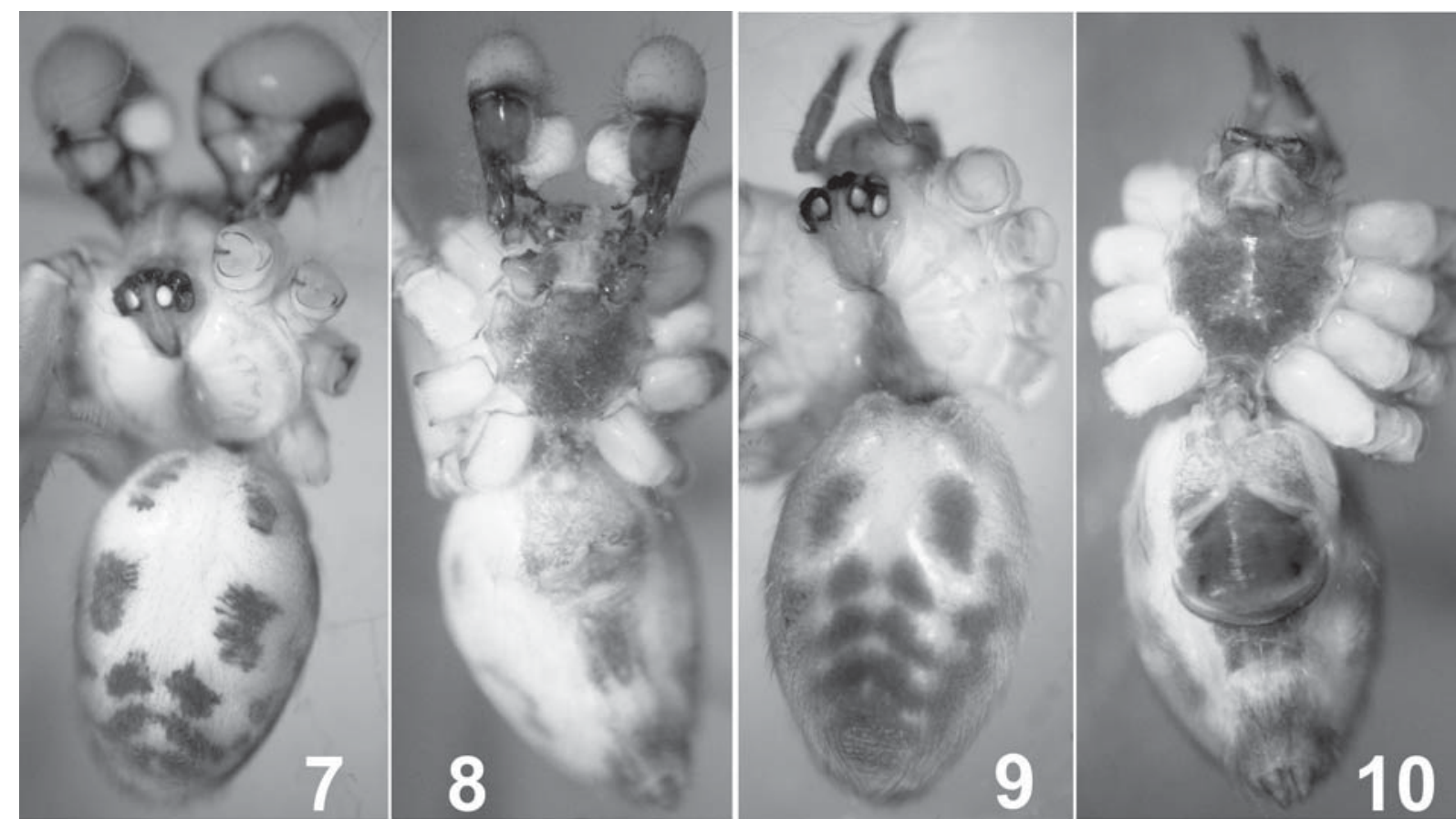

Figs 7-10. Habitus of Hoplopholcus longipes: 7 - male, dorsal view; 8 - male, ventral view; 9 - female, dorsal view; 10 - female, ventral view.

Рис. 7-10. Габитус Hoplopholcus longipes: 7 - самец, дорсально; 8 - самец, вентрально; 9 - самка, дорсально; 10 - самка, вентрально.

DISTRIBUTION. West and Central Palaearctic nemoral range [Mikhailov, 1997; Logunov \& Marusik, 2000; Helsdingen, 2010; Platnick, 2010].

Carrhotus xanthogramma (Latreille, 1819)

RECORDS. Gagra; Sukhum [Mcheidze, 1964, 1997; Logunov \& Guseinov, 2002; Otto \& Dietzold, 2006].

MATERIAL. 1 + (CP-17.31.1/10) [03].

DISTRIBUTION. Trans-Palaearctic nemoral range [Mikhailov, 1997; Logunov \& Marusik, 2000; Helsdingen, 2010; Platnick, 2010].

\section{Chalcoscirtus infimus (Simon, 1868)}

MATERIAL. 1 (TNU-2636/55) [36]; 1 ( $(\mathrm{CP})[62]$.

DISTRIBUTION. West and Central Palaearctic nemoral range [Mikhailov, 1997; Helsdingen, 2010; Platnick, 2010].

\section{Euophrys frontalis (Walckenaer, 1802)}

MATERIAL. 1 \% (YMC) [10]; 3 우 (YMC) [12]; 13 우 (YMC) [24]; $1 \bigcirc^{7}$ (CP-17.20.1/19) [29]; 5 O $^{7} \sigma^{7}, 4$ 우 (TNU-2639/24) [39]; 1 ㅇ (TNU-2644/23) [45]; 7 우 (TNU-2651/44) [55].

DISTRIBUTION. Trans-Palaearctic boreo-nemoral range [Mikhailov, 1997; Logunov \& Marusik, 2000; Helsdingen, 2010; Platnick, 2010].

Evarcha arcuata (Clerck, 1757)

RECORDS. Sukhum [Spassky, 1937; Mcheidze, 1997; Otto \& Dietzold, 2006].
MATERIAL. $6 \sigma^{7} \sigma^{7}, 1+(\mathrm{CP}-17.17 .1 / 30)[02] ; 2 \sigma^{7} \sigma^{7}, 4$ 우 (TNU-2636/54) [36]; $15 \mathrm{O}^{7} \mathrm{O}^{7}, 17$ 우 (TNU-2639/19) [39]; $4 \mathrm{O}^{7} \mathrm{O}^{7}$, 7 아 (TNU-2644/19) [45]; 1 ㅇ (TNU-2647/6) [48]; 3 ○ $^{7} \sigma^{7}, 2$ 우 (TNU-2651/37) [55]; $2 \bigcirc^{\top} \bigcirc^{7}, 4$ 우 (TNU-2653/49) [56].

DISTRIBUTION. Trans-Palaearctic boreo-nemoral range [Mikhailov, 1997; Logunov \& Marusik, 2000; Helsdingen, 2010; Platnick, 2010].

\section{Heliophanus auratus C.L. Koch, 1835}

RECORDS. Sukhum [Spassky, 1937 and Mcheidze, 1997 - sub H. nigriceps Kulcz.; Otto \& Dietzold, 2006].

DISTRIBUTION. West and Central Palaearctic nemoral range, east to Lena River [Mikhailov, 1997; Helsdingen, 2010; Platnick, 2010].

Heliophanus cupreus (Walckenaer, 1802)

RECORDS. Sukhum [Spassky, 1937; Mcheidze, 1997; Otto \& Dietzold, 2006], Gagra Distr.: Pitsunda, pine forest (sweeping on Carex sp.) [Logunov \& Guseinov, 2002].

MATERIAL. $1 \sigma^{7}$ (CP-17.21.1/18) [02]; $4 \sigma^{7} \sigma^{7}, 1$ (TNU-2639/ 23) [39]; $1 \bigcirc^{\top}$ (TNU-2643/3) [44]; $2 \mathrm{O}^{\top} \sigma^{\top}, 3$ +क (TNU-2644/20) [45].

DISTRIBUTION. West and Central Palaearctic nemoral range, east to Yenisei River [Mikhailov, 1997; Logunov \& Marusik, 2000; Helsdingen, 2010; Platnick, 2010].

Macaroeris nidicolens (Walckenaer, 1802)

RECORDS. Akhali Atoni; Sukhum [Mcheidze, 1964, 1997 — sub Dendryphantes; Otto \& Dietzold, 2006]. 

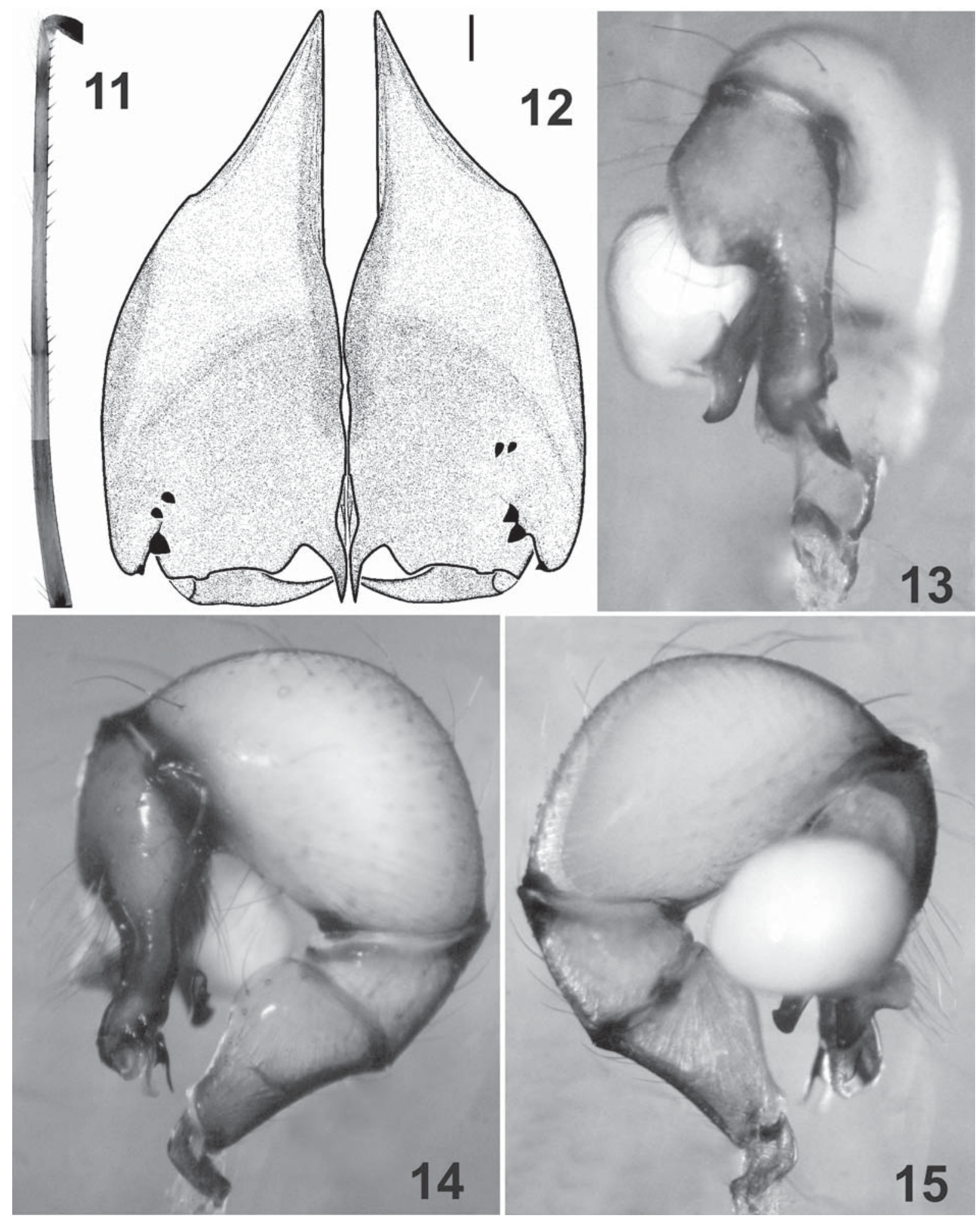

Figs 11-15. Male of Hoplopholcus longipes: 11 - femur I; 12 - chelicerae, frontal view; 13 - palp, dorsal view; 14 - palp, retrolateral view; 15 - palp, prolateral view.

Рис. 11-15. Самец Hoplopholcus longipes: 11 - бедро I; 12 - хелицеры, фронтально; 13 - пальпа, дорсально; 14 - пальпа, ретролатерально; 15 - пальпа, пролатерально. 


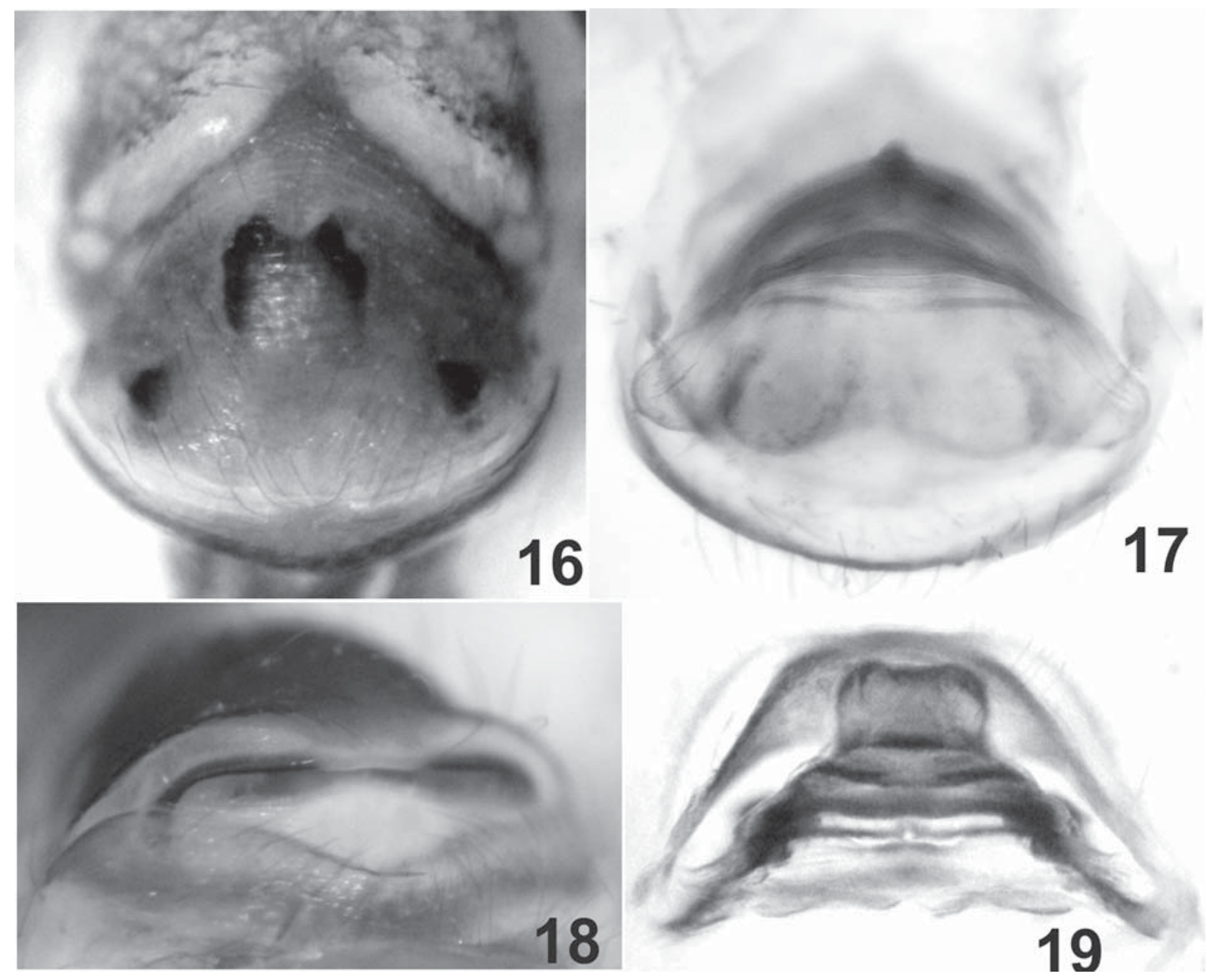

Figs 16-19. Epigyne of Hoplopholcus longipes: 16 - ventral view; 17 - dorsal view; 18 - caudal view; 19 - frontal view. Рис. 16-19. Эпигина Hoplopholcus longipes: 16 - вентрально; 17 - дорсально; 18 - сзади; 19 - спереди.
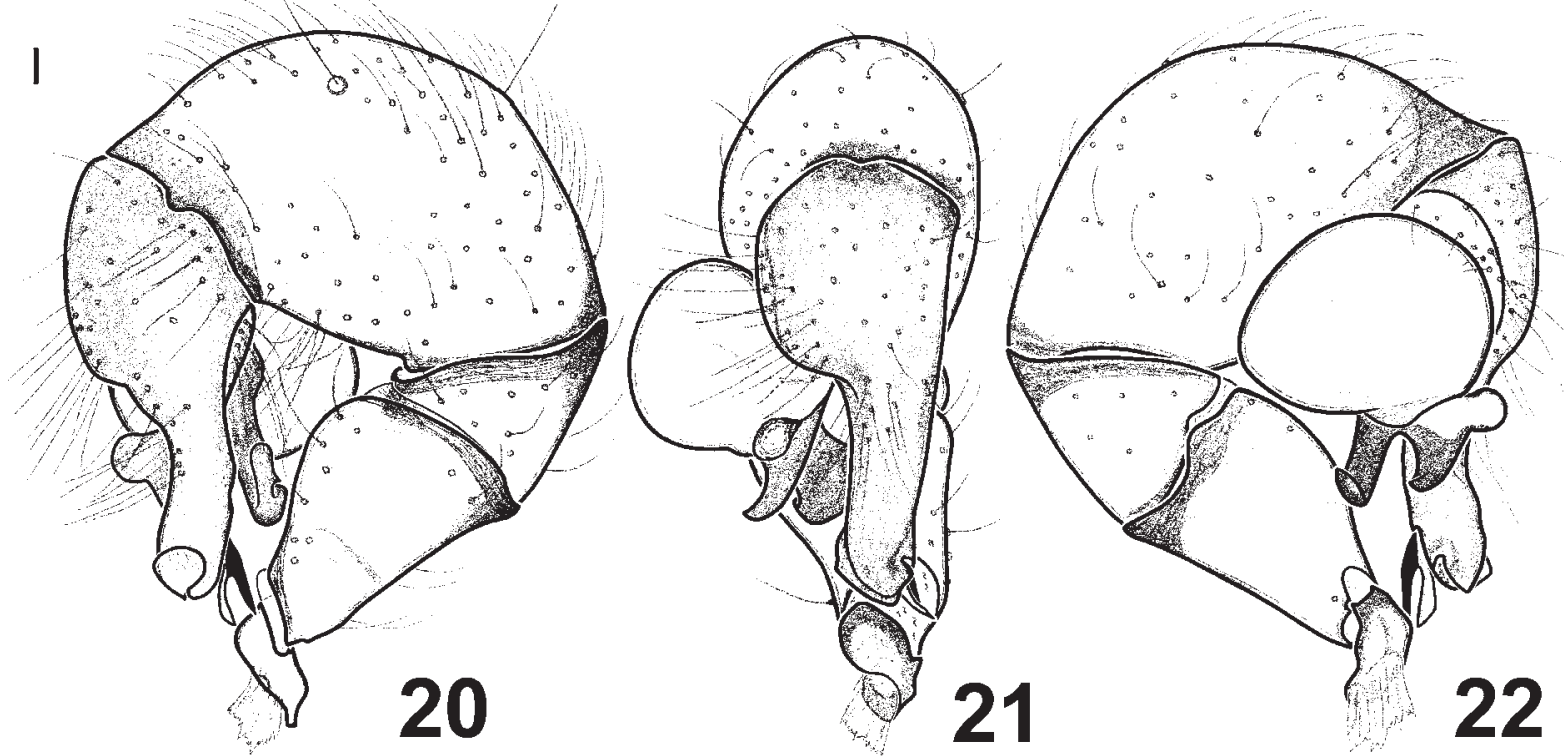

Figs 20-22. Male palp of Hoplopholcus longipes: 20 - retrolateral view; 21 - dorsal view; 22 - prolateral view.

Рис. 20-22. Пальпа самца Hoplopholcus longipes: 20 - ретролатерально; 21 - дорсально; 22 - пролатерально. 

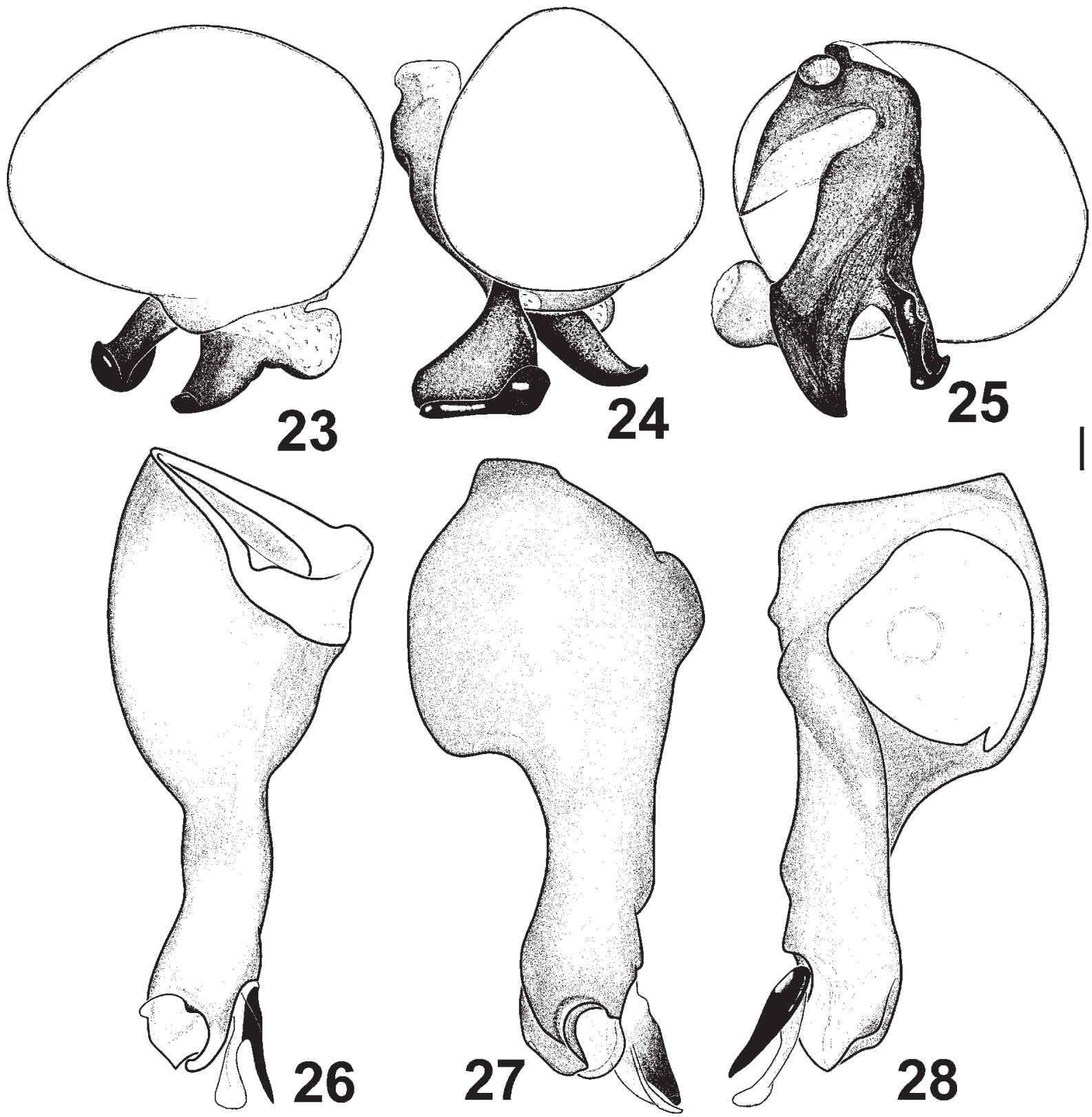

Figs 23-28. Detailes of male palp of Hoplopholcus longipes: 23 - bulbus, prolateral view; 24 - bulbus, ventral view; 25 - bulbus, retrolateral view; 26 - apical part of palp, retrolateral view; 27 - apical part of palp, dorsal view; 28 - apical part of palp, prolateral view.

Рис. 23-28. Детали стрения пальпы самца Hoplopholcus longipes: 23 - бульбус, пролатерально; 24 - бульбус, вентрально; 25 бульбус, ретролатерально; 26 - апикальная часть пальпы, ретролатерально; 27 - апикальная часть пальпы, дорсально; 28 апикальная часть пальпы, пролатерально. [55].

MATERIAL. 1 (TNU-2636/53) [36]; 1 (TNU-2651/46)

DISTRIBUTION. West Palaearctic nemoral range [Mikhailov, 1997; Helsdingen, 2010; Platnick, 2010].

\section{Marpissa muscosa (Clerck, 1757)}

MATERIAL. $1 O^{7}$ (YMC) [25]; $1 O^{\top}(\mathrm{CP})[\mathbf{6 0}]$.

DISTRIBUTION. West Palaearctic nemoral range [Mikhailov, 1997; Logunov \& Marusik, 2000; Helsdingen, 2010; Platnick, 2010].

\section{Marpissa nivoyi (Lucas, 1846)}

[56].

MATERIAL. 1 (TNU-2651/39) [55]; 5 우 (TNU-2653/47) DISTRIBUTION. West and Central Palaearctic nemoral range [Mikhailov, 1997; Helsdingen, 2010; Platnick, 2010].

Mendoza canestrinii (Ninni, 1868)

RECORDS. Gagra [Simon, 1899 and Verzhbitsky, 

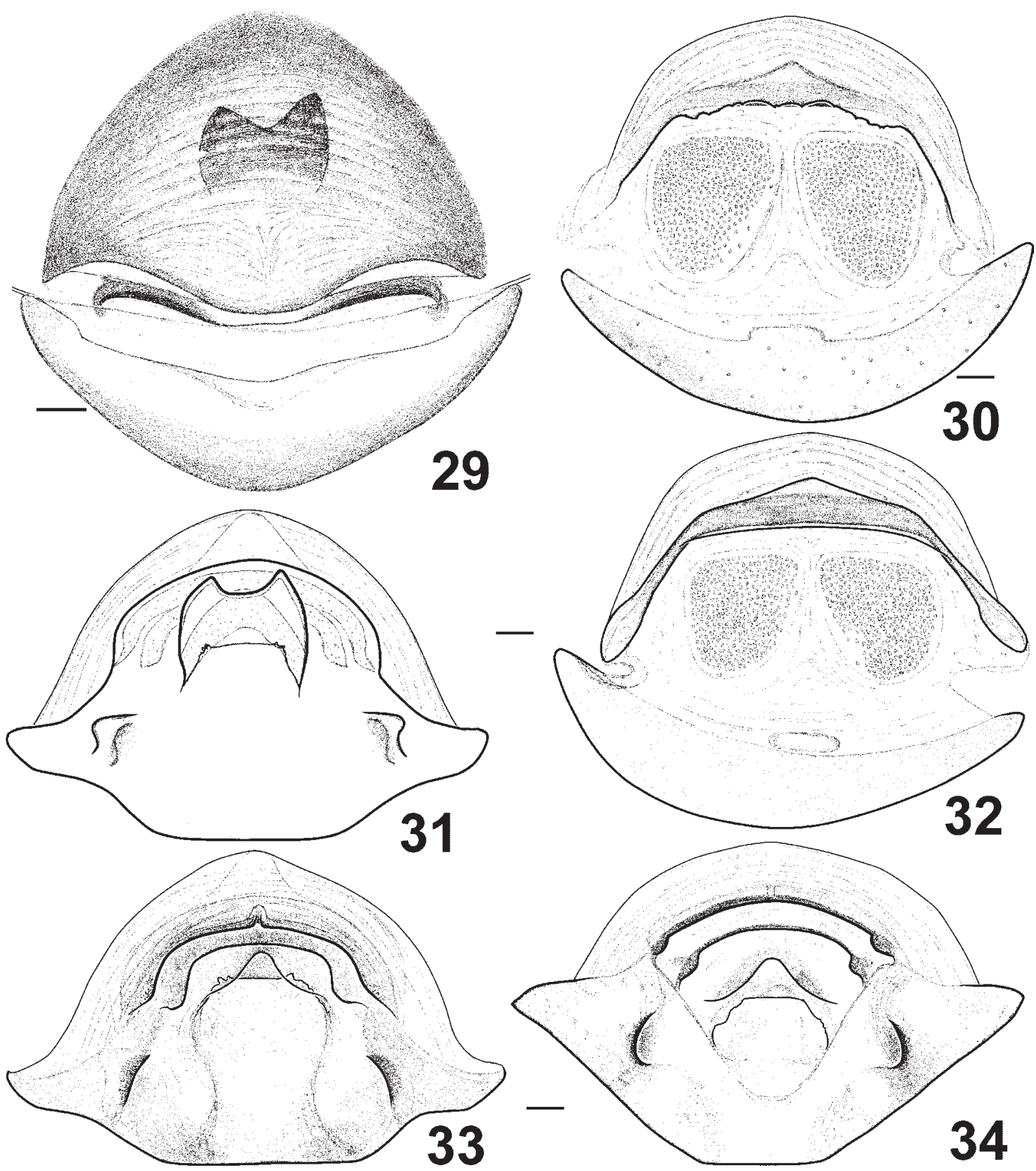

Figs 29-34. Epigyne of Hoplopholcus longipes: 29 - ventral view; 30 - dorsal part of epigyne, ventral view; 31 - ventral part of epigyne, ventral view; 32 - dorsal part of epigyne, dorsal view; 33 - ventral part of epigyne, dorsal view; 34 - ventral part of epigyne, caudal view.

Рис. 29-34. Эпигина Hoplopholcus longipes: 29 — вентрально; 30 — дорсальная часть эпигины, вентрально; 31 — вентральная часть эпигины, вентрально; 32 - дорсальная часть эпигины, дорсально; 33 - вентральная часть эпигины, дорсально; 34 вентральная часть эпигины, сзади.

1902 - sub Hyctia; Charitonov, 1932 and Mcheidze, 1997 — sub Mithion; Otto \& Dietzold, 2006].

DISTRIBUTION. Trans-Palaearctic nemoral-subtropical range [Mikhailov, 1997; Logunov \& Marusik, 2000; Helsdingen, 2010; Platnick, 2010].
Menemerus semilimbatus (Hahn, 1829)

RECORDS. Gudauta; Sukhum [Mcheidze, 1997; Otto \& Dietzold, 2006]. 
DISTRIBUTION. West Palaearctic nemoral range and Argentina [Mikhailov, 1997; Helsdingen, 2010; Platnick, 2010].

Menemerus taeniatus (L. Koch, 1867)

RECORDS. Sukhum [Spassky, 1937 and Mcheidze, 1997 — sub M. parietinus Spasski, 1934; Prószyñski, 1979; Otto \& Dietzold, 2006].

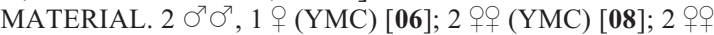
(CP) [52].

DISTRIBUTION. Mediterranean to Caucasus; Argentina [Mikhailov, 1997; Logunov \& Marusik, 2000; Helsdingen, 2010; Platnick, 2010]

Myrmarachne formicaria (De Geer, 1778)

RECORDS. Sukhum Distr. [Spassky, 1937; Mcheidze, 1997; Logunov \& Guseinov, 2002; Otto \& Dietzold, 2006].

MATERIAL. 3 ㅇ (YMC) [10]; $10^{\top}, 1$ ㅇ (YMC) [12]; 3 우

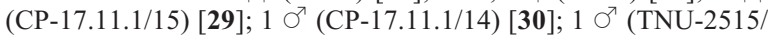
4) $[\mathbf{3 1}] ; 1$ O $^{7}$ (TNU-2639/17) [39]; 1 + (TNU-2653/48) [56].

DISTRIBUTION. Trans-Palaearctic disjuctive nemoral range (absent between Caucasus and Far East), USA (introduced) [Mikhailov, 1997; Logunov \& Marusik, 2000; Helsdingen, 2010; Platnick, 2010].

Neon levis (Simon, 1871)

RECORDS. Abkhazia unspecified [Logunov \& Guseinov, 2002; Otto \& Dietzold, 2006].

MATERIAL. $10^{7}, 3$ 우 (TNU-2639/26) [39]; 5 우 (TNU2651/40) [55]; 2 우우 (TNU-2653/51) [56].

DISTRIBUTION. West-Palaearctic nemoral range [Mikhailov, 1997; Logunov \& Marusik, 2000; Helsdingen, 2010; Platnick, 2010].

Neon reticulatus (Blackwall, 1853)

MATERIAL. 1 (TNU-2515/1) [31]; $10^{7}$ (TNU-2513/1) [32] $3 \mathrm{O}^{7} \mathrm{O}^{7}, 6$ 오 (TNU-2639/27) [39]; 5 우 (TNU-2644/22) [45]; 2 ㅇㅇ (TNU-2651/41) [55].

DISTRIBUTION. Trans-Palaearctic - West Nearctic nemoral range [Mikhailov, 1997; Logunov \& Marusik, 2000; Marusik et al., 2000; Helsdingen, 2010; Platnick, 2010].

\section{Phintella castriesiana (Grube, 1861)}

MATERIAL. 1 ( $(\mathrm{YMC})[\mathbf{1 4}] ; 1 \sigma^{7}$ (YMC) [19]; $1 \sigma^{7}(\mathrm{YMC})$ [21]; 1 О $^{7}$ (TNU-2636/58) [36]; 1 ○ (TNU-2639/18) [39]; 1 ○ (TNU-2644/18) [45]; 3 우 (TNU-2651/38) [55]; 1 O (TNU-2653/ 46) $[56]$.

DISTRIBUTION. Palaearctic nemoral disjunctive range, it is absent between Caucasus and Far East [Mikhailov, 1997; Logunov \& Marusik, 2000; Helsdingen, 2010; Platnick, 2010].

Phlegra fasciata (Hahn, 1826)

RECORDS. Sukhum [Mcheidze, 1964, 1997; Otto \& Dietzold, 2006].
MATERIAL. 2 우 (TNU-2652/6) [54].

DISTRIBUTION. Trans-Palaearctic nemoral range [Mikhailov, 1997; Logunov \& Marusik, 2000; Helsdingen, 2010; Platnick, 2010].

\section{Pseudeuophrys erratica (Walckenaer, 1826)}

RECORDS. Gagra Distr.: SW slope of Mandizyzkho [=Mamdzyshkha] Mt., $1400 \mathrm{~m}$ a.s.l. [Logunov \& Guseinov, 2002; Otto \& Dietzold, 2006].

MATERIAL. 2 우 (YMC) [10]; $2 \bigcirc^{7} \sigma^{7}, 1$ ( 1 (TNU-2636/57) [36]; $2 \bigcirc^{7} \sigma^{7}, 3$ 우 (TNU-2639/24) [39]; $1 \sigma^{7}$ (TNU-2640/8) [40]; 1 ; (TNU-2644/24) [45]; 1 우 (TNU-2645/2) [46]; 1 우 (TNU-2646/1) [47]; $10^{\top}$ (CP) [52]; 2 O $^{7}$ O $^{7}, 7$ 우 (TNU-2651/43) [55]; 2 ㅇ (CP) [62]

DISTRIBUTION. Trans-Palaearctic nemoral range [Mikhailov, 1997; Logunov \& Marusik, 2000; Helsdingen, 2010; Platnick, 2010].

Pseudicius encarpatus (Walckenaer, 1802)

MATERIAL. $1 O^{\top}$ (TNU-2651/42) [55].

DISTRIBUTION. West and Central Palaearctic nemoral range [Mikhailov, 1997; Logunov \& Marusik, 2000; Helsdingen, 2010; Platnick, 2010].

\section{Salticus mutabilis Lucas, 1846}

RECORDS. Kelasur, Sukhum [Mcheidze, 1964, 1997; Otto \& Dietzold, 2006].

MATERIAL. 1 ( (YMC) [06]; 1 ( $(\mathrm{CP})[52]$.

DISTRIBUTION. Mediterranean and Caucasus; Argentina [Mikhailov, 1997; Helsdingen, 2010; Platnick, 2010].

Sitticus relictarius Logunov, 1998

MATERIAL. 1 9 (TNU-2644/25) [45].

DISTRIBUTION. Caucasus and Iran [Mikhailov, 1997; Platnick, 2010].

Talavera aequipes (O. Pickard-Cambridge, 1871)

MATERIAL. $8 \sigma^{7} \sigma^{7}, 37$ 우 (TNU-2641/21) [41]; $2 \circlearrowleft^{\top} \sigma^{7}, 2$ 우 (TNU-2652/7) [54]

DISTRIBUTION. Palaearctic boreo-nemoral range, east to Lena River [Mikhailov, 1997; Helsdingen, 2010; Platnick, 2010].

Talavera aperta (Miller, 1971)

MATERIAL. $1 O^{\top}$ (TNU-2636/59) [36].

DISTRIBUTION. Palaearctic nemoral range, from France east to Transbaikalia [Mikhailov, 1997; Logunov \& Marusik, 2000; Helsdingen, 2010; Platnick, 2010].

SCYTODIDAE (1 genus and 1 species)

Scytodes thoracica (Latreille, 1802)

RECORDS. Sukhum, Kelasur [Spassky, 1937; Mcheidze, 1997; Otto \& Dietzold, 2006]. 
।
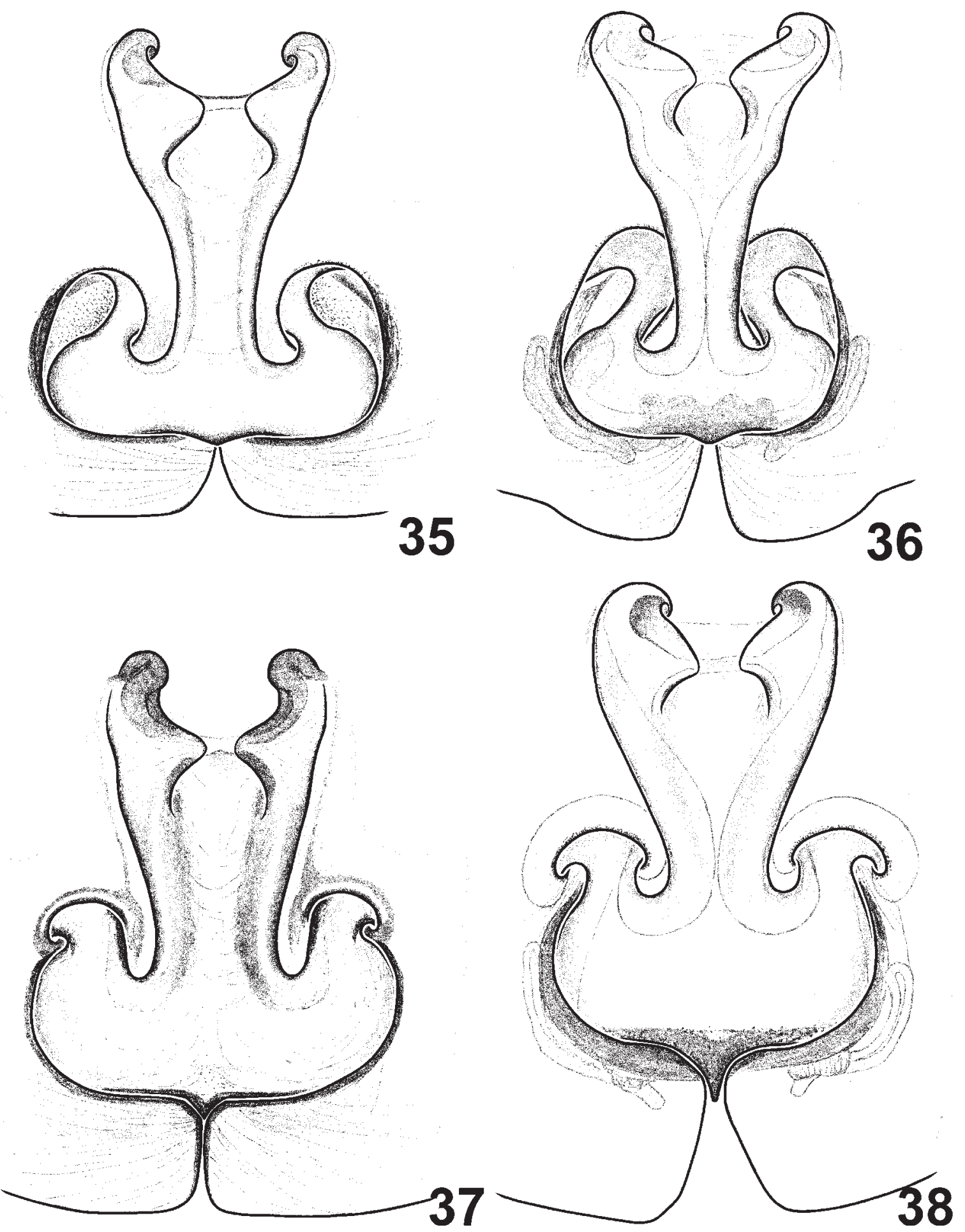

Figs 35-38. Epigynes of Pisaura species, ventral view: 35-36 - P. mirabilis from Kyiv Area of Ukraine; 37-38 — ? P. novicia from Abkhazia.

Рис. 35-38. Эпигины двух видов Pisaura, вентрально: 35-36 - P. mirabilis из Киевской области Украины; 37-38 — ? P. novicia из Абхазии. 
MATERIAL. $1 \bigcirc^{7}, 2$ ㅇ (YMC) [08]; 2 ㅇ (YMC) [24]; 1 ㅇ (TNU-2636/28) [36]; 1 q (TNU-2647/4) [48]; 1 (CP) [52]; 1 ㅇ (TNU-2651/3) [55].

DISTRIBUTION. Holarctic nemoral range [Mikhailov, 1997; Helsdingen, 2010; Platnick, 2010].

SEGESTRIIDAE (1 genus and 3 species)

Segestria bavarica C.L. Koch, 1843

RECORDS. Gudauta, Sukhum, Ochamchyra, Kodor [Spassky, 1937; Dunin, 1986; Mcheidze, 1997; Otto \& Dietzold, 2006].

DISTRIBUTION. West Palaearctic nemoral range: from Portugal east to Caucasus, and from Sweden south to Sicily and Crete [Mikhailov, 1997; Helsdingen, 2010; Platnick, 2010].

Segestria florentina (Rossi, 1790)

RECORDS. Akhali Atoni, Gagra, Sukhum, Kelasur, Ochamchyra [Spasski, 1937; Dunin, 1986 Mcheidze, 1964, 1997; Otto \& Dietzold, 2006].

MATERIAL. 1 \% (YMC) [06]; 30 o'o (YMC) [08]; 3 우 (YMC) [10]; 3 오 (CP) [59].

DISTRIBUTION. West Palaearctic nemoral range: from Portugal east to Caucasus, and from Britain south to Sicily and Crete [Mikhailov, 1997; Helsdingen, 2010; Platnick, 2010].

Segestria senoculata (Linnaeus, 1758)

RECORDS. Gagra Distr.: Chamkhona Cave near of Zhove-Kvara River [Lyovushkin, 1966].

MATERIAL. 2 우 (TNU-2636/29) [36]; $10^{7}$ (TNU-2639/10) [39]; 4 sad. . $($ TNU-2652/3) [54]; 1 (TNU-2653/20) [56].

DISTRIBUTION. West Palaearctic nemoral range [Mikhailov, 1997; Helsdingen, 2010; Platnick, 2010].

SPARASSIDAE (1 genus and 1 species)

Micrommata virescens (Clerck, 1757)

RECORDS. All sea coast [Wagner, 1896 - sub Drassus virescens]; Gulripsh Distr., Tsebelda [Simon 1899, Verzhbitsky, 1902; Mcheidze, 1997; Otto \& Dietzold, 2006]; Sukhum [Spassky, 1937].

MATERIAL. $10^{7}$ (TNU-2636/33) [36].

DISTRIBUTION. Trans-Palaearctic nemoral range [Mikhailov, 1997; Helsdingen, 2010; Platnick, 2010].

TETRAGNATHIDAE (4 genera and 8 species)

Meta bourneti Simon, 1922

RECORDS. Sukhum Distr.: Adhzaba Cave, Eshera; Gulripsh Distr.: Kelasur [Charitonov, 1936, 1939, 1941, 1947; Dzhanashvili, 1965; Mcheidze, 1964, 1997; Otto \& Dietzold, 2006].

DISTRIBUTION. West Palaearctic nemoral range: from Portugal east to Caucasus, and from Britain south to Sicily and Crete [Mikhailov, 1997; Helsdingen, 2010; Platnick, 2010].

\section{Metellina mengei (Blackwall, 1869)}

MATERIAL. $1 \bigcirc^{7}$ (TNU-2640/16) [40]; 2 ㅇ (TNU-2641/4) [41]; $10^{7}, 1$ \& (TNU-2643/4) [44].

DISTRIBUTION. West and Central Palaearctic nemoral range: from Portugal east to Ob River in West Siberia [Mikhailov, 1997; Helsdingen, 2010; Platnick, 2010].

\section{Metellina merianae (Scopoli, 1763)}

RECORDS. Sukhum Distr.: Adzaba Cave [Charitonov, 1936, 1939, 1941 — sub Meta]; Cave Adzhaba, Cave Kelasur [Charitonov, 1947]; Gudauta Distr.: Tarkily; Sukhum Distr.: Eshera [Dzhanashvili, 1965 and Mcheidze, 1964, 1997 — sub Meta; Otto \& Dietzold, 2006].

MATERIAL. 1 ( (YMC) [21]; 10 우 (YMC) [23]; $20^{7} 0^{7}, 22$ ㅇ (TNU-2636/6) [36]; $1 \bigcirc^{7}, 5$ 오 (TNU-2639/5) [39]; 1 ㅇ (CP) [53]; $8 \bigcirc^{7} \bigcirc^{\top}, 27$ 우 (TNU-2651/54) [55]; 1 우 (TNU-2653/19) [56]; 5 우 (CP) $[\mathbf{6 2}]$.

DISTRIBUTION. European nemoral range: from Portugal east to Ural [Mikhailov, 1997; Helsdingen, 2010; Platnick, 2010].

Metellina segmentata (Clerck, 1757)

RECORDS. Sukhum [Mcheidze, 1997 — sub Meta; Otto \& Dietzold, 2006].

MATERIAL. 2 우 (ZMMU) [33]; 1 오 (TNU-2639/6) [39]; 1 우 (TNU-2644/12) [45]; $1 \mathrm{O}^{7}(\mathrm{CP})[\mathbf{6 0}]$.

DISTRIBUTION. West-Palaearctic nemoral range, and Canada (introduced) [Mikhailov, 1997; Helsdingen, 2010; Platnick, 2010].

Pachygnatha degeeri Sundevall, 1830

RECORDS. Eshera, Sukhum [Spassky, 1937; Mcheidze, 1997; Otto \& Dietzold, 2006].

MATERIAL. $100 \mathrm{O}^{7} \mathrm{O}^{7}, 117$ 우 (TNU-2653/33) [56].

DISTRIBUTION. Trans-Palaearctic nemoral range [Mikhailov, 1997; Helsdingen, 2010; Platnick, 2010].

\section{Tetragnatha montana Simon, 1874}

RECORDS. Akhali Atoni, Sukhum [Mcheidze, 1997; Otto \& Dietzold, 2006].

MATERIAL. 1 \% (CP-38.12.1/10) [03].

DISTRIBUTION. Trans-Palaearctic nemoral range [Mikhailov, 1997; Helsdingen, 2010; Platnick, 2010].

Tetragnatha nigrita Lendl, 1886

RECORDS. Gudauta; Gulripsh; Sukhum [Spassky, 1937; Mcheidze, 1997; Otto \& Dietzold, 2006].

DISTRIBUTION. Trans-Palaearctic nemoral range [Mikhailov, 1997; Helsdingen, 2010; Platnick, 2010]. 
।
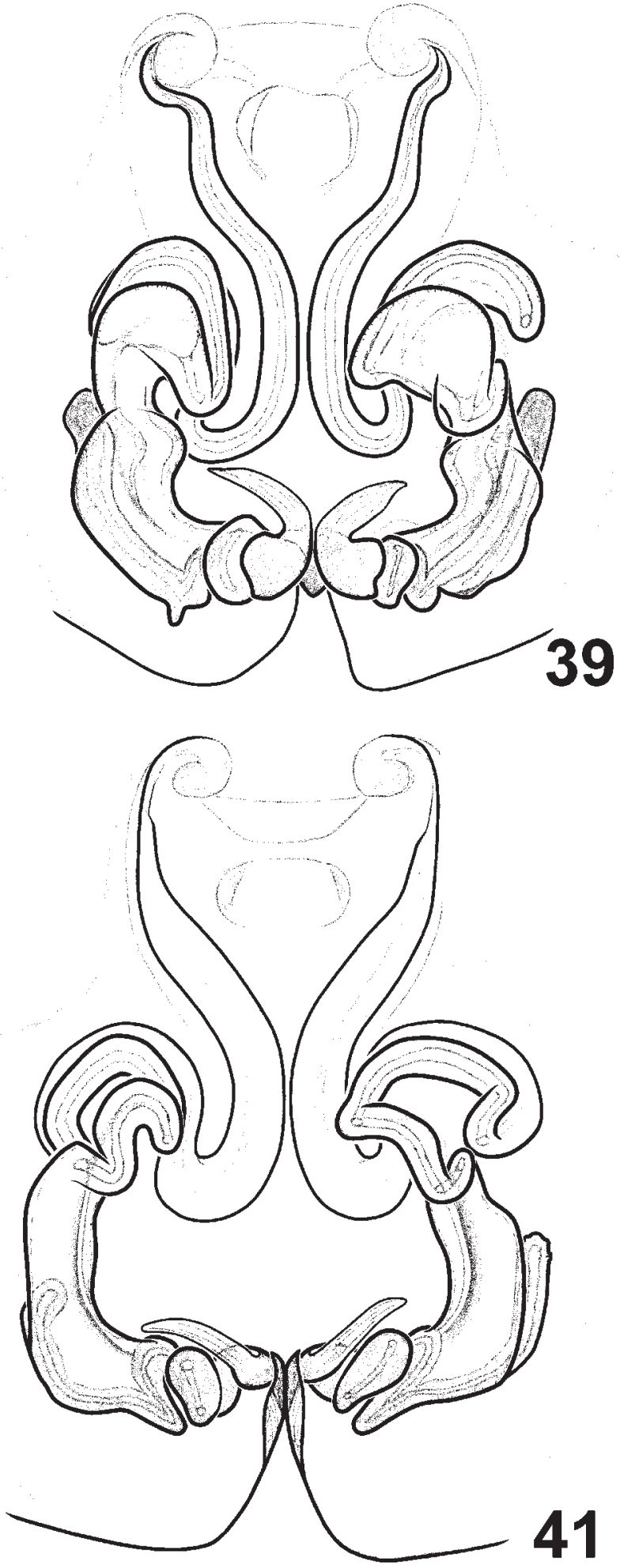
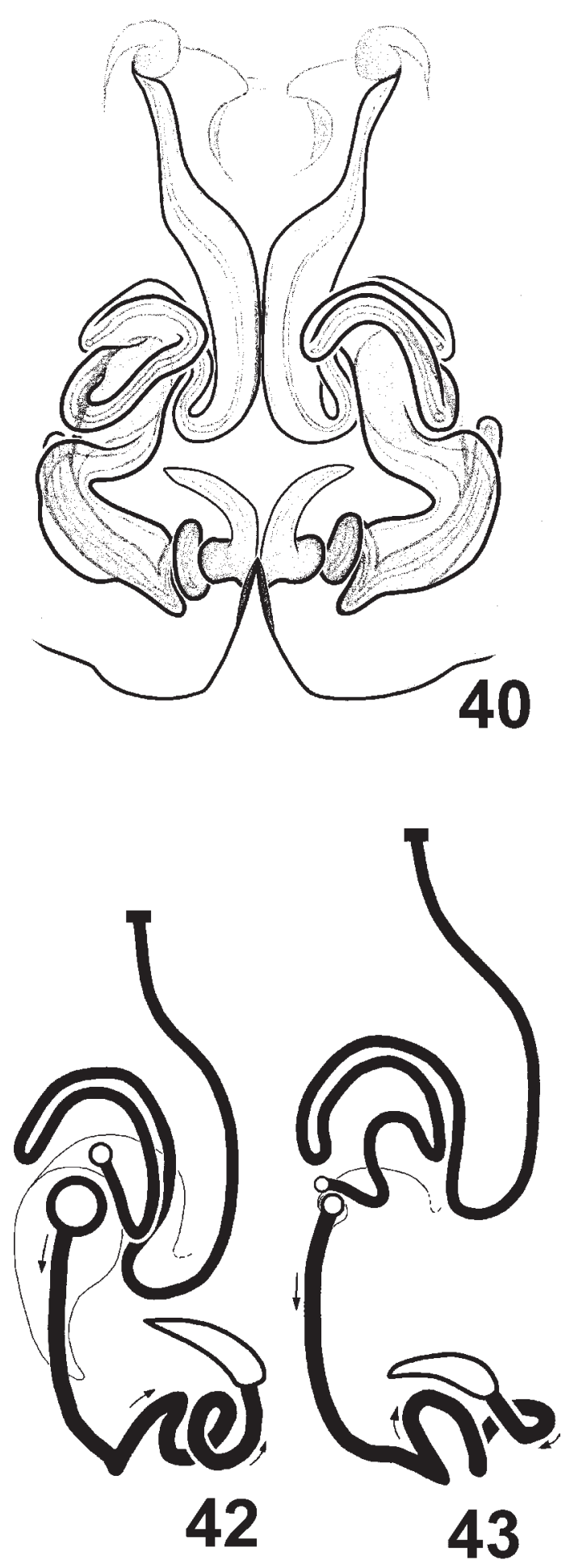

Figs. 39-43. Epigynes of Pisaura species, dorsal view: 39-40 - P. mirabilis from Kyiv Area of Ukraine; $41-$ ? P. novicia from Abkhazia; 42 - schematic course of spermathecae duct traectory of $P$. mirabilis from Kyiv Area of Ukraine; 43 - schematic course of spermathecae duct traectory of ? P. novicia from Abkhazia.

Рис. 39-43. Эпигины двух видов Pisaura, дорсально: 39-40-P. mirabilis из Киевской области Украины; 41 - ? P. novicia из Абхазии; 42 - схема траектории каналов сперматеки P. mirabilis из Киевской области Украины; 43 - схема траектории каналов сперматеки ? P. novicia из Абхазии. 
Tetragnatha obtusa C.L. Koch, 1837

RECORDS. Sukhum, Kelasur, [Mcheidze, 1964, 1997; Otto \& Dietzold, 2006].

DISTRIBUTION. Trans-Palaearctic nemoral range [Mikhailov, 1997; Helsdingen, 2010; Platnick, 2010].

THERIDIIDAE (20 genera and 31 species)

Anelosimus pulchellus (Walckenaer, 1802)

RECORDS. Akhali Atoni, Sukhum [Mcheidze, 1997; Otto \& Dietzold, 2006].

DISTRIBUTION. West Palaearctic nemoral range [Mikhailov, 1997; Helsdingen, 2010; Platnick, 2010].

Asagena phalerata (Panzer, 1801)

MATERIAL. $5 \bigcirc^{7} O^{7}, 27$ 오 (TNU-2652/22) [54]; 1 오 (CP) [62]; 1 $(\mathrm{CP})[63]$.

DISTRIBUTION. Trans-Palaearctic nemoral range [Mikhailov, 1997; Helsdingen, 2010; Platnick, 2010].

Crustulina guttata (Wider, 1834)

MATERIAL. $8 \sigma^{7} \sigma^{7}, 10$ 우 (YMC) [07]; $11 \bigcirc^{7}+$ (YMC) [10]; 1 (YMC) [11]; 55 O $^{7}+$ (YMC) [12]; $1 \bigcirc^{7}, 3$ 우 (TNU-2653/55) [56].

DISTRIBUTION. Trans-Palaearctic nemoral range [Mikhailov, 1997; Helsdingen, 2010; Platnick, 2010].

Cryptachaea riparia (Blackwall, 1834)

MATERIAL. 10 우 (TNU-2653/64) [56].

DISTRIBUTION. Trans-Palaearctic nemoral range [Mikhailov, 1997; Helsdingen, 2010; Platnick, 2010].

Dipoena melanogaster (C.L. Koch, 1837)

MATERIAL. $2 \bigcirc^{7} \sigma^{\top}, 1$ ㅇ (TNU-2639/47) [39]; 2 우 (TNU2653/53) [56].

DISTRIBUTION. West Palaearctic nemoral range [Mikhailov, 1997; Helsdingen, 2010; Platnick, 2010].

Enoplognatha ovata (Clerck, 1757)

RECORDS. Sukhum [Mcheidze, 1997 — sub Theridium; Otto \& Dietzold, 2006].

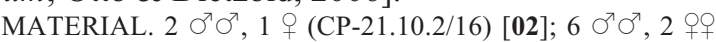
(TNU-2636/62) [36]; 5 O $^{7} \sigma^{7}$ (TNU-2639/43) [39]; $1 \sigma^{7}$ (TNU2640/14) [40]; 2 ○ O $^{7}, 6$ 우우 (TNU-2644/32) [45]; 2 ○ $^{7} \sigma^{7}, 4$ 우우 (TNU-2651/16) [55]; 4 O $^{\top} \sigma^{\top}, 13$ 우 (TNU-2653/56) [56].

DISTRIBUTION. West Palaearctic and Nearctic nemoral range [Mikhailov, 1997; Helsdingen, 2010; Platnick, 2010].

Enoplognatha quadripunctata Simon, 1884

MATERIAL. 1 (CP) [62].

DISTRIBUTION. Mediterranean-Caucasian range: from Portugal east to Azerbaijan [Helsdingen, 2010; Platnick, 2010].
Episinus maculipes Cavanna, 1876

MATERIAL. $2 O^{\top} O^{\top}$ (TNU-2651/17) [55]; 3 우 (TNU-2653/ 57) $[56]$.

DISTRIBUTION. Mediterranean-Crimean-Caucasian range [Helsdingen, 2010; Platnick, 2010].

Episinus truncatus Latreille, 1809

RECORDS. Sukhum Distr.: Adzaba Cave, Eshera; Gulripsh Distr.: Kelasur [Charitonov, 1936, 1939, 1941, 1947; Spassky, 1937; Dzhanashvili, 1965; Mcheidze, 1997; Otto \& Dietzold, 2006].

MATERIAL. 2 우 (YMC) [22]; 1 ○ा (TNU-2514/2) [32]; 2 $O^{7} O^{7}, 2$ 우 (TNU-2636/64) [36]; $2 O^{7} O^{7}$ (TNU-2644/33) [45]; 14 $\sigma^{7} \sigma^{7}, 10$ 우 (TNU-2651/18) [55]; 6 ○ $^{7} \sigma^{7}, 8$ 우 (TNU-2653/58) [56].

DISTRIBUTION. West Palaearctic nemoral range [Mikhailov, 1997; Helsdingen, 2010; Platnick, 2010].

Euryopis flavomaculata (C.L. Koch, 1836)

MATERIAL. 1 (TNU-2514/5) [32].

DISTRIBUTION. Trans-Palaearctic nemoral range [Mikhailov, 1997; Helsdingen, 2010; Platnick, 2010].

Neottiura bimaculata (Linne, 1767)

MATERIAL. 2 우 (TNU-2636/68) [36]; 5 ㅇ (TNU-2652/24) [54]; 1 ㅇ (TNU-2651/20) [55].

DISTRIBUTION. Circum-Holarctic nemoral range [Mikhailov, 1997; Marusik et al., 2000; Helsdingen, 2010; Platnick, 2010].

Paidiscura pallens (Blackwall, 1834)

MATERIAL. 1 \% (TNU-2639/50) [39].

DISTRIBUTION. West Palaearctic nemoral range [Mikhailov, 1997; Helsdingen, 2010; Platnick, 2010].

Parasteatoda lunata (Clerck, 1757)

RECORDS. Sukhum [Mcheidze, 1964, Otto \& Dietzold, 2006]. [55].

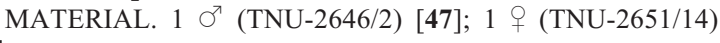

DISTRIBUTION. Trans-Palaearctic nemoral range [Mikhailov, 1997; Helsdingen, 2010; Platnick, 2010].

Parasteatoda simulans (Thorell, 1875)

MATERIAL. 4 우 (CP-21.10.27/9) [51]; 1 + (CP) [53]; 4 $O^{\top} O^{\top}, 17$ 우우 (TNU-2653/65) [56].

DISTRIBUTION. Trans-Palaearctic nemoral range [Mikhailov, 1997; Helsdingen, 2010; Platnick, 2010].

Parasteatoda tabulata (Levi, 1980)

MATERIAL. $1 \sigma^{7}, 1+$ (TNU-2647/1) [48].

DISTRIBUTION. Holarctic nemoral range [Mikhailov, 1997; Helsdingen, 2010; Platnick, 2010]. 
Parasteatoda tepidariorum (C.L. Koch, 1841)

RECORDS. Akhali Atoni, Sukhum [Spassky, 1937 — sub Theridium; Mcheidze, 1964, 1997 and Otto \& Dietzold, 2006 — sub Achaearanea].

MATERIAL. 1 ( $(\mathrm{YMC})[\mathbf{1 8}] ; 17 \mathrm{O}^{7} \mathrm{O}^{7}, 15$ 우 (TNU-2636/60) [36]; 2 우 (TNU-2638/3) [38]; 6 O $^{7} \bigcirc^{7}, 5$ 우 (TNU-2639/45) [39]; 1 ○', 2 우오 (TNU-2640/13) [40]; 1 ○', 5 우우 (TNU-2644/34) [45]; 11 O $^{7}, 11$ 우 (TNU-2651/15) [55]; 1 O$^{7}, 7$ 우 (CP) [62].

DISTRIBUTION. Cosmopolitan [Mikhailov, 1997; Helsdingen, 2010; Platnick, 2010].

Phoroncidia minuta (Spassky, 1932)

RECORDS. Gagra, Kelasur [Mcheidze, 1964, 1997 - sub Ulesania; Otto \& Dietzold, 2006].

MATERIAL. 2 +9 (TNU-2651/19) [55].

DISTRIBUTION. Caucasus: from Krasnodar Area (Khosta) east to Azerbaijan [Spassky, 1932, 1937; Logunov \& Marusik, 1992; Mikhailov, 1997; Helsdingen, 2010; Platnick, 2010].

\section{Phylloneta impressa (C.L. Koch, 1881)}

RECORDS. Sukhum [Spassky, 1937 and Mcheidze, 1997 — sub Theridium; Otto \& Dietzold, 2006].

MATERIAL. $20^{7} \sigma^{7}, 4$ o (TNU-2652/25) [54].

DISTRIBUTION. Holarctic polyzonal range [Mikhailov, 1997; Helsdingen, 2010; Platnick, 2010].

Platnickia tincta (Walckenaer, 1802)

RECORDS. Sukhum [Spassky, 1937 - sub Theridium; Mcheidze, 1997 — sub Theridium; Otto \& Dietzold, 2006 - sub Keijia].

MATERIAL. 1 (TNU-2639/51) [39]; 1 ㅇ (TNU-2651/21) [55]; $2 \bigcirc^{7} 0^{7}, 3$ 우 (TNU-2653/60) [56].

DISTRIBUTION. West Palaearctic - Nearctic nemoral range [Mikhailov, 1997; Helsdingen, 2010; Platnick, 2010].

Robertus mediterraneus Eskov, 1987

MATERIAL. $4 \sigma^{7} \sigma^{7}, 1$ (TNU-2639/49) [39].

DISTRIBUTION. Mediterranean-Caucasian range: from France east to Caucasus [Mikhailov, 1997; Helsdingen, 2010; Platnick, 2010].

Rhomphaea hyrcana (Logunov \& Marusik, 1990)

MATERIAL. $1 O^{7}$ (TNU-2653/54) [56]

DISTRIBUTION. Caucasus-Far East disjunctive range [Logunov \& Marusik, 1990; Mikhailov, 1997; Helsdingen, 2010; Platnick, 2010].

Steatoda albomaculata (De Geer, 1778)

RECORDS. Eshera, Sukhum, Kelasur [Mcheidze, 1964, 1997 — sub Lithyphantes; Otto \& Dietzold, 2006].
DISTRIBUTION. Circum-Holarctic polyzonal range [Mikhailov, 1997; Helsdingen, 2010; Platnick, 2010].

\section{Steatoda bipunctata (Linnaeus, 1758)}

MATERIAL. $20^{7} \bigcirc^{7}, 5$ ㅇ (TNU-2652/23) [54].

DISTRIBUTION. Holarctic (East Nearctic-TransPalaearctic) nemoral range [Mikhailov, 1997; Marusik et al., 2000; Helsdingen, 2010; Platnick, 2010].

\section{Steatoda paykulliana (Walckenaer, 1805)}

RECORDS. Gagra, Sukhum [Simon, 1899; Verzhbitsky, 1902 and Mcheidze, 1964, 1997 — all sub Lithyphantes; Otto \& Dietzold, 2006].

MATERIAL. 2 ㅇ (CP-21.13.3/8) [03]; $1 O^{7}$ (YMC) [06]; 1 우 (CP-21.13.3/12) [09]; $1 \sigma^{7}$ (YMC) [20]; 3 우 (TNU-2636/41) [36]; $1 O^{7}(\mathrm{CP})[\mathbf{5 2}] ; 1 O^{7}(\mathrm{CP})$ [53].

DISTRIBUTION. West and Central Palaearctic nemoral range [Mikhailov, 1997; Helsdingen, 2010; Platnick, 2010].

Steatoda triangulosa (Walckenaer, 1802)

RECORDS. Sukhum [Spassky, 1937; Mcheidze, 1964, 1997; Otto \& Dietzold, 2006].

MATERIAL. 1 ㅇ (YMC) [06]; 2 우 (TNU-2638/4) [38]; 4 우 (TNU-2647/2) [48]; 2 우 (CP-21.14.2/10) [50]; 2 우 (CP-21.14.2/ 9) [51]; 1 아 (CP) [52]; 5 우 (CP) [53]; $3 \mathrm{O}^{7} \bigcirc^{7}, 3$ 우 (CP) [59].

DISTRIBUTION. Cosmopolitan [Mikhailov, 1997; Helsdingen, 2010; Platnick, 2010].

Theridion cinereum Thorell, 1875

RECORDS. Gudauta [Mcheidze, 1946, 1997 — sub Theridium; Otto \& Dietzold, 2006].

DISTRIBUTION. East Mediterranean and Caucasus: from the Balkans east to Azerbaijan, north to Austria and south to Turkey [Marusik et al., 2009].

Theridion pinastri L. Koch, 1872

MATERIAL. $10^{\top}, 1$, 1 (TNU-2639/48) [39]; $10^{\top}, 2$ ㅇ (TNU2653/61) [56].

DISTRIBUTION. Trans-Palaearctic nemoral range [Mikhailov, 1997; Helsdingen, 2010; Platnick, 2010].

Theridion varians Hahn, 1833

MATERIAL. $1 \sigma^{7}$ (TNU-2638/5) [38]; $1 \sigma^{7}, 6$ ㅇ (TNU-2639/ 42) [39]; 1 O $^{7}$ (TNU-2652/26) [54]; $1 \mathrm{O}^{7}, 1$ ㅇ (TNU-2651/22) [55]; 6 우 (TNU-2653/59) [56].

DISTRIBUTION. Trans-Palaearctic - West-Nearctic boreo-nemoral range [Mikhailov, 1997; Marusik et al., 2000; Helsdingen, 2010; Platnick, 2010].

Theridula gonygaster (Simon, 1873)

RECORDS. Sukhum [Marusik, 1989]. MATERIAL. $2 \sigma^{7} \sigma^{7}$ (TNU-2653/81) [56]. 
DISTRIBUTION. Cosmopolitan [Mikhailov, 1997; Helsdingen, 2010; Platnick, 2010].

Theridula opulenta (Walckenaer, 1842)

RECORDS. Gagra, Sukhum [Mcheidze, 1964, 1997; Otto \& Dietzold, 2006].

DISTRIBUTION. Cosmopolitan [Mikhailov, 1997; Helsdingen, 2010; Platnick, 2010].

Yaginumena maculosa (Yoshida et Ono, 2000)

RECORDS. Pitsunda [Marusik et al., 2004; Otto \& Dietzold, 2006].

DISTRIBUTION. Caucasus and Japan disjunctive range [Mikhailov, 1997; Helsdingen, 2010; Platnick, 2010].

THERIDIOSOMATIDAE (1 genus and 1 species)

Theridiosoma gemmosum (L. Koch, 1877)

MATERIAL. 6 우 (TNU-2651/23) [55].

DISTRIBUTION. East Nearctic and West Palaearctic nemoral range [Mikhailov, 1997; Helsdingen, 2010; Platnick, 2010].

THOMISIDAE (10 genera and 20 species)

Cozyptila guseinovorum Marusik et Kovblyuk, 2005

RECORDS. Gagra Distr.: Gegha River [Kovblyuk \& Ponomarev, 2008].

MATERIAL. 1 ( $($ YMC) $[24] ; 19$ (TNU-2514/1) [32]; 6 우 (TNU-2636/32) [36]; 1 O' (TNU-2639/40) [39]; 3 아 (TNU-2644/ 5) $[\mathbf{4 5}] ; 1$ q (TNU-2651/5) [55]; 8 우 (TNU-2653/6) [56].

DISTRIBUTION. Turkey, Crimea and Caucasus [Helsdingen, 2010; Platnick, 2010].

Ebrechtella tricuspidata (Fabricius, 1775)

RECORDS. Gagra; Sukhum [Spassky, 1937 - sub Misumena; Mcheidze, 1997 and Otto \& Dietzold, 2006 — sub Misumenops].

MATERIAL. $1 \sigma^{7}$ (TNU-2639/38) [39]; $1 \sigma^{7}$ (CP-19.18.1/12) [49]; 1 \& (TNU-2653/16) [56].

DISTRIBUTION. Trans-Palaearctic nemoral range [Mikhailov, 1997; Helsdingen, 2010; Platnick, 2010].

Misumena vatia (Clerck, 1757)

RECORDS. Sukhum [Spassky, 1937; Mcheidze, 1997; Otto \& Dietzold, 2006].

MATERIAL. $1 \sigma^{7}$ (CP-19.17.1/14) [02]; 2 O $^{7} \sigma^{7}$ (TNU-2639/ 39) [39]; $1 \mathrm{O}^{7}$ (TNU-2640/10) [40]; $1 \mathrm{O}^{7}$ (TNU-2643/1) [44]; 2 $\mathrm{O}^{7} \bigcirc^{7}, 1$ ( 1 (TNU-2644/7) [45]; 2 우 (TNU-2652/8) [54]; $2 \mathrm{O}^{7} \mathrm{O}^{7}, 1$, 1 (T) (TNU-2653/13) [56]

DISTRIBUTION. Circum-Holarctic polyzonal range [Mikhailov, 1997; Marusik et al., 2000; Helsdingen, 2010; Platnick, 2010].
Ozyptila atomaria (Panzer, 1801)

MATERIAL. $20^{7} 0^{7}, 2$ 우 (YMC) [27].

DISTRIBUTION. Trans-Palaearctic boreo-nemoral range [Mikhailov, 1997; Helsdingen, 2010; Platnick, 2010].

Ozyptila trux (Blackwall, 1846)

MATERIAL. 1 (TNU-2652/12) [54].

DISTRIBUTION. Trans-Palaearctic nemoral range and Canada (introduced) [Mikhailov, 1997; Helsdingen, 2010; Platnick, 2010].

Pistius truncatus (Pallas, 1772)

RECORDS. Sukhum [Mcheidze, 1997; Otto \& Dietzold, 2006].

DISTRIBUTION. West Palaearctic nemoral range [Mikhailov, 1997; Helsdingen, 2010; Platnick, 2010].

Runcinia grammica (C.L. Koch, 1837)

RECORDS. Sukhum [Spassky, 1937 - sub R. lateralis C.L. Koch], Eshera, Ritsa Lake [Mcheidze, 1997 — sub R. lateralis; Otto \& Dietzold, 2006].

MATERIAL. $7 \Im^{7} \sigma^{7}, 1$ (TNU-2636/34) [36]; 1 ( 1 (TNU2653/15) [56].

DISTRIBUTION. Trans-Palaearctic nemoral range, St. Helena, South Africa [Mikhailov, 1997; Helsdingen, 2010; Platnick, 2010].

Synema caucasicum Utochkin, 1960

RECORDS. Sukhum [Mcheidze, 1997; Otto \& Dietzold, 2006].

MATERIAL. 1 (TNU-2653/18) [56].

DISTRIBUTION. West Caucasus [Mikhailov, 1997; Platnick, 2010].

NOTE. Status of species is not clear.

Synema globosum (Fabricius, 1775)

RECORDS. Gulripsh Distr., Tsebelda [Verzhbitsky, 1902]; Sukhum [Spassky, 1937], Dranda, Gudauta, Gulripsh, Sukhum [Mcheidze, 1997; Otto \& Dietzold, 2006].

MATERIAL. 1 (CP-19.19.1/7) [02]; 2 ㅇ (CP-19.19.1/9) [29]; $2 \bigcirc^{7} \sigma^{7}, 2$ 우 (TNU-2636/36) [36]; $1 \bigcirc^{7}, 2$ 우 (TNU-2639/41) [39]; $1 \mathrm{O}^{\top}$ (CP) [61].

DISTRIBUTION. Trans-Palaearctic nemoral range [Mikhailov, 1997; Helsdingen, 2010; Platnick, 2010].

Thomisus onustus Walckenaer, 1805

RECORDS. Ritsa Lake, Sukhum [Mcheidze, 1997; Otto \& Dietzold, 2006].

DISTRIBUTION. West-Central Palaearctic nemoral range [Mikhailov, 1997; Helsdingen, 2010; Platnick, 2010]. 
Tmarus horvathi Kulczyñski, 1895

MATERIAL. 1 (TNU-2636/35) [36].

DISTRIBUTION. Palaearctic disjunctive nemoral range, it is unknown between Caucasus and Far East [Mikhailov, 1997; Helsdingen, 2010; Platnick, 2010].

Tmarus stellio Simon, 1875

RECORDS. Sukhum [Spassky, 1937; Mcheidze, 1997; Otto \& Dietzold, 2006].

MATERIAL. 1 (CP-19.20.2/1) [03]; 1 (CP-19.20.2/2) [05]; 1 ㅇ (TNU-2639/37) [39]; 2 ㅇ (TNU-2653/14) [56].

DISTRIBUTION. Mediterranean and Caucasus: from Spain east to Caucasus [Mikhailov, 1997; Helsdingen, 2010; Platnick, 2010].

Xysticus acerbus Thorell, 1872

RECORDS. Gulripsh Distr.: Tsebelda [Simon, 1899; Verzhbitsky, 1902; Mcheidze, 1997; Otto \& Dietzold, 2006].

DISTRIBUTION. West Palaearctic nemoral range [Mikhailov, 1997; Helsdingen, 2010; Platnick, 2010].

Xysticus cristatus (Clerck, 1757)

RECORDS. Gulripsh, Sukhum [Mcheidze, 1997; Otto \& Dietzold, 2006].

DISTRIBUTION. Trans-Palaearctic nemoral range [Mikhailov, 1997; Helsdingen, 2010; Platnick, 2010]. $1971^{*}$

Xysticus kochi abchasicus Mcheidze et Utochkin,

RECORDS. Sukhum Distr.: Kelasur [Mcheidze, Utochkin, 1971; Mcheidze, 1997; Otto \& Dietzold, 2006].

NOTE. Probably it can be considered as a separate species, but not a subspecies.

DISTRIBUTION. West Caucasus [Mikhailov, 1997; Helsdingen, 2010; Platnick, 2010].

Xysticus laetus Thorell, 1875

RECORDS. Sukhum [Logunov, 2006].

DISTRIBUTION. Wide Mediterranean: from Italy east to Caucasus and Iran [Logunov, 2006].

Xysticus lanio C.L. Koch, 1835

RECORDS. Kelasur [Mcheidze, 1997; Otto \& Dietzold, 2006]. [56].

MATERIAL. $1 \sigma^{7}$ (TNU-2636/37) [36]; $1 \bigcirc^{7}$ (TNU-2653/17)

DISTRIBUTION. Palaearctic nemoral range, it is distributed west of Lena River [Mikhailov, 1997; Helsdingen, 2010; Platnick, 2010].

Xysticus luctator L. Koch, 1870

MATERIAL. $4 O^{7} \sigma^{7}(\mathrm{CP}-19.10 .20 / 16)[05]$.
DISTRIBUTION. West and Central Palaearctic nemoral range [Mikhailov, 1997; Helsdingen, 2010; Platnick, 2010].

Xysticus spasskyi Utochkin, 1968

MATERIAL. $2 \sigma^{\top} \sigma^{\top}, 1$ (TNU-2641/8) [41]; 2 우 (TNU2652/10) [54].

DISTRIBUTION. Crimea and Caucasian range [Mikhailov, 1997; Helsdingen, 2010; Platnick, 2010].

Xysticus ukrainicus Utochkin, 1968

MATERIAL. $1 \sigma^{\top}(\mathrm{CP}-19.10 .10 / 3)[\mathbf{0 2}] ; 2 \sigma^{\top} \sigma^{7}, 1$ (TNU2641/9) [41]; 1 o' (TNU-2651/26) [55].

DISTRIBUTION. Crimea and Caucasian range [Mikhailov, 1997; Helsdingen, 2010; Platnick, 2010].

NOTE. Records from Slovakia and Russian Plain [Mikhailov, 1997; Helsdingen, 2010] are doubtful.

ULOBORIDAE (2 genera and 3 species)

Hyptiotes flavidus (Blackwall, 1862)

RECORDS. Sukhum, Kelasur [Mcheidze, 1997 and Otto \& Dietzold, 2006 - sub H. gerhardti Wiehle, 1929].

MATERIAL. 1 \% (YMC) [22]; $10^{7}$ (YMC) [28].

DISTRIBUTION. Balkans (Greece) and Caucasus [Helsdingen, 2010; Platnick, 2010].

? Hyptiotes paradoxus (C.L. Koch, 1834)

RECORDS. Sukhum [Spassky, 1937; Mcheidze, 1997; Otto \& Dietzold, 2006].

DISTRIBUTION. West and Central Palaearctic boreo-nemoral range [Mikhailov, 1997; Helsdingen, 2010; Platnick, 2010].

NOTE. Probably records of this species from Abkhazia are based on misidentifications and refer to $H$. flavidus.

Uloborus walckenaerius (Latreille, 1806)

RECORDS. Sukhum [Spassky, 1937; Mcheidze, 1964, 1997; Otto \& Dietzold, 2006].

DISTRIBUTION. Trans-Palaearctic nemoral range [Mikhailov, 1997; Helsdingen, 2010; Platnick, 2010].

ZODARIIDAE (1 genus and 1 species)

Zodarion abantense Wunderlich, 1979

RECORDS. Pitsunda, Sukhum [Marusik, 2005; Otto \& Dietzold, 2006].

MATERIAL. $1 \bigcirc$ (YMC) [06]; $35 \sigma^{7} \bigcirc$ (YMC) $[\mathbf{1 0}] ; 11 \sigma^{70} q$ (YMC) $[12] ; 4 \bigcirc^{7} \bigcirc^{7}$ (YMC) [17]; $14 \bigcirc^{7}+$ (YMC) [24]; 1 + (CP) [62].

DISTRIBUTION. Turkey and West Caucasus [Mikhailov, 1997; Platnick, 2010]. 
ZORIDAE (1 genus and 2 species)

Zora armillata Simon, 1878

MATERIAL. 1 (ZMMU) [09].

DISTRIBUTION. West and Central Palaearctic nemoral range [Mikhailov, 1997; Helsdingen, 2010; Platnick, 2010].

Zora spinimana (Sundevall, 1833)

MATERIAL. 8 우 (TNU-2653/11) [56]

DISTRIBUTION. Trans-Palaearctic nemoral range [Mikhailov, 1997; Helsdingen, 2010; Platnick, 2010].

ZOROPSIDAE (1 genus and 1 species)

Zoropsis spinimana (Dufour, 1820)

RECORDS. Sukhum, Kelasur [Marusik, Kovblyuk, 2004; Otto \& Dietzold, 2006].

MATERIAL. $2 \sigma^{7} \sigma^{7}, 6$ 우 (YMC) [06]; $1 \odot^{7}, 3$ 우 (YMC) [08]; $1 \mathrm{O}^{7}, 1$ + (YMC) [12]; 1 O $^{7}, 1$ + (YMC) [24]; 1 + (YMC) [26]; 10 (YMC) [28].

DISTRIBUTION. Mediterranean; introduced in Caucasus (Sochi and Sukhum) and USA [Marusik, Kovblyuk, 2005; Marusik, 2005; Ponomarev, 2009; Helsdingen, 2010; Platnick, 2010].

ZYGIELLIDAE (2 genera and 2 species)

Parazygiella montana (C.L. Koch, 1834)

MATERIAL. $20^{7} \sigma^{\top}, 4$ 우 (TNU-2652/18) [54].

DISTRIBUTION. West Palaearctic nemoral range [Mikhailov, 1997; Helsdingen, 2010; Platnick, 2010].

\section{Zygiella $x$-notata $($ Clerck, 1757)}

RECORDS. Sukhum [Spassky, 1937 - sub Zilla; Mcheidze, 1997; Otto \& Dietzold, 2006].

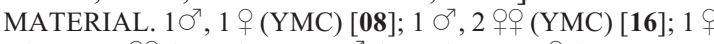
(YMC) [18]; 4 +ᄋ (YMC) [25]; $1 \mathrm{O}^{7}$ (YMC) [26]; 1 + (CP-27.12.4 1) $[\mathbf{5 1}]$.

DISTRIBUTION. West Palaearctic; amphi-Nearctic and Neotropical (introduced) [Mikhailov, 1997; Helsdingen, 2010; Platnick, 2010].

\section{Conclusions}

According to literature data and material presented here, the known spider fauna of Abkhazia includes at least 283 species (Table 1) in comparison to 153 known before 2010. Eleven additional families and 130 species have been found new to Abkhazia. In the material collected by us in Abkhazia, there are several dozens species identified to genus or even family level only. This fact clearly indicates that species diversity in Abkhazia is much higher.

Identified species can be split into 12 chorological groups (Table 2). Widely distributed species (Cosmopolitan, Holarctic, Palaearctic) compose more than $70 \%$
(203 species). Caucasian species are represented by $12 \%$ (33 species), but this figure will be higher thanks to unidentified species (see Table 1). The overage value of endemic species in Caucasus is 22\% [Marusik et al., 2006]. Also, the Ancient Mediterranean species represent an important chorological element: $8.5 \%$ (24 species). Six species have disjunctive ranges (they are unknown between Caucasus and Far East). $6 \%$ or 17 species have small-scale ranges around Caucasus (Crimea-Caucasus, Turkey-Caucasus, Crimea-Turkey-Caucasus, Turkey-Caucasus-Middle Asian Mts.-Iran, Iran-Caucasus, Crimea-Caucasus-Iran-Middle Asian Mts.).

Occurrence of additional families in Abkhazia is not very likely, although there are some possibilities to found there. For example, Selenopidae (Selenops radiatus Latreille, 1819 is widespread in Mediterranean Region including Anatolia) and Anapidae (Comaromma simoni Bertkau, 1889, is widespread in Europe, although absent in Bulgaria, or Zangrella apuliae (Caporiacco, 1949) which is known from Italy to Anatolia). Records of numerous genera and species are very likely. We expect occurrence of the following genera and species (these taxa are known in neighbouring territories): Gnaphosidae - Callilepis Westring, 1874 (1-2 species), Parasyrisca Schenkel, 1963 (1-2 species), Micaria (not fewer than 5 species); Dictynidae - more Dictyna species, Lathys Simon, 1884 (not fewer than 2 species), Nigma Lehtinen, 1967 (1-2 species); Linyphiidae - several dozens species; Philodromidae - Thanatus C.L. Koch, 1837 (2-4 species), Tibellus Simon, 1875 (1-2 species) and more Philodromus species; Pholcidae - Spermophora senoculta (Duges, 1836); Salticidae - Dendryphantes C.L. Koch, 1837, Pellenes Simon, 1876, Philaeus Thorell, 1869, Synageles Simon, 1876, more Heliophanus, Salticus and Sitticus species; Tetrgnathidae - Pachygnatha listeri Sundevall, 1830, Tetragnatha extensa (Linnaeus, 1757), T. pinicola L. Koch, 1870; Theridiidae Coscinida tibialis Simon, 1895, Lasaeola Simon, 1881, Pholcomma gibbium (Westring, 1851); Zodariidae more Zodarion species.

ACKNOWLEDGEMENTS. M.K. provides many thanks to R.S. Dbar (Sukhum, Abkhazia) for a lot of logistic help in expeditions in Abkhazia in 2008-2009, and N.N. Yunakov (St. Petersburg, Russia), A.A. Khaustov (Yalta, Ukraine), O.S. Bezman-Moseyko and E.G. Sergeeva (both - St-Petersburg, Russia) for the help during the expedition in 2008.

Authors sincerely thank T. Blick (Hummeltal, Germany), K.G. Mikhailov (Moscow, Russia) and S. Otto (Wuerzburg, Germany) and other colleagues for their great help in the search of the relevant references. We are deeply obliged to Yu.G. Arzanov, P.P. Ivliev and E.A. Khachikov (all from Rostov-on-Don, Russia), A.A. Khaustov, A. Shavlokhov (Gagra, Abkhazia) and N.N. Yunakov for providing specimens collected in Abkhazia. We sincerely thank P.E. Gol'din (Simferopol) who improved the English of the earlier draft and S. Otto for some important remarks and corrections. English of the final draft was checked by R. Leech (Edmonton, Canada). 
Table 1. List of families found in Abkhazia and their species richness. Таблица 1. Список семейств, выявленных в Абхазии, и их видовое богатство.

\begin{tabular}{|c|c|c|c|c|c|}
\hline & Families & $\begin{array}{l}\text { Literature } \\
\text { records }\end{array}$ & $\begin{array}{l}\text { Number of identified } \\
\text { species by present data }\end{array}$ & $\begin{array}{l}\text { Total } \\
\text { number }\end{array}$ & $\begin{array}{l}\text { Number of unidentified } \\
\text { species in collections }\end{array}$ \\
\hline 1 & Agelenidae & 4 & 5 & 7 & $4-5$ \\
\hline 2 & Amaurobiidae & 1 & 1 & 1 & 0 \\
\hline 3 & Anyphaenidae & 1 & 1 & 1 & 0 \\
\hline 4 & Araneidae & 13 & 12 & 22 & $2-3$ \\
\hline 5 & Atypidae & 0 & 1 & 1 & 1 \\
\hline 6 & Cheiracanthiidae & 2 & 2 & 2 & $2-3$ \\
\hline 7 & Clubionidae & 2 & 5 & 6 & $2-3$ \\
\hline 8 & Coelotidae & 0 & 0 & 0 & 1 \\
\hline 9 & Corinnidae & 1 & 2 & 2 & $1-2$ \\
\hline 10 & Cybaeidae & 1 & 1 & 1 & 0 \\
\hline 11 & Dictynidae & 0 & 2 & 2 & $2-3$ \\
\hline 12 & Dysderidae & 9 & 6 & 11 & $1-2$ \\
\hline 13 & Filistatidae & 0 & 0 & 0 & 1 \\
\hline 14 & Gnaphosidae & 6 & 9 & 13 & $3-4$ \\
\hline 15 & Hahniidae & 0 & $1+1^{*}$ & 2 & $1-2$ \\
\hline 16 & Leptonetidae & 0 & 1 & 1 & 0 \\
\hline 17 & Linyphiidae & 28 & 58 & 64 & $10-15$ \\
\hline 18 & Liocranidae & 0 & 1 & 1 & 0 \\
\hline 19 & Lycosidae & 11 & 26 & 29 & $5-7$ \\
\hline 20 & Mimetidae & 1 & 2 & 3 & 0 \\
\hline 21 & Mysmenidae & 0 & 1 & 1 & 0 \\
\hline 22 & Nemesiidae & 1 & 1 & 1 & 0 \\
\hline 23 & Nesticidae & 4 & $0^{*}$ & 4 & 4 \\
\hline 24 & Oecobiidae & 1 & 1 & 1 & 0 \\
\hline 25 & Oonopidae & 1 & 0 & 1 & 0 \\
\hline 26 & Oxyopidae & 1 & 1 & 1 & 0 \\
\hline 27 & Philodromidae & 0 & 3 & 3 & $1-2$ \\
\hline 28 & Pholcidae & 2 & 2 & 2 & 1 \\
\hline 29 & Pisauridae & 2 & 1 & 2 & 0 \\
\hline 30 & Salticidae & 14 & 20 & 24 & $4-5$ \\
\hline 31 & Scytodidae & 1 & 1 & 1 & 0 \\
\hline 32 & Segestriidae & 3 & 2 & 3 & 0 \\
\hline 33 & Sparassidae & 1 & 1 & 1 & 0 \\
\hline 34 & Tetragnathidae & 7 & 5 & 8 & $2-3$ \\
\hline 35 & Theridiidae & 14 & 26 & 31 & $7-8$ \\
\hline 36 & Theridiosomatidae & 0 & 1 & 1 & 0 \\
\hline 37 & Thomisidae & 14 & 14 & 20 & $5-7$ \\
\hline 38 & Uloboridae & 3 & 1 & 3 & 0 \\
\hline 39 & Zodariidae & 1 & 1 & 1 & 1 \\
\hline 40 & Zoridae & 0 & 2 & 2 & $1-2$ \\
\hline 41 & Zoropsidae & 1 & 1 & 1 & 0 \\
\hline 42 & Zygiellidae & 1 & 2 & 2 & 0 \\
\hline & Total & 152 & 228 & 283 & $60-80$ \\
\hline
\end{tabular}

"We collected some specimens of this family, but their identification is problematic before revision.

This work of M.K. was supported in part by the Karadag Nature Reserve and Russian Foundation for Basic Research (grants \#\# 09-04-90900 — моб._снг_ст. and 09-04-01365_a).

\section{References}

Blandin P. 1976. Etudes sur les Pisauridae africaines VI. Definition des genres Pisaura Simon, 1885, Pisaurellus Roewer, 1961, Afropisaura n. gen. et mise au point sur les espèces des genres Afropisaura and Pisaurellus (Araneae Pisauridae Pisaurinae) // Revue zool. afr. T.90. Fasc.4. P.917-939.

Brignoli P.M. 1978. Ragni di Turchia V. Specie nuove o interessanti, cavernicole ed epigee, di verie famiglie (Araneae) // Revue suisse Zool. T.85. Fasc.3. P.461-541.

Brignoli P.M. 1984. Zur Problematik der mediterranen PisauraArten (Arachnida, Araneae, Pisauridae) // Zool. Anz. Bd.213. Nr.1/2. S.33-43.

Buchar J. 1966. Beitrag zur Kenntnis der Paläarktischen PirataArten (Araneae, Lycosidae) // Acta societatis zoologicae Bohemoslovacae. Svazek 30. Cislo 3. S.210-218. 
Table 2. Distribution range composition of identified species in Abkhazia. Таблица 2. Ареалогический состав пауков в Абхазии (только идентифицированные виды).

\begin{tabular}{|l|c|c|}
\hline Ranges & $\begin{array}{l}\text { Number of } \\
\text { identified species }\end{array}$ & $\%$ \\
\hline $\begin{array}{l}\text { Palaearctic (Trans-Palaearctic; West and Central Palaearctic; West Palaearctic; West } \\
\text { Palaearctic and Kopetdagh Mt.) }\end{array}$ & 162 & $\sim 57.3$ \\
\hline Caucasus (West Caucasus; Caucasus Major; Caucasus Major and Armenian Upland) & 33 & $\sim 12$ \\
\hline Holarctic & 32 & $\sim 11$ \\
\hline $\begin{array}{l}\text { Ancient Mediterranean (Mediterranean-Caucasus ranges; ranges from Balkans to Kopetdagh } \\
\text { and/or Middle Asia; East Europe-Balkans-Crimea-Caucasus; Mediterranean-Caucasus; } \\
\text { Balkans-Turkey-Caucasus; Balkans-Caucasus) }\end{array}$ & 24 & $\sim 8.5$ \\
\hline Cosmopolitan & & \\
\hline Crimea-Caucasus & 9 & $\sim 3$ \\
\hline Disjunctive range: Europe and Caucasus - or Caucasus - Far East & 7 & $\sim 2.5$ \\
\hline Anatolia-Caucasus & 6 & $\sim 2.1$ \\
\hline Crimea-Anatolia-Caucasus & 3 & $\sim 1.1$ \\
\hline Anatolia-Caucasus-Middle Asian Mts.-Iran & 2 & $\sim 0.7$ \\
\hline Iran-Caucasus & 2 & $\sim 0.7$ \\
\hline Crimea-Caucasus-Iran-Middle Asian Mts. & 2 & $\sim 0.7$ \\
\hline Total & 1 & $\sim 0.4$ \\
\hline
\end{tabular}

Charitonov D.E. 1932. Katalog der Russischen spinnen. Leningrad: Academie des sciences de L'URSS. Beilage zum “Annuaire du Musée Zoologique". Vol.32. 206 S [in German and Russian].

Charitonov D.E. 1936. [An addition to the catalogue of Russian spiders] // Uchenie zapiski Permskogo universiteta. T.2. No.1. P.167-225 [in Russian].

Charitonov D.E. 1939. [On the cave spiders of Abkhazia] // Materialy $\mathrm{k}$ faune Abkhazii. Tbilisi: Gruzinskyi filial AN SSSR. P.197-211 [in Russian].

Charitonov D.E. 1941. New materials of the Arachnoidea of Abkhasia caves // Trudy Zoologicheskogo instituta AN GSSR. T.4. P.165-175 [in Russian].

Charitonov D.E. 1947. Biospeologica sovietica. VIII. Spiders and harvestspiders from the caves of the Black Sea Coast of the Caucasus // Bulleten' Moskovskogo Obshchestva Ispytatelei Prirody. T.52. No.2. P.15-28 [in Russian]

Dunin P.M. 1986. The spider family Segestriidae in the USSR fauna (Arachnida: Araneae: Haplogynae) // Senckenbergiana biol. Bd.66. H.4/6. P.327-332.

Dunin P.M. 1992a. [New spider species in the genus Harpactea in the Caucasus fauna (Aranei, Haplogynae, Dysderidae)] // Zool. zhurnal. T.71. No.1. P.58-62 [in Russian].

Dunin P.M. 1992b. [The spider family Dysderidae of the Caucasian fauna (Arachnida, Aranei, Haplogynae)] // Arthropoda Selecta. Vol.1. No.3. P.35-76 [in Russian].

Dzhanashvili R.A. 1965. [Some data on the cavernicolous fauna of Georgia]// Sbornik speleologicheskoy komissii "Peshchery Gruzii”". Akademia nauk Gruzinskoy SSR. T.3. P.21-24 [in Russian].

Esyunin S.L., Efimik V.E. 1997. Remarks on the Ural spider fauna, 6. New data on the taxonomy and faunistics of gnaphosid spiders of the South Urals (Arachnida, Aranei, Gnaphosidae) // Arthropoda Selecta. Vol.5. No.3-4. P.105-111.

Esyunin S.L., Farzalieva G.Sh. 2002. Redescription of Tegenaria taurica Charitonov, 1947 (Aranei: Agelenidae) // Arthropoda Selecta. Vol.10. No.3. P.261-263.

Gorodkov K.B. 1984. [Types of ranges of insects inhabiting tundra and taiga zones of European part of USSR] // Insect ranges of the European part of USSR, Maps 179-221. Leningrad: Nauka Press. P.3-20 [in Russian].

Helsdingen van P. 2010. Fauna Europaea: Araneae. Fauna Europaea version $2.2,<$ http://www.faunaeur.org $>$.

Koch L. 1878. Kaukasische Arachnoiden // Schneider O. (ed.) Naturwissenschaftliche Beiträge zur Kenntniss der Kaukasuslander. Dresden: Verlag der Burdach'schen Hofbuchhandlung. S.36-71.
Kovblyuk M.M. 2004a. [Redescription and synonymy of Tegenaria lapicidinarum (Aranei, Agelenidae)] // Vestnik zoologii. Vol.38. No.3. P.43-51 [in Russian].

Kovblyuk M.M. 2004b. A survey of spider species with CrimeaCaucasian disjunct ranges (Arachnida, Araneae) // Samu F. \& Szinetar Cs. (eds.). European Arachnology 2002 (Proceedings of the 20th European Colloquium of Arachnology, Szombathely 22-26 July 2002). Plant Protection Institute \& Berzsenyi College, Budapest. P.251-258.

Kovblyuk M.M. 2004c. [Catalogue of the spiders (Arachnida, Aranei) of the Crimea] // Voprosy razvitiya Kryma. Vyp.15. Problemy inventarizatsii krymskoi bioty. Simferopol: Tavriya-Plus. P.211-262 [in Russian].

Kovblyuk M.M. 2005. The spider genus Gnaphosa Latreille, 1804 in the Crimea (Aranei: Gnaphosidae) // Arthropoda Selecta. Vol.14. No.2. P.133-152.

Kovblyuk M.M., Nadolny A.A. 2009. The spider genus Trachelas L.Koch, 1872 in Crimea and Caucasus with the description of Paratrachelas gen.n. (Aranei: Corinnidae) // Arthropoda Selecta. Vol.18. No.1-2. P.35-46.

Kovblyuk M.M., Ponomarev A.V. 2008. [New and interesting spiders (Aranei: Agelenidae, Corinnidae, Gnaphosidae, Nemesiidae, Thomisidae) from the West Caucasus] // Caucasian entomological bull. Vol.4. No.2. P.143-154 [in Russian]

Kovblyuk M.M., Ponomarev A.V., Dvadnenko K.V. 2010. Redescription of Cybaeus abchasicus Charitonov, 1947, with the first description of the male (Aranei: Cybaeidae) // Arthropoda Selecta. Vol.19. No.4. P.221-225.

Kovblyuk M.M., Prokopenko E.V., Nadolny A.A. 2008. [Spider family Dysderidae of the Ukraine (Arachnida, Aranei)] // Euroasian Entomological Journal. Vol.7. No.4. P.287-306 [in Russian]

Kryzhanovsky O.L. 2002. [Composition and distribution of entomological faunas in the World]. Moscow: KMK Scientific Press Ltd. 237 p. [in Russian].

Logunov D.V. 2006. Notes on Xysticus kempeleni Thorell, 1872 and two closely related spider species (Araneae, Thomisidae) // Acta Arachnologica. Vol.55. No.1. P.59-66.

Logunov D.V., Guseinov E.F. 2002. Faunistic review of the jumping spiders of Azerbaijan (Aranei: Salticidae), with additional faunistic records from neighbouring Caucasian countries // Arthropoda Selecta. Vol.10. No.3. P.243-260.

Logunov D.V., Marusik Yu.M. 1990. [Spiders of the genus Argyrodes (Aranei, Theridiidae) of the USSR] // Zoologicheskiy zhurnal. T.69. Vyp.2. P.133-136 [in Russian].

Logunov D.V., Marusik Yu.M. 1992. [Spiders of the genus Phoroncidia (Aranei, Theridiidae) of the USSR] // Fauna i ecologiya 
paukov, skorpionov i lozhnoskorpionov SSSR. Trudy Zoologicheskogo instituta AN SSSR. T.226 (1990). P.91-97 [in Russian].

Logunov D.V., Marusik Yu.M. 2000. Catalogue of the jumping spiders of northern Asia (Arachnida, Araneae, Salticidae). Moscow: KMK Scientific Press Ltd. 299 p.

Logunov D.V. 2006. Notes on Xysticus kempeleni Thorell, 1872 and two closely related spider species (Araneae, Thomisidae) // Acta Arachnologica. Vol.55. No.1. P.59-66.

Lyovushkin S.I. 1966. [Fauna of the two caves of Garga District (after collections of expedition of speleological comission of AN Georgian SSR in August 1962)] // Peshchery Gruzii (Speleologicheskyi sbornik). Vol.4. P.116-120 [in Russian].

Marusik Yu.M. 1989. [New data on the fauna and synonymy of spiders (Arachnida: Aranei) from the USSR] // Fauna i ekologia paukov i skorpionov. Arakhnologicheskiy sbornik. Moscow: Nauka. P.39-52 [in Russian].

Marusik Yu.M. 2005. A new family and interesting new records of spiders (Aranei) from the European part of Russia // Arthropoda Selecta. Vol.14. No.1. P.89-91.

Marusik Yu.M., Gnelitsa V.A. 2009. Description of a new genus of spiders from the eastern Mediterranean and the most armored erigonid species from the western Caucasus (Aranei: Linyphiidae: Erigononae) // Arthropoda Selecta. Vol.18. No.1-2. P.5768.

Marusik Yu.M., Guseinov E.F. 2003. Spiders (Arachnida, Aranei) of Azerbaijan. 1. New family and genus records // Arthropoda Selecta. Vol.12. No.1. P.29-46.

Marusik Yu.M., Guseinov E.F., Koponen S., Yoshida H. 2004. A new case of Caucasus-Far East disjunctive range in spider (Araneae) // Acta Arachnologica. Vol.53. No.2. P.125-129.

Marusik Yu.M., Mikhailov K.G., Guseinov E.F. 2006. Advances in study of biodiversity of Caucasian spiders (Araneae) // Deltshev C., Stoev P. (eds.). European Arachnology 2005. Acta Zoologica Bulgarica. Suppl. No.1. P.259-268.

Marusik Yu.M., Kovblyuk M.M. 2004. New and interesting cribellate spiders from Abkhazia (Aranei: Amaurobiidae, Zoropsidae) // Arthropoda Selecta. Vol.13. No.1-2. P.55-61.

Marusik Yu.M., Kunt K.B., Danisman T. 2009. Spiders (Aranei) new to the fauna of Turkey. 2. New species records of Theridiidae // Arthropoda Selecta. Vol.18. No.1-2. P.69-75.

Marusik Yu.M., Kovblyuk M.M. 2010. The spider genus Trachelas L. Koch, 1872 (Aranei: Corinnidae) in Russia // Arthropoda Selecta. Vol.19. No.1. P.21-27.

Marusik Yu.M., Logunov D.V., Koponen S. 2000. Spiders of Tuva, South Siberia. Magadan: IBPN FEB RAS. 252 p.

Mcheidze T.S. 1964. [Spiders (Araneina)] // Zhivotnyi Mir Gruzii. Tbilisi: AN Gruz. SSR Publ. T.2. P.48-116 [in Georgian].

Mcheidze T.S. 1997. [Spiders of Georgia (taxonomy, ecology, zoogeographical review)]. Tbilisi: Tbilisi University. 390 p. [in Georgian]

Mcheidze T.S., Utochkin A.S. 1971. [New species of spiders of the genus Xysticus C.L. Koch from Georgia] // Bulletin of the Academy of sciences of the Georgian SSR. T.64. No.1. P. 209-212 [in Russian].

Mikhailov K.G. 1990. The spider genus Clubiona Latreille, 1804 in the Caucasus, USSR // Senckenbergiana biol. Vol.70. No.4/ 6. P. 299-322.

Mikhailov K.G. 1997. Catalogue of the spiders of the territories of the former Soviet Union (Arachnida, Aranei). Moscow: Zoological Museum of the Moscow State University. $416 \mathrm{p}$.

Nadolny A.A., Kovblyuk M.M. 2006. Carpathonesticus borutzkyi, the first record of Nesticidae from the Crimea (Aranei: Nesticidae) // Arthropoda Selecta. Vol.15. No.4 P.291-294.

Nishikawa Y. 2009. A new genus and 44 new species of the family Coelotidae (Arachnida, Araneae) from Japan // Ono H. (ed.). The spiders of Japan with keys to the families and genera and illustrations of species. Kanagawa: Tokai Univ. Press. P.51-70 [in Japanese].

Otto S., Dietzold S. 2006. Caucasian Spiders. A faunistic database on the spiders of the Caucasus. Version 1.3. http://caucasusspiders.info
Ovtsharenko V.I. 1979. [Spiders of the families Gnaphosidae, Thomisidae, Lycosidae (Aranei) of the Caucasus Major] // Fauna i ekologia paukoobraznykh. Trudy Zoologicheskogo instituta AN SSSR. T.85. P.39-53 [in Russian].

Ovtsharenko V.I. 1982. [A systematic list of the spider family Gnaphosidae (Aranei) of the European part of the USSR and the Caucasus] // Entomologicheskoe obozrenie. T.61. No.4. P.830-844 [in Russian].

Ovtsharenko V.I., Platnick N.I., Song D.X. 1992. A review of the North Asian spiders of the genus Gnaphosa (Araneae, Gnaphosidae) // Bull. Amer. Mus. Natur. Hist. No.212. P.1-88.

Pichka V.E. 1965. [On the spider fauna of the caves in the West Transcaucasia] // Zool. zhurnal. T.44. No.8. P.1190-1196 [in Russian].

Platnick N.I. 2010. The world spider catalog, version 10.5. American Museum of Natural History, online at $<\mathrm{http}$ ://research.amnh. org/entomology/spiders/catalog/index.html>.

Ponomarev A.V. 2009. [New species and finds of spiders (Aranei) from the south of Russia and Western Kazakhstan] // Caucasian Entomological Bulletin. Vol.5. No.2. P.143-146 [in Russian].

Ponomarev A.V., Alieva S.V. 2010. [The new data on spiders (Aranei) fauna of Dagestan] // Vestnik Permskogo Universiteta. Biologia. Vyp.3. P.12-16 [in Russian].

Ponomarev A.V., Chumachenko Yu.A. 2007. [Arachnida in Ground Mesofauna of Yewbox Grove of the Caucasian Biospheric Reserve] // Lebedeva N.V. (ed.). Bioraznoobrazie I transformatsiya gornykh ekosistem Kavkaza. Trudy Yuzhnogo nauchnogo tsentra RAN. T.3. Rostov-on-Don: YuNTs RAN. P.151163 [in Russian].

Prószyński J. 1979. Systematic studies on East Palaearctic Salticidae III. Remarks on Salticidae of the USSR // Annales Zoologici Warszawa. T.34. Nr.11. P.299-369.

Reimoser E. 1930. Eine neue Nesticus-Art aus dem Kaukasus // Zoologischer Anzeiger. Bd.88. Nr.5/6. S.158-159.

Schmidt P. 1895. Beitrag zur Kenntniss der Laufspinnen (Araneae Citigradae Thor.) Russlands // Zoologische Jahrbucher. Abtheilung für Systematik, Geographie und Biologie der Thiere. Bd.8. Nr.4. S.439-484.

Simon E. 1898. Histoire naturelle des Araignées. T.2. Paris. P.193380 .

Simon E. 1899. Araneae Caucasicae // G.I. Radde. Kollektsii Kavkazskogo Muzeya (Museum Caucasicum). T.1. Zoologiya. Tiflis: Tipographiya Kancelyarii Glavnonachalstvuyushchego grazhdanskoyu chastiyu na Kavkaze. P.478-480.

Spassky S.A. 1932. Araneae species novae II // Bull. Mus. Hist. Natur. Paris. Ser.2. T.4. No.2. P.972-979.

Spassky S.A. 1934. Araneae palaearcticae novae. Fam. Pholcidae I // Bull. Mus. Hist. Natur. Paris. Serie 2. Tome 6. No.4. P.361372.

Spassky S.A. 1937. [Some facts on the spider fauna of the Black Sea coast] // Sbornik nauchno-issledovatelskikh rabot AzovoChernomorskogo selsko-khozyaystvennogo instituta. No.5. P.131-138 [in Russian].

Tanasevitch A.V. 1986. Two new Troglohyphantes from the Caucasus // Spixiana. Vol.9. No.3. P.239-243.

Tanasevitch A.V. 1987. The liniphiid spiders of the Caucasus, USSR (Arachnida: Araneae: Linyphiidae) // Senckenbergiana biol. Vol.67. No.4/6. P.297-383.

Tanasevitch A.V. 1990. [Spiders of the family Linyphiidae of the Caucasian fauna (Arachnida, Aranei)] // Striganova B.R. (ed.). Fauna nazemnikh bespozvonochnykh Kavkaza. Moscow: Nauka. P.5-114, 235 [in Russian].

Tanasevitch A.V. 2009. The linyphiid spiders of Iran (Arachnida, Araneae, Linyphiidae) // Revue suisse de Zoologie. T.116. Fasc.3-4. P.379-420.

Verzhbitsky E. 1902. [On the spiders of the Caucasian Province] // Zapisky Kievskago obshchestva estestvoispytateley. T.17. Vyp.2. P.461-504 [in Russian].

Wagner W. 1896. [Biological notes on the fauna of the eastern coast of the Black Sea (a preliminary report)] // Trudy imperatorskogo S.-Peterburgskago obshchestva yestestvoispytatelei. T.27. Vyp.1. No.7-8. P.267-282 [in Russian]. 
Zyuzin A.A., Logunov D.V. 2000. New and little-known species of Lycosidae from Azerbaijan, the Caucasus (Araneae, Lycosidae) // Bull. Br. arachnol. Soc. Vol.11. Pt.8. P.305-319.

Zyuzin A.A., Ovtsharenko V.I. 1979. [Taxonomic remarks on Pardosa incerta Nosek (Aranei, Lycosidae), spider species new to the USSR fauna] // Balashov Yu.S. (ed.). Fauna i ekologia paukoobraznykh. Trudy Zoologicheskogo instituta AN SSSR. T.85. P.60-64 [in Russian].

Responsible editor K.G. Mikhailov 